\title{
THE GEOMETRY OF HIDA FAMILIES I: $\Lambda$-ADIC DE RHAM COHOMOLOGY
}

\author{
BRYDEN CAIS
}

To Masami Ohta

\begin{abstract}
We construct the $\Lambda$-adic de Rham analogue of Hida's ordinary $\Lambda$-adic étale cohomology and of Ohta's $\Lambda$-adic Hodge cohomology, and by exploiting the geometry of integral models of modular curves over the cyclotomic extension of $\mathbf{Q}_{p}$, we give a purely geometric proof of the expected finiteness, control, and $\Lambda$-adic duality theorems. Following Ohta, we then prove that our $\Lambda$-adic module of differentials is canonically isomorphic to the space of ordinary $\Lambda$-adic cuspforms. In the sequel [Cais3] to this paper, we construct the crystalline counterpart to Hida's ordinary $\Lambda$-adic étale cohomology, and employ integral $p$-adic Hodge theory to prove $\Lambda$-adic comparison isomorphisms between all of these cohomologies. As applications of our work in this paper and [Cais3], we will be able to provide a "cohomological" construction of the family of $(\varphi, \Gamma)$-modules attached to Hida's ordinary $\Lambda$-adic étale cohomology by [Dee], as well as a new and purely geometric proof of Hida's finiteness and control theorems. We are also able to prove refinements of the main theorems in [MW3] and [Ohta1].
\end{abstract}

\section{INTRODUCTION}

1.1. Motivation. In his landmark papers [Hida1] and [Hida2], Hida proved that the $p$-adic Galois representations attached to ordinary cuspidal Hecke eigenforms by Deligne ([Deligne1], [Carayol]) interpolate $p$-adic analytically in the weight variable to a family of $p$-adic representations whose specializations to integer weights $k \geq 2$ recover the "classical" Galois representations attached to weight $k$ cuspidal eigenforms. Hida's work paved the way for a revolution - from the pioneering work of Mazur on Galois deformations to Coleman's construction of $p$-adic families of finite slope overconvergent modular forms - and began a trajectory of thought whose fruits include some of the most spectacular achievements in modern number theory.

Hida's proof is constructive and has at its heart the étale cohomology of the tower of modular curves $\left\{X_{1}\left(N p^{r}\right)\right\}_{r}$ over $\mathbf{Q}$. More precisely, Hida considers the projective limit $H_{\text {ét }}^{1}:=\lim _{r} H_{\text {ét }}^{1}\left(X_{1}\left(N p^{r}\right)_{\overline{\mathbf{Q}}}, \mathbf{Z}_{p}\right)$ (taken with respect to the trace mappings), which is naturally a module for the "big" $p$-adic Hecke algebra $\mathfrak{H}^{*}:=\lim _{\leftarrow} \mathfrak{H}_{r}^{*}$, which is itself an algebra over the completed group ring $\Lambda:=\mathbf{Z}_{p} \llbracket 1+p \mathbf{Z}_{p} \rrbracket \simeq \mathbf{Z}_{p} \llbracket T \rrbracket$ via the diamond operators. Using the idempotent $e^{*} \in \mathfrak{H}^{*}$ attached to the (adjoint) Atkin operator $U_{p}^{*}$ to project to the part of $H_{\text {ét }}^{1}$ where $U_{p}^{*}$ acts invertibly, Hida proves in [Hida1, Theorem 3.1] (via the comparison isomorphism between étale and topological cohomology and explicit calculations in group cohomology) that $e^{*} H_{\text {ét }}^{1}$ is finite and free as a module over $\Lambda$, and that the resulting Galois representation

$$
\rho: \mathscr{G}_{\mathbf{Q}} \longrightarrow \operatorname{Aut}_{\Lambda}\left(e^{*} H_{\text {ét }}^{1}\right) \simeq \operatorname{GL}_{m}\left(\mathbf{Z}_{p} \llbracket T \rrbracket\right)
$$

Date: October 16, 2017.

2010 Mathematics Subject Classification. Primary: 11F33 Secondary: 11F67, 11G18, 11R23.

Key words and phrases. Hida families, integral p-adic Hodge theory, de Rham cohomology, crystalline cohomology.

During the writing of this paper, the author was partially supported by an NSA Young Investigator grant (H9823012-1-0238) and an NSF RTG (DMS-0838218). 
$p$-adically interpolates the representations attached to ordinary cuspidal eigenforms.

By analyzing the geometry of the tower of modular curves, Mazur and Wiles [MW3] were able to relate the inertial invariants of the local (at $p$ ) representation $\rho_{p}$ to the étale cohomology of the Igusa tower studied in [MW1], and in so doing proved ${ }^{1}$ that the ordinary filtration of the Galois representations attached to ordinary cuspidal eigenforms can be $p$-adically interpolated: both the inertial invariants and covariants are free of the same finite rank over $\Lambda$ and specialize to the corresponding subquotients in integral weights $k \geq 2$. As an application, they provided examples of cuspforms $f$ and primes $p$ for which the specialization of the associated Hida family of Galois representations to weight $k=1$ is not Hodge-Tate, and so does not arise from a weight one cuspform via the construction of Deligne-Serre [DS]. Shortly thereafter, Tilouine [Tilouine] clarified the geometric underpinnings of [Hida1] and [MW3], and removed most of the restrictions on the p-component of the nebentypus of $f$. Central to both [MW3] and [Tilouine] is a careful study of the tower of $p$-divisible groups attached to the "good quotient" modular abelian varieties introduced in [MW2].

With the advent of integral $p$-adic Hodge theory, and in view of the prominent role it has played in furthering the trajectory initiated by Hida's work, it is natural to ask if one can construct HodgeTate, de Rham and crystalline analogues of $e^{*} H_{\text {ét }}^{1}$, and if so, to what extent the integral comparison isomorphsms of $p$-adic Hodge theory can be made to work in $\Lambda$-adic families. In [Ohta1], Ohta has addressed this question in the case of Hodge cohomology. Using the invariant differentials on the tower of $p$-divisible groups studied in [MW3] and [Tilouine], Ohta constructs a $\Lambda \widehat{\otimes} \mathbf{z}_{p} \mathbf{Z}_{p}\left[\mu_{p}\right.$ ]-module from which, via an integral version of the Hodge-Tate comparison isomorphism [Tate1] for ordinary $p$ divisible groups, he is able to recover the semisimplification of the "semilinear representation" $\rho_{p} \widehat{\otimes} \mathscr{O}_{\mathbf{C}_{p}}$, where $\mathbf{C}_{p}$ is, as usual, the $p$-adic completion of an algebraic closure of $\mathbf{Q}_{p}$. Using Hida's results, Ohta proves that his Hodge cohomology analogue of $e^{*} H_{\text {ét }}^{1}$ is free of finite rank over $\Lambda \widehat{\otimes} \mathbf{z}_{p} \mathbf{Z}_{p}\left[\mu_{p^{\infty}}\right]$ and specializes to finite level exactly as one expects. As applications of his theory, Ohta provides a construction of two-variable $p$-adic $L$-functions attached to families of ordinary cuspforms differing from that of Kitagawa [Kitagawa], and, in a subsequent paper [Ohta2], provides a new and streamlined proof of the theorem of Mazur-Wiles [MW2] (Iwasawa's Main Conjecture for Q; see also [Wiles2]). We remark that Ohta's $\Lambda$-adic Hodge-Tate isomorphism is a crucial ingredient in the forthcoming partial proof of Sharifi's conjectures [Sharifi2], [Sharifi1] due to Fukaya and Kato [FK].

1.2. Results. In this paper, we construct the de Rham counterpart to Hida's ordinary $\Lambda$-adic étale cohomology and Ohta's $\Lambda$-adic Hodge cohomology, and we prove the expected control and finiteness theorems via a purely geometric argument involving a careful study of the geometry of certain KatzMazur integral models of modular curves and a classical result of Nakajima [Nakajima].

In the sequel [Cais3] to this paper, we will use crystalline Dieudonné theory to provide a cohomoligcal construction of the $\Lambda$-adic family of $(\varphi, \Gamma)$-modules attached to $e^{*} H_{\text {ét }}^{1}$ by Dee [Dee], and will establish a suitable $\Lambda$-adic version of every integral comparison isomorphism one could hope for. In particular, we will be able to recover the entire family of $p$-adic Galois representations $\rho_{p}$ (and not just its semisimplification) from our $\Lambda$-adic crystalline cohomology. As an application of our theory and the results of this paper, we will in [Cais3] give a new and purely geometric proof of Hida's freeness and control theorems for $e^{*} H_{\text {ét }}^{1}$. As our methods are geometric, we expect that they can be adapted to the setting of other Shimura curves once one has a sufficiently sophisticated theory of integral models.

In order to survey our main results more precisely, we introduce some notation. Throughout, we fix a prime $p>2$ and a positive integer $N>3$ with $p \nmid N$. Fix an algebraic closure $\overline{\mathbf{Q}}_{p}$ of $\mathbf{Q}_{p}$

\footnotetext{
${ }^{1}$ Mazur and Wiles treat only the case of tame level $N=1$.
} 
as well as a $p$-power compatible sequence $\left\{\varepsilon^{(r)}\right\}_{r \geq 0}$ of primitive $p^{r}$-th roots of unity in $\overline{\mathbf{Q}}_{p}$. We set $K_{r}:=\mathbf{Q}_{p}\left(\mu_{p^{r}}\right)$ and $K_{r}^{\prime}:=K_{r}\left(\mu_{N}\right)$, and we write $R_{r}$ and $R_{r}^{\prime}$ for the rings of integers in $K_{r}$ and $K_{r}^{\prime}$, respectively, and put $R_{\infty}:=\cup_{r \geq 1} R_{r}$ and $R_{\infty}^{\prime}:=\cup_{r \geq 1} R_{r}^{\prime}$, etc. Denote by $\mathscr{G}_{\mathbf{Q}_{p}}:=\operatorname{Gal}\left(\overline{\mathbf{Q}}_{p} / \mathbf{Q}_{p}\right)$ the absolute Galois group and by $\mathscr{H}$ the kernel of the $p$-adic cyclotomic character $\chi: \mathscr{G}_{\mathbf{Q}_{p}} \rightarrow \mathbf{Z}_{p}^{\times}$. We write $\Gamma:=\mathscr{G}_{\mathbf{Q}_{p}} / \mathscr{H} \simeq \operatorname{Gal}\left(K_{\infty} / K_{0}\right)$ for the quotient and, using that $K_{0}^{\prime} / \mathbf{Q}_{p}$ is unramified, we canonically identify $\Gamma$ with $\operatorname{Gal}\left(K_{\infty}^{\prime} / K_{0}^{\prime}\right)$. We put $\Delta:=\mathbf{Z}_{p}^{\times}$, and for $r \geq 1$ set $\Delta_{r}:=1+p^{r} \mathbf{Z}_{p}$. For any ring $A$, let $\Lambda_{A, r}:=A\left[\Delta_{1} / \Delta_{r}\right]$ be the group ring on $\Delta_{1} / \Delta_{r}$ over $A$ and write $\Lambda_{A}:=\lim _{r} \Lambda_{A, r}$ for the completed group ring on $\Delta_{1}$ over $A$; if $\varphi$ is an endomorphism of $A$, we again write $\varphi$ for the induced endomorphisms of $\Lambda_{A, r}$ and $\Lambda_{A}$ that act as the identity on $\Delta_{1}$. We will denote by $\langle u\rangle^{*}$ the adjoint diamond operator attached to $u \in \Delta$, and give $\mathfrak{H}_{r}^{*}$ the structure of a $\Lambda$-module via the map $\langle\cdot\rangle^{*}: \Delta_{1} \hookrightarrow \mathfrak{H}^{*}$. Finally, we denote by $X_{r}:=X_{1}\left(N p^{r}\right)$ the canonical model over $\mathbf{Q}$ with rational cusp at $i \infty$ of the modular curve arising as the quotient of the extended upper-halfplane by the congruence subgroup $\Gamma_{1}\left(N p^{r}\right)$, and we write $J_{r}:=J_{1}\left(N p^{r}\right)$ for its Jacobian.

The goal of this paper is to construct a de Rham analogue of Hida's $e^{*} H_{\text {ét }}^{1}$. A naïve idea would be to mimic Hida's construction, using the (relative) de Rham cohomology of $\mathbf{Z}_{p}$-integral models of the modular curves $X_{r}$ in place of $p$-adic étale cohomology. However, this approach fails due to the fact that $X_{r}$ has bad reduction at $p$, so the relative de Rham cohomology of integral models does not provide good $\mathbf{Z}_{p}$-lattices in the de Rham cohomology of $X_{r}$ over $\mathbf{Q}_{p}$. To address this problem, we use the canonical integral structures in de Rham cohomology studied in [Cais1] and the canonical integral

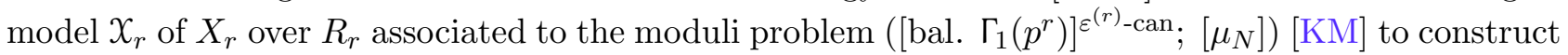
well-behaved integral "de Rham cohomology" for the tower of modular curves. For each $r$, we obtain a short exact sequence of free $R_{r}$-modules with semilinear $\Gamma$-action and commuting $\mathfrak{H}_{r}^{*}$-action

$$
0 \longrightarrow H^{0}\left(X_{r}, \omega_{X_{r} / R_{r}}\right) \longrightarrow H^{1}\left(X_{r} / R_{r}\right) \longrightarrow H^{1}\left(X_{r}, \mathscr{O}_{X_{r}}\right) \longrightarrow 0
$$

which is functorial in finite $K_{r}$-morphisms of the generic fiber $X_{r}$, and whose scalar extension to $K_{r}$ recovers the Hodge filtration of $H_{\mathrm{dR}}^{1}\left(X_{r} / K_{r}\right)$. Extending scalars to $R_{\infty}$ and taking projective limits we obtain an exact sequence of $\Lambda_{R_{\infty}}$-modules with semilinear $\Gamma$-action and commuting linear $\mathfrak{H}^{*}$-action

$$
0 \longrightarrow H^{0}(\omega) \longrightarrow H_{\mathrm{dR}}^{1} \longrightarrow H^{1}(\mathscr{O}) \text {. }
$$

Our main result (see Theorem 3.2.3) is that the ordinary part of (1.2.2) is the correct de Rham analogue of Hida's ordinary $\Lambda$-adic étale cohomology:

Theorem 1.2.1. There is a canonical short exact sequence of finite free $\Lambda_{R_{\infty}}$-modules with semilinear $\Gamma$-action and commuting linear $\mathfrak{H}^{*}$-action

$$
0 \longrightarrow e^{*} H^{0}(\omega) \longrightarrow e^{*} H_{\mathrm{dR}}^{1} \longrightarrow e^{*} H^{1}(\mathscr{O}) \longrightarrow 0 .
$$

As a $\Lambda_{R_{\infty}}$-module, $e^{*} H_{\mathrm{dR}}^{1}$ is free of rank $2 d$, while each of the flanking terms in (1.2.3) is free of rank $d$, for $d=\sum_{k=3}^{p+1} \operatorname{dim}_{\mathbf{F}_{p}} S_{k}\left(\Gamma_{1}(N) ; \mathbf{F}_{p}\right)^{\text {ord }}$. Applying $\otimes_{\Lambda_{R_{\infty}}} R_{\infty}\left[\Delta_{1} / \Delta_{r}\right]$ to (1.2.3) recovers the ordinary part of the scalar extension of (1.2.1) to $R_{\infty}$.

We then show that the $\Lambda_{R_{\infty}}$-adic Hodge filtration (1.2.3) is very nearly "auto dual". To state our duality result more succintly, for any ring homomorphism $A \rightarrow B$, we will write $(\cdot)_{B}:=(\cdot) \otimes_{A} B$ and $(\cdot)_{B}^{\vee}:=\operatorname{Hom}_{B}\left((\cdot) \otimes_{A} B, B\right)$ for these functors from $A$-modules to $B$-modules. If $G$ is any group of automorphisms of $A$ and $M$ is an $A$-module with a semilinear action of $G$, for any "crossed" 
homomorphism $^{2} \psi: G \rightarrow A^{\times}$we will write $M(\psi)$ for the $A$-module $M$ with "twisted" semilinear $G$-action given by $g \cdot m:=\psi(g) g m$. Our duality theorem is (see Proposition 3.2.4):

Theorem 1.2.2. The natural cup-product auto-duality of (1.2.1) over $R_{r}^{\prime}:=R_{r}\left[\mu_{N}\right]$ induces a canonical $\Lambda_{R_{\infty}^{\prime}}$-linear and $\mathfrak{H}^{*}$-equivariant isomorphism of exact sequences

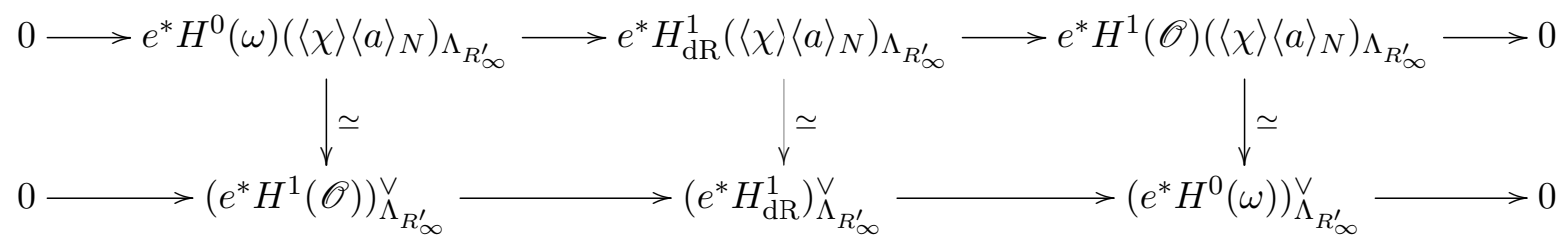

that is compatible with the natural action of $\Gamma \times \operatorname{Gal}\left(K_{0}^{\prime} / K_{0}\right) \simeq \operatorname{Gal}\left(K_{\infty}^{\prime} / K_{0}\right)$ on the bottom row and the twist of the natural action on the top row by the $\mathfrak{H}^{*}$-valued character $\langle\chi\rangle\langle a\rangle_{N}$, where $a(\gamma) \in(\mathbf{Z} / N \mathbf{Z})^{\times}$ is determined for $\gamma \in \operatorname{Gal}\left(K_{0}^{\prime} / K_{0}\right)$ by $\zeta^{a(\gamma)}=\gamma \zeta$ for every $N$-th root of unity $\zeta$.

We moreover prove that, as one would expect, the $\Lambda_{R_{\infty}}$-module $e^{*} H^{0}(\omega)$ is canonically isomorphic to the module $e S\left(N, \Lambda_{R_{\infty}}\right)$ of ordinary $\Lambda_{R_{\infty}}$-adic cusp forms of tame level $N$; see Corollary 3.3.5.

1.3. Overview of the article. We now describe the contents and structure of the article in more detail. Appendix A is devoted to reviewing and extending the theory of [Cais1], which provides the "good integral structures" in the de Rham cohomology of curves that play a central role in our constructions; in particular, the existence and properties of the short exact sequence (1.2.1) are discussed in the generality that we shall need them. As this discussion will be applied to certain integral (Katz-Mazur) models of modular curves (like $X_{r}$ ), we review the construction and relevant features of these models, as well as correspondences on them, in Appendix B. The reader who is content to accept this foundational material on faith can safely skip the discussion in Appendices A-B, and refer to it on a "need to know" basis. As we explain below, a key point (which is the reason that we must work over $R_{r}$ ) is that the regular and proper relative curve $\mathcal{X}_{r}$ over $R_{r}$ has reduced special fiber.

Writing $H\left(X_{r} / R_{r}\right)$ for this short exact sequence (1.2.1), the natural degeneracy mappings on modular curves $X_{r+1} \rightarrow X_{r}$ induce trace mappings

$$
\rho_{*}: e_{r+1}^{*} H\left(X_{r+1} / R_{r+1}\right) \rightarrow e_{r}^{*} H\left(X_{r} / R_{r}\right) \otimes_{R_{r}} R_{r+1}
$$

on ordinary parts, and by definition the sequence (1.2.3) is obtained from these mappings by passing to projective limits. In order to prove theorems 1.2 .1 and 1.2.2, we will reduce to working at finite level and in characteristic $p$ via a general commutative algebra formalism for dealing with such projective limits of "towers" of cohomology that we use throughout this paper and its sequel [Cais3]. This formalism, which is the the subject of $\S 3.1$, reduces the proof of Theorem 1.2.1 to the following two assertions:

(1) The terms in the short exact sequence $e_{r}^{*} H\left(X_{r} / R_{r}\right) \otimes_{R_{r}} \mathbf{F}_{p}$ are free $\mathbf{F}_{p}\left[\Delta_{1} / \Delta_{r}\right]$-modules of ranks $d, 2 d$, and $d$, respectively.

(2) For all $r$, the induced maps $\rho_{*} \otimes 1: e_{r+1}^{*} H\left(\mathcal{X}_{r+1} / R_{r+1}\right) \otimes_{R_{r+1}} \mathbf{F}_{p} \longrightarrow e_{r}^{*} H\left(\mathcal{X}_{r} / R_{r}\right) \otimes_{R_{r}} \mathbf{F}_{p}$ are surjective.

One of the miraculous properties of the cohomology sequence (1.2.1) is that it is compatible with base change, in the sense that $H\left(X_{r} / R_{r}\right) \otimes_{R_{r}} \mathbf{F}_{p}$ may be functorially identified with the hypercohomology $H^{\bullet}\left(\bar{X}_{r} / \mathbf{F}_{p}\right)$ of the two term complex $d: \mathscr{O}_{\bar{X}_{r}} \rightarrow \omega_{\bar{X}_{r} / \mathbf{F}_{p}}$ on the special fiber $\bar{X}_{r}:=X_{r} \times_{R_{r}} \mathbf{F}_{p}$, where

\footnotetext{
${ }^{2}$ That is, $\psi(\sigma \tau)=\psi(\sigma) \cdot \sigma \psi(\tau)$ for all $\sigma, \tau \in \Gamma$,
} 
$\omega_{\bar{x}_{r} / \mathbf{F}_{p}}$ is the relative dualizing sheaf; see Lemma A.14. Thus, we obtain a functorial identification of $e_{r}^{*} H\left(\mathcal{X}_{r} / R_{r}\right) \otimes_{R_{r}} \mathbf{F}_{p}$ with the short exact sequence

$$
0 \longrightarrow e_{r}^{*} H^{0}\left(\bar{X}_{r}, \omega_{\bar{x}_{r} / \mathbf{F}_{p}}\right) \longrightarrow e_{r}^{*} H^{1}\left(\bar{X}_{r} / \mathbf{F}_{p}\right) \longrightarrow e_{r}^{*} H^{1}\left(\bar{X}_{r}, \mathscr{O}_{\bar{x}_{r}}\right) \longrightarrow 0
$$

arising from the degeneration of the "Hodge to de Rham" spectral sequence that computes the hypercohomology $H^{1}\left(\bar{X}_{r} / \mathbf{F}_{p}\right)$. Thus, in order to prove (1) and (2) above, we are reduced to a problem about the cohomology (1.3.1) of the characteristc $p$ schemes $\bar{X}_{r}$.

Now the fact that $\bar{X}_{r}$ is reduced plays a critical role, as it allows us to use Rosenlicht's explicit description of the relative dualizing sheaf for a reduced curve to determine the structure of the exact sequence (1.3.1). Rosenlicht's theory allows us to identify $H^{0}\left(\bar{X}_{r}, \omega_{\bar{x}_{r} / \mathbf{F}_{p}}\right)$ with a certain subspace of meromorphic differentials on the normalization $\bar{x}_{r}^{\mathrm{n}}$ of $\bar{X}_{r}$. This normalization is a disjoint union of smooth and proper "Igusa" curves, and has two "privileged" irreducible components: namely the component $I_{r}^{\infty}$ which meets the $\infty$-cuspidal section of $\mathcal{X}_{r}$, and $I_{r}^{0}$, which meets the 0 -cuspidal section. Using the description of the correspondences $U_{p}$ and $U_{p}^{*}$ on $\bar{X}_{r}^{\mathrm{n}}$ due to Ulmer [Ulmer], and recorded in Proposition B.25, together with the fact that pullback by Frobenius kills differential forms in characteristic $p$, we calculate that these correspondences "contract" the space of meromorphic differentials on $\bar{X}_{r}^{\mathrm{n}}$ onto the components $I_{r}^{\star}$. More precisely, we prove in $\S 2.3$ that pullback of meromorphic differentials along the canonical closed immersions $I_{r}^{\star} \hookrightarrow \bar{X}_{r}^{\mathrm{n}}$ induce natural isomorphisms

$$
e_{r}^{*} H^{0}\left(\bar{X}_{r}, \omega_{\bar{x}_{r} / \mathbf{F}_{p}}\right) \simeq H^{0}\left(I_{r}^{\infty}, \Omega_{I_{r}^{\infty}}^{1}(\underline{\mathrm{ss}})\right)^{V_{\text {ord }}} \quad \text { and } \quad e_{r} H^{0}\left(\bar{X}_{r}, \omega_{\bar{X}_{r} / \mathbf{F}_{p}}\right) \simeq H^{0}\left(I_{r}^{0}, \Omega_{I_{r}^{0}}^{1}(\underline{\mathrm{ss}})\right)^{V_{\text {ord }}},
$$

where the superscript of " $V_{\text {ord" }}$ means the $V$-ordinary part of cohomology, by definition the maximal subspace on which the Cartier operator $V$ acts invertibly. Applying Grothendieck duality - which swaps the idempotent $e_{r}$ with $e_{r}^{*}$ and the Cartier operator with pullback by absolute Frobenius - to the second of these isomorphisms yields functorial identifications

$$
e_{r}^{*} H^{1}\left(\bar{X}_{r}, \mathscr{O}_{\bar{X}_{r}}\right) \simeq e_{r} H^{0}\left(\bar{X}_{r}, \omega_{\bar{X}_{r} / \mathbf{F}_{p}}\right)^{\vee} \simeq\left(H^{0}\left(I_{r}^{0}, \Omega_{I_{r}^{0}}^{1}(\underline{\mathrm{ss}})\right)^{V_{\text {ord }}}\right)^{\vee} \simeq H^{1}\left(I_{r}^{0}, \mathscr{O}_{I_{r}^{0}}(-\underline{\mathrm{ss}})\right)^{F_{\text {ord }}}
$$

where the superscript of " $F_{\text {ord }}$ " denotes the maximal subspace on which pullback by absolute Frobenius is invertible. From the functoriality of (1.3.3), we deduce that pullback by absolute Frobenius is an isomorphism on $e_{r}^{*} H^{1}\left(\bar{X}_{r}, \mathscr{O}_{\bar{x}_{r}}\right)$, and it follows (recalling again that pullback by Frobenius kills differentials) that (1.3.1) is functorially split by Frobenius. In this way, and via the isomorphisms (1.3.2)-(1.3.3), the structure of the cohomology exact sequence (1.3.1) is entirely captured by the space of $V$-ordinary meromorphic differential forms on the Igusa towers $\left\{I_{r}^{\star}\right\}_{r}$ for $\star=0, \infty$.

Nakajima's beautiful equivariant Deuring-Shafarevich formula (Proposition 2.1.7 below), applied to the $\Delta_{1} / \Delta_{r}$-cover $I_{r}^{\star} \rightarrow I_{1}^{\star}$, allows us to conclude that the right side of each isomorphism in (1.3.2) is free of rank $d$ as a module over the group $\operatorname{ring} \mathbf{F}_{p}\left[\Delta_{1} / \Delta_{r}\right]$. We recall the context and statement of this key result in $\S 2.1$, and apply it to the cohomology of the Igusa tower $I_{r}^{\star}$ in $\S 2.2$. It then follows from the identifications (1.3.2)-(1.3.3) that the flanking terms of the exact sequence (1.3.1) are likewise free of rank $d$ over $\mathbf{F}_{p}\left[\Delta_{1} / \Delta_{r}\right]$, from which we deduce (using, for example, the functorial Frobenius splitting of (1.3.1) noted above) that the middle term $e_{r}^{*} H^{1}\left(\bar{X}_{r} / \mathbf{F}_{p}\right)$ of this sequence is then free of rank $2 d$ over $\mathbf{F}_{p}\left[\Delta_{1} / \Delta_{r}\right]$, which establishes the key claim (1) above.

To prove (2), and thus complete the proof of Theorem 1.2.1, we use the established Frobenius splitting of (1.3.1) and the identification of its flanking terms with the cohomology of the Igusa tower provided by (1.3.2)-(1.3.3), together with Grothendieck duality, to reduce the asserted surjectivity of 
$\rho_{*} \otimes 1$ to the following claim: the canonical pullback maps

$$
\begin{gathered}
\rho^{*}: H^{0}\left(I_{r+1}^{0}, \Omega_{I_{r+1}^{0}}^{1}(\underline{\mathrm{ss}})\right) \longrightarrow H^{0}\left(I_{r}^{0}, \Omega_{I_{r}^{0}}^{1}(\underline{\mathrm{s}})\right) \\
\rho^{*}: H^{1}\left(I_{r+1}^{\infty}, \mathscr{O}_{I_{r+1}^{\infty}}(-\underline{\mathrm{ss}})\right) \longrightarrow H^{1}\left(I_{r}^{\infty}, \mathscr{O}_{I_{r}^{\infty}}(-\underline{\mathrm{ss}})\right)
\end{gathered}
$$

attached to the degeneracy mapping $\rho: I_{r+1}^{\star} \rightarrow I_{r}^{\star}$ are both injective. The injectivity of (1.3.4a) is clear, as $\rho$ is generically étale, while we prove that (1.3.4b) is injective in Proposition 2.2.1 and Lemma 2.2.2 by interpreting classes in $H^{1}$ as line bundles and using the fact that the degeneracy mapping $\rho$ totally ramifies over every supersingular point.

With Theorem 1.2.1 established, we show as part of our commutative algebra formalism in Lemma 3.1.4 that the proof of Theorem 1.2.2 may then be reduced to the existence of certain autoduality pairings on $e_{r}^{*} H^{1}\left(\mathcal{X}_{r} / R_{r}^{\prime}\right)$ that are compatible in a precise sense with change in $r$ (see (3.1.3) for the exact condition). Using the fact that the canonical $R_{r}^{\prime}$-lattice $H^{1}\left(X_{r} / R_{r}^{\prime}\right)$ in the de Rham cohomology of the generic fiber $X_{r}$ over $K_{r}^{\prime}$ is preserved by the standard cup-product autoduality, we are able to "twist" the restriction of this pairing to $e_{r}^{*} H^{1}\left(X_{r} / R_{r}^{\prime}\right)$ by an appropriate power of $U_{p}^{*}$ and the Atkin-Lehner involution (which explains why we must work over $R_{r}^{\prime}=R_{r}\left[\mu_{N}\right]$ rather than $R_{r}$ itself) to obtain a perfect self-pairing that satisfies the required compatibility condition.

1.4. Notation. If $\varphi: A \rightarrow B$ is any map of rings, we will often write $M_{B}:=M \otimes_{A} B$ for the $B$-module induced from an $A$-module $M$ by extension of scalars. When we wish to specify $\varphi$, we will write $M \otimes_{A, \varphi} B$. Likewise, if $\varphi: T^{\prime} \rightarrow T$ is any morphism of schemes, for any $T$-scheme $X$ we denote by $X_{T^{\prime}}$ the base change of $X$ along $\varphi$. If $f: X \rightarrow Y$ is any morphism of $T$-schemes, we will write $f_{T^{\prime}}: X_{T^{\prime}} \rightarrow Y_{T^{\prime}}$ for the morphism of $T^{\prime}$-schemes obtained from $f$ by base change along $\varphi$. When $T=\operatorname{Spec}(R)$ and $T^{\prime}=\operatorname{Spec}\left(R^{\prime}\right)$ are affine, we abuse notation and write $X_{R^{\prime}}$ or $X \times_{R} R^{\prime}$ for $X_{T^{\prime}}$. We will frequently work with schemes over a discrete valuation ring $R$. We will often write $x, y, \ldots$ for schemes over $\operatorname{Spec}(R)$, and will generally use $X, Y, \ldots$ (respectively $\bar{x}, \bar{y}, \ldots$ ) for their generic (respectively special) fibers.

The following notation will be in effect throughout this article and its sequel [Cais3]. We always assume that $p>2$ is a fixed prime and $N$ is a fixed positive integer with $p \nmid N$ and $N p>4$. We set $K_{r}:=\mathbf{Q}_{p}\left(\mu_{p^{r}}\right)$ and $R_{r}:=\mathbf{Z}_{p}\left[\mu_{p^{r}}\right]$, and put $K_{r}^{\prime}:=K_{r}\left(\mu_{N}\right)$ and $R_{r}^{\prime}:=R_{r}\left[\mu_{N}\right]$. We choose, once and for all, a compatible sequence $\left\{\varepsilon^{(r)}\right\}_{r \geq 0}$ of primitive $p^{r}$-th roots of unity. As in $\S 1.2$, for $r \geq 1$, we denote by $X_{r}:=X_{1}\left(N p^{r}\right)$ the canonical model over $\mathbf{Q}$ with rational cusp at $i \infty$ of the modular curve arising as the quotient of the extended upper half-plane by the congruence subgroup $\Gamma_{1}\left(N p^{r}\right)$. There are two natural degeneracy mappings $\rho, \sigma: X_{r+1} \rightrightarrows X_{r}$ of curves over $\mathbf{Q}$ induced by the self-maps of the upper half-plane $\rho: \tau \mapsto \tau$ and $\sigma: \tau \mapsto p \tau$. We write $J_{r}:=\operatorname{Pic}_{X_{r} / \mathbf{Q}}^{0}$ for the Jacobian of $X_{r}$ over $\mathbf{Q}$ and $\mathfrak{H}_{r}(\mathbf{Z})$ for the $\mathbf{Z}$-subalgebra of $\operatorname{End}_{\mathbf{Q}}\left(J_{r}\right)$ generated by the Hecke operators $\left\{T_{\ell}\right\}_{\ell \nmid N p},\left\{U_{\ell}\right\}_{\ell \mid N p}$ and the

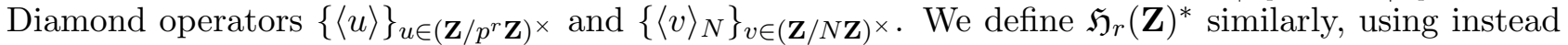
the "transpose" Hecke and diamond operators, and set $\mathfrak{H}_{r}:=\mathfrak{H}_{r}(\mathbf{Z}) \otimes_{\mathbf{Z}} \mathbf{Z}_{p}$ and $\mathfrak{H}_{r}^{*}:=\mathfrak{H}_{r}(\mathbf{Z})^{*} \otimes_{\mathbf{Z}} \mathbf{Z}_{p}$; see Definitions A.15 and B.26, and the surrounding discussion. As usual, $e_{r} \in \mathfrak{H}_{r}$ and $e_{r}^{*} \in \mathfrak{H}_{r}^{*}$ are the idempotents of these semi-local $\mathbf{Z}_{p}$-algebras corresponding to the Atkin operators $U_{p}$ and $U_{p}^{*}$, respectively. We put $e:=\left(e_{r}\right)_{r}$ and $e^{*}:=\left(e_{r}^{*}\right)_{r}$ for the induced idempotents of the "big" $p$-adic Hecke algebras $\mathfrak{H}:=\lim _{r} \mathfrak{H}_{r}$ and $\mathfrak{H}^{*}:=\lim _{r} \mathfrak{H}_{r}^{*}$, with the projective limits formed using the transition mappings induced by the maps on Jacobians $J_{r} \rightrightarrows J_{r^{\prime}}$ for $r^{\prime} \geq r$ arising (via Picard functoriality) from $\sigma$ and $\rho$, respectively. We will consistently view $\mathfrak{H}^{*}$ (respectively $\mathfrak{H}$ ) as a $\Lambda$-module via the $\mathbf{Z}_{p^{-}}$linear map $\Lambda \hookrightarrow \mathfrak{H}^{*}$ (respectively $\Lambda \hookrightarrow \mathfrak{H}$ ) sending $u \in \Delta_{1}$ to $\langle u\rangle^{*}$ (respectively $\langle u\rangle$ ). Let $w_{r}$ be 
the Atkin-Lehner "involution" of $X_{r}$ over $\mathbf{Q}\left(\mu_{N p^{r}}\right)$ corresponding to a choice of primitive $N p^{r}$-th root of unity as in Appendix B and simply write $w_{r}$ for the automorphism $\operatorname{Alb}\left(w_{r}\right)$ of $J_{r}$ over $\mathbf{Q}\left(\mu_{N p^{r}}\right)$ induced by Albanese functoriality. We note that for any Hecke operator $T \in \mathfrak{H}_{r}(\mathbf{Z})$ one has the relation $w_{r} T=T^{*} w_{r}$ as endomorphisms of $J_{r}$ over $\mathbf{Q}\left(\mu_{N p^{r}}\right)$; see [Tilouine, pg. 336], [Ohta1, 2.1.8], or [MW2, Chapter 2, $\S 5.6(\mathrm{c})]$.

1.5. Acknowledgements. It is a pleasure to thank Brian Conrad, Adrian Iovita, Joseph Lipman, and Romyar Sharifi for enlightening conversations and correspondence, and Doug Ulmer for supplying the proof of Lemma B.4. This paper owes a great deal to the work of Masami Ohta, to whom this paper is dedicated, and I heartily thank him for graciously hosting me during a visit to Tokai University in August 2009.

\section{Contents}

1. Introduction 1

1.1. Motivation 1

1.2. Results 2

1.3. Overview of the article 4

1.4. Notation 6

1.5. Acknowledgements

2. $\quad$ de Rham Cohomology of Modular Curves in characteristic $p \quad 7$

2.1. The Cartier operator $\quad 8$

2.2. The Igusa tower 11

2.3. Structure of the ordinary part of $H^{0}\left(\bar{X}_{r}, \omega_{\bar{x}_{r} / \mathbf{F}_{p}}\right) \quad 14$

3. Ordinary $\Lambda$-adic de Rham cohomology 23

3.1. The formalism of towers 23

3.2. Ordinary families of de Rham cohomology 27

3.3. Relation to ordinary $\Lambda$-adic modular forms 30

Appendices 33

A. Dualizing sheaves and cohomology 33

B. Integral models of modular curves $\quad 40$

References

\section{De Rham Cohomology of Modular Curves in characteristic $p$}

We keep the notation of $\S 1.4$, and refer to appendices $\mathrm{A}$ and $\mathrm{B}$ for the background material on integral models of modular curves and "de Rham" cohomology for non-smooth curves that will be used throughout this section. Let $X_{r}$ be the Katz-Mazur model of $X_{1}\left(N p^{r}\right)$ over $R_{r}:=\mathbf{Z}_{p}\left[\mu_{p^{r}}\right]$ provided by Definition B.6. Due to Proposition B.3, the scheme $X_{r}$ is regular and proper flat of pure relative dimension one over $R_{r}$ with fibers that are geometrically connected and reduced thanks to Proposition B.14; it is therefore a curve in the sense of Definition A.1 due to Corollary A.3.

Denote by $\bar{X}_{r}:=X_{r} \times_{R_{r}} \mathbf{F}_{p}$ the special fiber of $X_{r}$. As $\bar{X}_{r}$ is again a curve in the sense of Definition A.1, the relative dualizing sheaf $\omega_{\bar{x}_{r} / \mathbf{F}_{p}}$ exists and is a line bundle on $\bar{X}_{r}$, and there is a canonical twoterm complex d : $\mathscr{O}_{\bar{x}_{r}} \rightarrow \omega_{\bar{x}_{r} / \mathbf{F}_{p}}$ whose hypercohomology provides a well-behaved version of de Rham cohomology for non-smooth curves such as $\bar{X}_{r}$; see Appendix A. In particular, thanks to Proposition 
A.11, the first hypercohomology of this complex sits in a functorial short-exact sequence of $\mathbf{F}_{p}$-vector spaces

$$
0 \longrightarrow H^{0}\left(\bar{X}_{r}, \omega_{\bar{x}_{r} / \mathbf{F}_{p}}\right) \longrightarrow H^{1}\left(\bar{X}_{r} / \mathbf{F}_{p}\right) \longrightarrow H^{1}\left(\bar{X}_{r}, \mathscr{O}_{\bar{x}_{r}}\right) \longrightarrow 0
$$

which we denote by $H\left(\bar{X}_{r} / \mathbf{F}_{p}\right)$.

In this section, we will use Rosenlicht's explicit description of the dualizing sheaf to compute the ordinary part of the cohomology $H\left(\bar{X}_{r} / \mathbf{F}_{p}\right)$ in terms of the de Rham cohomology of the Igusa tower, which we analyze in $\S 2.2$. The crucial ingredient in our analysis is Nakajima's beautiful equivariant Deuring-Shafarevich formula, which we recall first.

2.1. The Cartier operator. Fix a perfect field $k$ of characteristic $p>0$ and write $\varphi: k \rightarrow k$ for the $p$-power Frobenius map. In this section, we recall the basic theory of the Cartier operator for a smooth and proper curve over $k$. As we will only need the theory in this limited setting, we will content ourselves with a somewhat ad hoc formulation of it. Our exposition follows [Serre, §10], but the reader may consult [Oda, §5.5] or [Cartier] for a more general treatment.

Let $X$ be a smooth and proper curve over $k$ and write $F: X \rightarrow X$ for the absolute Frobenius map; it is finite and flat and is a morphism over the endomorphism of $\operatorname{Spec}(k)$ induced by $\varphi$. Moreover, if $\mathscr{L}$ is a line bundle on $X$, then one has a canonical isomorphism $F^{*} \mathscr{L} \simeq \mathscr{L}^{\otimes p}$ of line bundles. ${ }^{3}$ Let $D$ be an effective Cartier $\left(=\right.$ Weil) divisor on $X$ over $k$, and write $\mathscr{O}_{X}(-D)$ for the coherent (invertible) ideal sheaf determined by $D$. The pullback map $F^{*}: \mathscr{O}_{X} \rightarrow F_{*} \mathscr{O}_{X}$ carries the ideal sheaf $\mathscr{O}_{X}(-n D) \subseteq \mathscr{O}_{X}$ into $F_{*} \mathscr{O}_{X}(-n p D)$, so we obtain a canonical $\varphi$-semilinear pullback map on cohomology

$$
F^{*}: H^{1}\left(X, \mathscr{O}_{X}(-n D)\right) \longrightarrow H^{1}\left(X, \mathscr{O}_{X}(-n p D)\right) \text {. }
$$

By Grothendieck-Serre duality, (2.1.1) gives a $\varphi^{-1}$-semilinear "trace" map of $k$-vector spaces

$$
V:=F_{*}: H^{0}\left(X, \Omega_{X / k}^{1}(n p D)\right) \longrightarrow H^{0}\left(X, \Omega_{X / k}^{1}(n D)\right) .
$$

which, thanks to the very construction of the duality isomorphism [Conrad, 3.4.10], coincides with the map induced by Grothendieck's trace morphism on dualizing sheaves attached to the finite map $F$ [Conrad, 2.7.36].

Proposition 2.1.1. Let $X / k$ be a smooth and proper curve, $D$ an effective Cartier divisor on $X$, and $n$ a nonnegative integer; for $x \in \mathbf{R}$ we write $\lceil x\rceil$ for the least integer $m$ satisfying $m \geq x$.

(1) There is a unique $\varphi^{-1}$-linear endomorphism $V:=F_{*}$ of $H^{0}\left(X, \Omega_{X / k}^{1}(n D)\right)$ which is dual, via Grothendieck-Serre duality, to pullback by absolute Frobenius on $H^{1}\left(X, \mathscr{O}_{X}(-n D)\right)$.

(2) The map $V$ "improves poles" in the sense that it factors through the canonical inclusion

$$
H^{0}\left(X, \Omega_{X / k}^{1}\left(\left\lceil\frac{n}{p}\right\rceil D\right)\right) \longleftrightarrow H^{0}\left(X, \Omega_{X / k}^{1}(n D)\right) .
$$

(3) If $\rho: Y \rightarrow X$ is any finite morphism of smooth proper curves over $k$, and $\rho^{*} D$ is the pullback of $D$ to $Y$, then the induced pullback and trace maps

$$
H^{0}\left(Y, \Omega_{Y / k}^{1}\left(n \rho^{*} D\right)\right) \underset{\rho^{*}}{\stackrel{\rho_{*}}{\rightleftarrows}} H^{0}\left(X, \Omega_{X / k}^{1}(n D)\right)
$$

\footnotetext{
${ }^{3}$ This useful description of $F^{*} \mathscr{L}$ justifies our choice to use the absolute Frobenius map rather than the relative Frobenius map $F_{X / k}: X \rightarrow X^{(p)}$, which does not enjoy any analogous property.
} 
commute with $V$.

(4) Let $x$ be any $k$-point point of $X$, choose a uniformizer $t \in \mathscr{O}_{X, x}$, and for any meromorphic differential $\eta$ on $X$, denote by $\eta_{x}$ the image of $\eta$ under $\Omega_{k(X) / k}^{1} \hookrightarrow \Omega_{k(X) / k}^{1} \otimes_{\mathscr{O}_{X, x}} \widehat{\mathscr{O}}_{X, x}=k((t)) \mathrm{d} t$.

$$
\text { If } \eta_{x}=\sum_{n} a_{n} t^{n} \mathrm{~d} t \quad \text { then } \quad V(\eta)_{x}=\sum_{n \equiv-1 \bmod p} \varphi^{-1}\left(a_{n}\right) t^{(n+1) / p-1} \mathrm{~d} t .
$$

(5) With hypotheses and notation as in (4), we have $\operatorname{res}_{x}(V \eta)^{p}=\operatorname{res}_{x}(\eta)$ where $\operatorname{res}_{x}$ is the canonical "residue at $x$ map" on meromorphic differentials, determined by the condition $\operatorname{res}_{x}(\eta):=a_{-1}$.

Proof. Both (1) and (2) follow from our discussion, while (3) follows (via duality) from the fact that the $p$-power map commutes with any ring homomorphism in characteristic $p$.

To prove (4), we work locally at $x$ and use the fact noted above that $V$ is induced by Grothendieck's trace map $\operatorname{Tr}_{F}: F_{*} \Omega_{X / k}^{1} \rightarrow \Omega_{X / k}^{1}$ attached to $F$. We may find a Zariski open neighborhood $U$ of $x$ in $X$ that admits a finite étale map $U \rightarrow \mathbf{A}_{k}^{1}$ carrying $x$ to the origin, and since the formation of $\operatorname{Tr}_{F}$ is compatible with étale localization, we may reduce to checking the proposed formula for $X=\mathbf{A}_{k}^{1}$ at the origin. As $\operatorname{Tr}_{F}$ is compatible with base change (see [Conrad, pgs. 97-99]), we may further reduce to the case $k=\mathbf{F}_{p}$. Again invoking compatibility with base change, this map is the reduction modulo $p$ of the Grothendieck trace mapping attached to the standard lift of Frobenius on $\mathbf{A}_{\mathbf{Z}_{p}}^{1}$ given at the level of coordinate rings by the $\mathbf{Z}_{p}$-algebra map $\Phi: \mathbf{Z}_{p}[T] \rightarrow \mathbf{Z}_{p}[T]$ sending $T$ to $T^{p}$. Appealing now to the explicit description of Grothendieck's trace mapping afforded by [Conrad, 2.7.41], we find that for any $b \in \mathbf{Z}_{p}((T)):=\mathbf{Z}_{p} \llbracket T \rrbracket[1 / T]$, one has

$$
\operatorname{Tr}_{F}(b \mathrm{~d} \Phi(T))=\operatorname{Tr}_{\Phi}(b) \mathrm{d} T,
$$

where $\operatorname{Tr}_{\Phi}: \mathbf{Z}_{p}((T)) \rightarrow \mathbf{Z}_{p}((T))$ is the standard ring-theoretic trace mapping attached to $\Phi$ (which realizes $\mathbf{Z}_{p}((T))$ as a free module of rank $p$ over itself $)$. The computation

$$
\operatorname{Tr}_{\Phi}\left(T^{j}\right)= \begin{cases}0 & \text { if } j \not \equiv 0 \bmod p \\ p T^{j / p} & \text { otherwise }\end{cases}
$$

is a standard exercise in linear algebra, whence we obtain from (2.1.3) the identity

$$
\operatorname{Tr}_{F}\left(\sum_{j} a_{j} T^{j} p T^{p-1} \mathrm{~d} T\right)=\sum_{j \equiv 0 \bmod p} p a_{j} T^{j / p} \mathrm{~d} T
$$

which, after canceling the factor of $p$ on either side (remember that we are now working in a rank-1 free $\mathbf{Z}_{p}((T))$-module!), is equivalent to the desired formula.

Finally, (5) is an immediate consequence of (4) and the given explicit description of res . $_{x}$.

Remark 2.1.2. As a sort of complement to Proposition 2.1.1 (5), we have the following: assume that $\rho: Y \rightarrow X$ is a finite and generically étale morphism of smooth curves over an algebraically closed field $k$. Then

$$
\sum_{y \in \rho^{-1}(x)} \operatorname{res}_{y}(\eta)=\operatorname{res}_{x}\left(\rho_{*} \eta\right)
$$

Indeed, as $\rho$ is generically étale, we have $\Omega_{k(Y) / k}^{1}=k(Y) \otimes_{k(X)} \Omega_{k(X) / k}^{1}$, so the claimed formula follows from [Tate2, Theorem 4] and the explicit description of Grothendieck's trace morphism provided by [Conrad, 2.7.41], together with the facts that the isomorphism $H^{1}\left(X, \Omega_{X / k}^{1}\right) \rightarrow k$ induced by the residue 
map coincides with the negative of Grothendieck's trace isomorphism, and the latter is compatible with compositions; see Appendix B and Corollary 3.6.6 of [Conrad].

We recall the following (generalization of a) well-known lemma of Fitting:

Lemma 2.1.3. Let $A$ be a commutative ring, $\varphi$ an automorphism of $A$, and $M$ an $A$-module equipped with a $\varphi$-semilinear endomorphism $F: M \rightarrow M$. Assume that one of the following holds:

(1) $M$ is a finite length A-module.

(2) $A$ is a complete noetherian adic ring, with ideal of definition $I \subseteq A$, and $M$ is a finitely generated A-module.

Then there is a unique direct sum decomposition

$$
M=M^{F_{\text {ord }}} \oplus M^{F_{\text {nil }},}
$$

where $M^{F_{\text {ord }}}$ is the maximal $F$-stable submodule of $M$ on which $F$ is bijective, and $M^{F_{\text {nil }}}$ is the maximal $F$-stable submodule of $M$ on which $F$ is (topologically) nilpotent. The assignment $M \rightsquigarrow M^{F_{\star}}$ for $\star=$ ord, nil is an exact functor on the category of (left) $A[F]$-modules verifying (1) or (2).

Proof. For the proof in case (1), we refer to [Lazard, VI, 5.7], and just note that one has:

$$
M^{F_{\text {ord }}}:=\bigcap_{n \geq 0} \operatorname{im}\left(F^{n}\right) \text { and } \quad M^{F_{\text {nil }}}:=\bigcup_{n \geq 0} \operatorname{ker}\left(F^{n}\right),
$$

where one uses that $\varphi$ is an automorphism to know that the image and kernel of $F^{n}$ are $A$-submodules of $M$. It follows immediately from this that the association $M \rightsquigarrow M^{F_{\star}}$ is a functor from the category of left $A[F]$-modules of finite $A$-length to itself. It is an exact functor because the canonical inclusion $M^{F_{\star}} \rightarrow M$ is an $A[F]$-direct summand. In case (2), our hypotheses ensure that $M / I^{n} M$ is a noetherian and Artinian $A$-module, and hence of finite length, for all $n$. Our assertions in this situation then follow immediately from (1), via the uniqueness of (2.1.5), together with fact that $M$ is finite as an $A$-module, and hence $I$-adically complete (as $A$ is).

We apply 2.1.3 to the $k$-vector space $M:=H^{0}\left(X, \Omega_{X / k}^{1}\right)$ equipped with the $\varphi^{-1}$-semilinear map $V$ :

Definition 2.1.4. The $k[V]$-module $H^{0}\left(X, \Omega_{X / k}^{1}\right)^{V_{\text {ord }}}$ is called the $V$-ordinary subspace of holomorphic differentials on $X$. The integer $\gamma_{X}:=\operatorname{dim}_{k} H^{0}\left(X, \Omega_{X / k}^{1}\right)^{V_{\text {ord }}}$ is called the Hasse-Witt invariant of $X$.

Remark 2.1.5. Let $D$ be any effective Cartier divisor. Since $V:=F_{*}$ and $F:=F^{*}$ are adjoint under the canonical perfect $k$-pairing between $H^{0}\left(X, \Omega_{X / k}^{1}(D)\right)$ and $H^{1}\left(X, \mathscr{O}_{X}(-D)\right)$, this pairing restricts to a perfect duality pairing

$$
H^{0}\left(X, \Omega_{X / k}^{1}(D)\right)^{V_{\text {ord }}} \times H^{1}\left(X, \mathscr{O}_{X}(-D)\right)^{F_{\text {ord }}} \longrightarrow k .
$$

In particular (taking $D=0$ ) we also have $\gamma_{X}=\operatorname{dim}_{k} H^{1}\left(X, \mathscr{O}_{X}\right)^{F_{\text {ord }}}$.

The following "control lemma" is a manifestation of the fact that the Cartier operator improves poles (Proposition 2.1.1, (2)):

Lemma 2.1.6. Let $X$ be a smooth and proper curve over $k$ and $D$ an effective Cartier divisor on $X$. Considering $D$ as a closed subscheme of $X$, we write $D_{\text {red }}$ for the associated reduced closed subscheme. 
(1) For all positive integers $n$, the canonical morphism

$$
H^{0}\left(X, \Omega_{X / k}^{1}\left(D_{\text {red }}\right)\right) \rightarrow H^{0}\left(X, \Omega_{X / k}^{1}(n D)\right)
$$

induces a natural isomorphism on $V$-ordinary subspaces.

(2) For each positive integer $n$, the canonical map

$$
H^{1}\left(X, \mathscr{O}_{X}(-n D)\right) \rightarrow H^{1}\left(X, \mathscr{O}_{X}\left(-D_{\text {red }}\right)\right)
$$

induces a natural isomorphism on F-ordinary subspaces.

(3) The identifications in (1) and (2) are canonically k-linearly dual, via Remark 2.1.5.

Proof. This follows immediately from Proposition 2.1.1 (2) and Remark 2.1.5.

Now let $\pi: Y \rightarrow X$ be a finite map of smooth, proper and geometrically connected curves over $k$ that is generically étale and Galois with group $G$ that is a $p$-group. Let $D_{X}$ be any effective Cartier divisor on $X$ over $k$ with support containing the ramification locus of $\pi$, and put $D_{Y}=\pi^{*} D_{X}$. As in Lemma 2.1.6, denote by $D_{X \text {,red }}$ and $D_{Y \text {,red }}$ the underlying reduced closed subschemes; as $D_{Y \text {,red }}$ is $G$-stable, the $k$-vector spaces $H^{0}\left(Y, \Omega_{Y / k}^{1}\left(n D_{Y \text {,red }}\right)\right)$ and $H^{1}\left(Y, \mathscr{O}_{Y}\left(-n D_{Y \text {,red }}\right)\right)$ are canonically $k[G]$ modules for any $n \geq 1$. The following equivariant Deuring-Shafarevich formula of Nakajima is the key to the proofs of our structure theorems for $\Lambda$-modules:

Proposition 2.1.7 (Nakajima). Assume that $\pi$ is ramified, let $\gamma_{X}$ be the Hasse-Witt invariant of $X$ and set $d:=\gamma_{X}-1+\operatorname{deg}\left(D_{X, \text { red }}\right)$. Then for each positive integer $n$ :

(1) The $k[G]$-module $H^{0}\left(Y, \Omega_{Y / k}^{1}\left(n D_{Y, \text { red }}\right)\right)^{V_{\text {ord }}}$ is free of rank $d$ and independent of $n$.

(2) The $k[G]$-module $H^{1}\left(Y, \mathscr{O}_{Y}\left(-n D_{Y \text {,red }}\right)\right)^{F_{\text {ord }}}$ is naturally isomorphic to the contragredient of $H^{0}\left(Y, \Omega_{Y / k}^{1}\left(n D_{Y, \text { red }}\right)\right)^{V_{\text {ord }}}$; as such, it is $k[G]$-free of rank $d$ and independent of $n$.

Proof. The independence of $n$ is simply Lemma 2.1.6; using this, the first assertion is then equivalent to Theorem 1 of [Nakajima]. The second assertion is immediate from Remark 2.1.5, once one notes that for $g \in G$ one has the identity $g_{*}=\left(g^{-1}\right)^{*}$ on cohomology (since $g_{*} g^{*}=\operatorname{deg} g=\mathrm{id}$ ), so $g^{*}$ and $\left(g^{-1}\right)^{*}$ are adjoint under the duality pairing (2.1.6).

2.2. The Igusa tower. We apply Proposition 2.1.7 to the Igusa tower. Recall (Definition B.12) that for $r \geq 0$ we write $\operatorname{Ig}_{r}$ for the compactified moduli scheme classifying elliptic curves over $\mathbf{F}_{p^{-}}$ schemes equipped with a level- $p^{r}$ Igusa structure and a $\mu_{N}$-structure as in Definition B.10. Then $\operatorname{Ig}_{r}$ is a smooth, projective and geometrically irreducible curve over $\mathbf{F}_{p}$ by Proposition B.11, and comes equipped with a canonical degeneracy map $\rho: \operatorname{Ig}_{r} \rightarrow \operatorname{Ig}_{1}$ defined moduli-theoretically by (B.7) that is finite étale outside $\underline{\mathrm{ss}}^{4}=\underline{\mathrm{ss}}_{r}$ and totally (wildly) ramified over $\underline{\mathrm{ss}}_{1}$. In this way, $\mathrm{Ig}_{r}$ is a branched cover of $\operatorname{Ig}_{1}$ with group $\Delta_{1} / \Delta_{r}$, so that the cohomology groups $H^{0}\left(\operatorname{Ig}_{r}, \Omega_{\operatorname{Ig}_{r} / \mathbf{F}_{p}}^{1}(\underline{\mathrm{ss}})\right)$ and $H^{1}\left(\operatorname{Ig}_{r}, \mathscr{O}_{\operatorname{Ig}_{r}}(-\underline{\mathrm{ss}})\right)$ are naturally $\mathbf{F}_{p}\left[\Delta_{1} / \Delta_{r}\right]$-modules. Note that we have $\operatorname{Ig}_{0}=X_{1}(N)_{\mathbf{F}_{p}}$, the compactified modular curve over $\mathbf{F}_{p}$ classifying elliptic curves with a $\mu_{N}$-structure.

Proposition 2.2.1. Let $r \geq 1$ be an integer, write $\gamma$ for the $p$-rank of $\operatorname{Pic}_{X_{1}(N) / \mathbf{F}_{p}}^{0}$, and set $\delta:=\operatorname{deg} \underline{\operatorname{ss}}$.

(1) The $\mathbf{F}_{p}\left[\Delta_{1} / \Delta_{r}\right]$-modules $H^{0}\left(\operatorname{Ig}_{r}, \Omega_{\operatorname{Ig}_{r} / \mathbf{F}_{p}}^{1}(\underline{\mathrm{ss}})\right)^{V_{\text {ord }}}$ and $H^{1}\left(\operatorname{Ig}_{r}, \mathscr{O}_{\operatorname{Ig}_{r}}(-\underline{\mathrm{ss}})\right)^{F_{\text {ord }}}$ are both free of rank $d:=\gamma+\delta-1$. Each is canonically isomorphic to the contragredient of the other.

\footnotetext{
${ }^{4}$ We will frequently write simply $\underline{\mathrm{ss}}$ for the divisor $\underline{\mathrm{sS}}_{r}$ on $\mathrm{Ig}_{r}$ when $r$ is clear from context.
} 
(2) For any positive integer $s \leq r$, the canonical trace mapping associated to $\rho: \operatorname{Ig}_{r} \rightarrow \operatorname{Ig}_{s}$ induces natural isomorphisms of $\mathbf{F}_{p}\left[\Delta_{1} / \Delta_{s}\right]$-modules

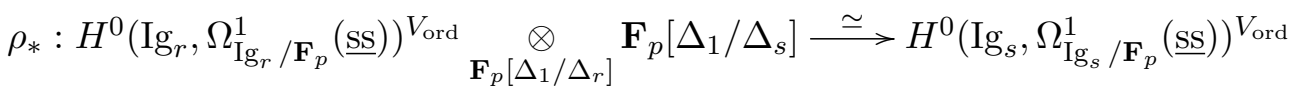

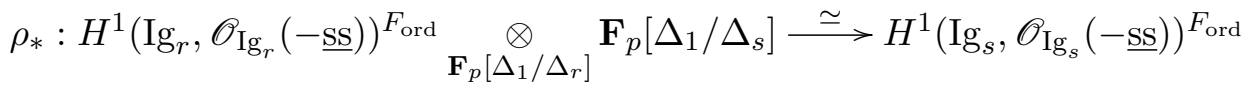

In order to prove Proposition 2.2.1, we require the following Lemma (cf. [MW1, p. 511]):

Lemma 2.2.2. Let $\pi: Y \rightarrow X$ be a finite and generically étale morphism of smooth proper and geometrically irreducible curves over a field $k$. If there is a geometric point of $X$ over which $\pi$ is totally ramified then the induced map of k-group schemes $\operatorname{Pic}(\pi): \operatorname{Pic}_{X / k} \rightarrow \operatorname{Pic}_{Y / k}$ has trivial schemetheoretic kernel.

Proof. We may assume that $k$ is algebraically closed. We fix a $k$-point $x \in X(k)$ over which $\pi$ is totally ramified, and let $y \in Y(k)$ be the unique $k$-point of $Y$ over $x$. To prove that $\operatorname{Pic}_{X / k} \rightarrow \operatorname{Pic}_{Y / k}$ has trivial kernel, it suffices to prove that the map of groups $\pi_{R}^{*}: \operatorname{Pic}\left(X_{R}\right) \rightarrow \operatorname{Pic}\left(Y_{R}\right)$ is injective for every $k$-algebra $R$. We fix such a $k$-algebra, and denote by $x_{R} \in X_{R}(R)$ and $y_{R} \in Y_{R}(R)$ the points obtained from $x$ and $y$ by base change. Let $\mathscr{L}$ be a line bundle on $X_{R}$ whose pullback to $Y_{R}$ is trivial; our claim is that we may choose a trivialization $\pi^{*} \mathscr{L} \stackrel{\simeq}{\rightarrow} \mathscr{O}_{Y_{R}}$ of $\pi^{*} \mathscr{L}$ over $Y_{R}$ which descends to $X_{R}$. In other words, by descent theory, we assert that we may choose a trivialization of $\pi^{*} \mathscr{L}$ with the property that the two pullback trivializations under the canonical projection maps

$$
Y_{R} \times_{X_{R}} Y_{R} \underset{\rho_{2}}{\stackrel{\rho_{1}}{\longrightarrow}} Y_{R}
$$

coincide.

We first claim that the $k$-scheme $Z:=Y \times_{X} Y$ is connected and generically reduced. Since $\pi$ is totally ramified over $x$, there is a unique geometric point $(y, y)$ of $Z$ mapping to $x$ under the canonical map $Z \rightarrow X$. Since this map is moreover finite flat (because $\pi: Y \rightarrow X$ is finite flat due to smoothness of $X$ and $Y)$, every connected component of $Z$ is finite flat onto $X$ and so passes through $(y, y)$. Thus, $Z$ is connected. On the other hand, $\pi: Y \rightarrow X$ is generically étale by hypothesis, so there exists a dense open subscheme $U \subseteq X$ over which $\pi$ is étale. Then $Z \times_{X} U$ is étale - and hence smooth-over $U$ and the open immersion $Z \times{ }_{X} U \rightarrow Z$ is schematically dense as $U \rightarrow X$ is schematically dense and $\pi$ is finite and flat. As $Z$ thus contains a $k$-smooth and dense subscheme, it is generically reduced.

Fix a choice $e$ of $R$-basis of the fiber $\mathscr{L}\left(x_{R}\right)$ of $\mathscr{L}$ at $x_{R}$. As any two trivializations of $\pi^{*} \mathscr{L}$ over $Y_{R}$ differ by an element of $R^{\times}$, we may uniquely choose a trivialization $\pi^{*} \mathscr{L} \simeq \mathscr{O}_{Y_{R}}$ with the property that the induced isomorphism

$$
\mathscr{L}\left(x_{R}\right) \simeq\left(\pi^{*} \mathscr{L}\right)\left(y_{R}\right) \stackrel{\simeq}{\longrightarrow} \mathscr{O}_{Y_{R}}\left(y_{R}\right) \simeq R
$$

carries $e$ to 1. The obstruction to the two pullback trivializations under (2.2.2) being equal is a global unit on $Y_{R} \times_{X_{R}} Y_{R}$. But since $Y_{R} \times_{X_{R}} Y_{R}=\left(Y \times_{X} Y\right)_{R}$, we have by flat base change

$$
H^{0}\left(Y_{R} \times_{X_{R}} Y_{R}, \mathscr{O}_{Y_{R} \times X_{R} Y_{R}}\right)=H^{0}\left(Y \times_{X} Y, \mathscr{O}_{Y \times_{X} Y}\right) \otimes_{k} R=R
$$

where the last equality rests on the fact that $Y \times_{X} Y$ is connected, generically reduced, and proper over $k$. Thus, the obstruction to the two pullback trivializations being equal is an element of $R^{\times}$, 
whose value may be calculated at any point of $Y_{R} \times_{X_{R}} Y_{R}$. By our choice (2.2.3) of trivialization of $\pi^{*} \mathscr{L}$, the value of this obstruction at the point $\left(y_{R}, y_{R}\right)$ is 1 , and hence the two pullback trivializations coincide as desired.

Proof of Proposition 2.2.1. Since $\rho: \operatorname{Ig}_{r} \rightarrow \operatorname{Ig}_{s}$ is a finite branched cover with group $\Delta_{s} / \Delta_{r}$ and totally wildly ramified over $\underline{\mathrm{S}}_{s}$, we may apply Proposition 2.1.7, which gives (1).

To prove (2), we work over $k:=\overline{\mathbf{F}}_{p}$ and argue as follows. Since $\rho: \operatorname{Ig}_{r} \rightarrow \operatorname{Ig}_{s}$ is of degree $p^{r-s}$ and totally ramified over $\underline{\mathrm{ss}}_{s}$, we have $\rho^{*} \underline{\mathrm{ss}}_{s}=p^{r-s} \cdot \underline{\mathrm{ss}}$; it follows that pullback induces a map

$$
H^{1}\left(\operatorname{Ig}_{s}, \mathscr{O}_{\mathrm{Ig}_{s}}\left(-\underline{\mathrm{SS}}_{s}\right)\right) \stackrel{\rho^{*}}{\longrightarrow} H^{1}\left(\operatorname{Ig}_{r}, \mathscr{O}_{\mathrm{Ig}_{r}}(-\underline{\mathrm{SS}})\right)
$$

which we claim is injective. To see this, we observe that the long exact cohomology sequence attached to the short exact sequence of sheaves on $\operatorname{Ig}_{r}$

$$
0 \longrightarrow \mathscr{O}_{\mathrm{Ig}_{r}}(-\underline{\mathrm{SS}}) \longrightarrow \mathscr{O}_{\mathrm{Ig}_{r}} \longrightarrow \mathscr{O}_{\underline{\mathrm{SS}}} \longrightarrow 0
$$

(with $\mathscr{O}_{\underline{s}}$ a skyscraper sheaf supported on $\underline{\mathrm{ss}}$ ) yields a commutative diagram with exact rows

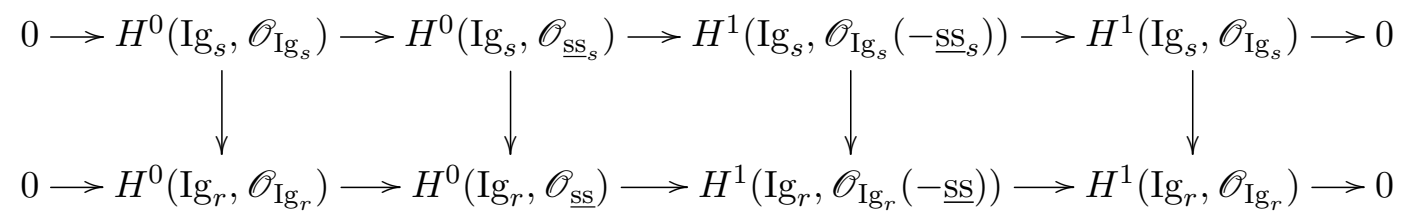

The leftmost vertical arrow is an isomorphism because $\mathrm{Ig}_{r}$ is geometrically connected for all $r$. Since $\underline{\text { ss }}$ is reduced, we have $H^{0}\left(\operatorname{Ig}_{r}, \mathscr{O}_{\underline{\mathrm{SS}}}\right)=k^{\mathrm{deg}} \underline{\underline{\mathrm{SS}}}$ for all $r$, so since $\rho: \operatorname{Ig}_{r} \rightarrow \operatorname{Ig}_{s}$ totally ramifies over every supersingular point, the second vertical arrow in (2.2.5) is also an isomorphism. Now the rightmost vertical map in (2.2.5) is identified with the map on Lie algebras $\mathrm{Lie} \mathrm{Pic}_{\mathrm{Ig}_{s} / k}^{0} \rightarrow \mathrm{Lie} \mathrm{Pic}_{\mathrm{Ig}_{r} / k}^{0}$ induced by $\operatorname{Pic}^{0}(\rho)$, which is injective thanks to Lemma 2.2.2 and the left-exactness of the functor Lie. An easy diagram chase using (2.2.5) then shows that (2.2.4) is injective, as claimed.

Using again the equality $\rho^{*}\left(\underline{\mathbf{S}}_{s}\right)=p^{r-s} \cdot \underline{\mathrm{s}}$, pullback of meromorphic differentials yields a mapping

$$
H^{0}\left(\operatorname{Ig}_{s}, \Omega_{\operatorname{Ig}_{s} / k}^{1}\left(\underline{\mathrm{ss}}_{s}\right)\right) \longrightarrow H^{0}\left(\operatorname{Ig}_{r}, \Omega_{\operatorname{Ig}_{r} / k}^{1}\left(p^{r-s} \cdot \underline{\mathrm{s}}\right)\right)
$$

which is injective since $\rho: \operatorname{Ig}_{r} \rightarrow \operatorname{Ig}_{s}$ is separable.

Dualizing the injective maps (2.2.4) and (2.2.6), we see that the canonical trace mappings

$$
\begin{gathered}
H^{0}\left(\operatorname{Ig}_{r}, \Omega_{\operatorname{Ig}_{r} / k}^{1}(\underline{\mathrm{ss}})\right) \stackrel{\rho_{*}}{\longrightarrow} H^{0}\left(\operatorname{Ig}_{s}, \Omega_{\operatorname{Ig}_{s} / k}^{1}\left(\underline{\mathrm{ss}}_{s}\right)\right) \\
H^{1}\left(\operatorname{Ig}_{r}, \mathscr{O}_{\operatorname{Ig}_{r}}\left(-p^{r-s} \cdot \underline{\mathrm{ss}}\right)\right) \stackrel{\rho_{*}}{\longrightarrow} H^{1}\left(\operatorname{Ig}_{s}, \mathscr{O}_{\operatorname{Ig}_{s}}\left(-\underline{\mathrm{SS}}_{s}\right)\right)
\end{gathered}
$$

are surjective for all $r \geq s \geq 1$. Passing to $V$ - (respectively $F$-) ordinary parts and using Lemma 2.1.6 (1), we conclude that the canonical trace mappings attached to $\operatorname{Ig}_{r} \rightarrow \operatorname{Ig}_{s}$ induce surjective maps as in Proposition 2.2.1 (2). By (1), these mappings are then surjective mappings of free $\mathbf{F}_{p}\left[\Delta_{1} / \Delta_{s}\right]$-modules of the same rank, and are hence isomorphisms.

The group $\mathbf{F}_{p}^{\times}$acts naturally on the Igusa curve $\operatorname{Ig}_{1}$ through the diamond operators (see below Definition B.12), and the eigenspaces for the induced action on $H^{0}\left(\operatorname{Ig}_{1}, \Omega_{\operatorname{Ig}_{1} / \mathbf{F}_{p}}^{1}(\underline{\mathrm{ss}})\right)$ are intimately connected with mod $p$ cusp forms: 
Proposition 2.2.3. Let $S_{k}\left(\Gamma_{1}(N) ; \mathbf{F}_{p}\right)$ be the space of weight $k$ cuspforms for $\Gamma_{1}(N)$ over $\mathbf{F}_{p}$, and for $0 \leq j<p$ denote by $\left.H^{0}\left(\operatorname{Ig}_{1}, \Omega_{\operatorname{Ig}_{1} / \mathbf{F}_{p}}^{1} \underline{(\underline{\mathrm{s}})}\right)\right)^{(j)}$ the subspace of $H^{0}\left(\operatorname{Ig}_{1}, \Omega_{\operatorname{Ig}_{1} / \mathbf{F}_{p}}^{1}(\underline{\mathrm{ss}})\right)$ on which $\mathbf{F}_{p}^{\times}$acts through the $j$-th power of the Teichmüller character. For each $k$ with $2<k<p+1$, there are canonical isomorphisms of $\mathbf{F}_{p}$-vector spaces

$$
S_{k}\left(\Gamma_{1}(N) ; \mathbf{F}_{p}\right) \simeq H^{0}\left(\operatorname{Ig}_{1}, \Omega_{\operatorname{Ig}_{1} / \mathbf{F}_{p}}^{1}\right)^{(k-2)} \simeq H^{0}\left(\operatorname{Ig}_{1}, \Omega_{\operatorname{Ig}_{1} / \mathbf{F}_{p}}^{1}(\underline{\mathrm{ss}})\right)^{(k-2)}
$$

which are equivariant for the Hecke operators and diamond operators $\langle\cdot\rangle_{N}$, with $U_{p}$ acting as usual on modular forms and as the Cartier operator $V$ on differential forms. For $k=2, p+1$, we have the following commutative diagram:

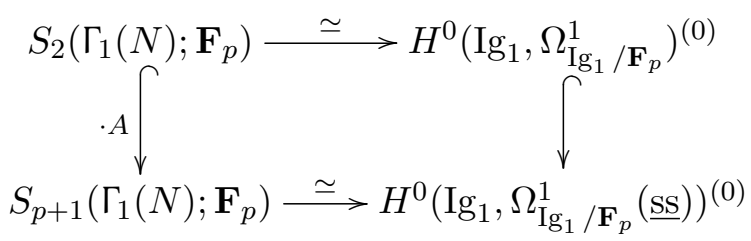

where $A$ is the Hasse invariant.

Proof. This follows from Propositions 5.7-5.10 of [Gross], using Lemma 2.3.9 (keeping in mind Remark B.13); we note that our forward reference to Lemma 2.3.9 does not result in circular reasoning.

Remark 2.2.4. For each $k$ with $2 \leq k \leq p+1$, let us write $d_{k}:=\operatorname{dim}_{\mathbf{F}_{p}} S_{k}\left(\Gamma_{1}(N) ; \mathbf{F}_{p}\right)^{\text {ord }}$ for the $\mathbf{F}_{p^{-}}$ dimension of the subspace of weight $k$ level $N$ cuspforms over $\mathbf{F}_{p}$ on which ${ }^{5} U_{p}:=T_{p}$ acts invertibly.

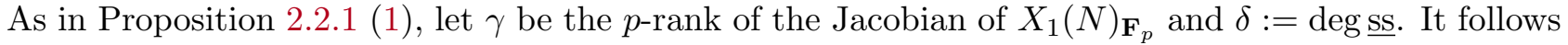
immediately from Proposition 2.2.3 that we have the equality

$$
d:=\gamma+\delta-1=\sum_{k=3}^{p+1} d_{k} .
$$

2.3. Structure of the ordinary part of $H^{0}\left(\bar{X}_{r}, \omega_{\bar{X}_{r} / \mathbf{F}_{p}}\right)$. As in the introduction to $\S 2$, we write $X_{r}$ for the regular proper model of $X_{1}\left(N p^{r}\right)$ over $R_{r}$ given by Definition B.6, and we denote by $\bar{X}_{r}:=X_{r} \times{ }_{R_{r}} \mathbf{F}_{p}$ the special fiber of $X_{r}$. There is a natural "semilinear" action of $\Gamma=\operatorname{Gal}\left(\mathbf{Q}_{p}\left(\mu_{p} \infty\right) / \mathbf{Q}_{p}\right)$ on the scheme $X_{r}$ given by (B.3), which records the fact that its generic fiber is defined over $\mathbf{Q}_{p}$; this action induces a "geometric inertia action" of $\Gamma$ on the special fiber $\bar{X}_{r}$ over $\mathbf{F}_{p}$.

By Proposition B.14, the curve $\bar{X}_{r}$ is a "disjoint union with crossings at the supersingular points" of Igusa curves $I_{(a, b, u)}:=\operatorname{Ig}_{\max (a, b)}$ indexed by triples $(a, b, u)$ with $a, b$ nonnegative integers satisfying $a+b=r$ and $u$ ranging over all elements of $\left(\mathbf{Z} / p^{\min (a, b)} \mathbf{Z}\right)^{\times}$. Using Rosenlicht's explicit description of the relative dualizing sheaf $\omega_{\bar{x}_{r} / \mathbf{F}_{p}}$ as a certain sheaf of meromorphic differentials on the normalization $\bar{x}_{r}^{\mathrm{n}}$ of $\bar{X}_{r}$, we now relate the ordinary part of the cohomology $H\left(\bar{X}_{r} / \mathbf{F}_{p}\right)$ given by (2.0.1) to the de Rham cohomology of the Igusa tower.

As in Remark B.16, we write $I_{r}^{\infty}:=I_{(r, 0,1)}$ and $I_{r}^{0}:=I_{(0, r, 1)}$ for the two "good" components of $\bar{X}_{r}$, each of which is a copy of $\operatorname{Ig}_{r}$. For $\star=0, \infty$, we denote by $i_{r}^{\star}: I_{r}^{\star} \hookrightarrow \bar{X}_{r}$ the canonical closed immersion, and for $s \leq r$, we write simply $\rho: I_{r}^{\star} \rightarrow I_{s}^{\star}$ for the the degeneracy map (B.7) on Igusa curves. To ease the notational burden in what follows, we will frequently omit subscrips on sheaves when they are

\footnotetext{
${ }^{5}$ In this characteristic $p$ setting, it is standard to write $U_{p}$ for the Hecke operator $T_{p}$, as the effect of these operators on $q$-expansions agrees in characteristic $p$; cf. [Gross, §4].
} 
clear from context and write, for example, $\omega_{\bar{x}_{r}}$ (respectively $\Omega_{I_{r}^{\star}}^{1}$ ) or even just $\omega$ (resp. $\Omega^{1}$ ) in place of $\omega_{\bar{x}_{r} / \mathbf{F}_{p}}\left(\right.$ resp. $\left.\Omega_{I_{r}^{\star} / \mathbf{F}_{p}}^{1}\right)$, and $\mathscr{O}$ in place of $\mathscr{O}_{I_{r}^{\star}}$ or $\mathscr{O}_{\bar{x}_{r}}$.

The diamond operator and Hecke correspondences on $X_{r}$ and $I_{r}^{\star} \simeq \operatorname{Ig}_{r}$ are defined in Appendix B (see especially Definition B.26). Each of these correspondences $T$ induces endomorphisms $T$ and $T^{*}$ of $H^{0}\left(\bar{X}_{r}, \omega_{\bar{X}_{r} / \mathbf{F}_{p}}\right)$ via Lemma A.14 (1) and (A.11) (see below), and of $H^{0}\left(I_{r}^{\star}, \Omega_{I_{r}^{\star} / \mathbf{F}_{p}}^{1}(\underline{\mathbf{s}})\right)$ via (A.11). There are thus two possible ways of viewing each of these cohomology groups as modules over the group ring $\mathbf{F}_{p}\left[\Delta / \Delta_{r}\right]$ of diamond operators at $p$ : either via the "usual" action, or the adjoint action. We give $e_{r}^{*} H^{0}\left(\bar{X}_{r}, \omega_{\bar{X}_{r} / \mathbf{F}_{p}}\right)$ (respectively $e_{r} H^{0}\left(\bar{X}_{r}, \omega_{\bar{X}_{r} / \mathbf{F}_{p}}\right)$ ) the structure induced by the adjoint (respectively usual) diamond operator action, which induces the same $\mathbf{F}_{p}\left[\Delta_{1} / \Delta_{r}\right]$-module structure arising from the natural $\mathfrak{H}_{r}^{*}\left(\right.$ respectively $\mathfrak{H}_{r}$ ) module structure and the $\Lambda$-algebra structure of $\mathfrak{H}^{*}$ (respectively $\mathfrak{H}$ ) fixed in $\S 1.4$. On the other hand, in what follows we will always give $H^{0}\left(I_{r}^{\star}, \Omega_{I_{r}^{\star} / \mathbf{F}_{p}}^{1}(\underline{\mathrm{ss}})\right)$ the $\mathbf{F}_{p}\left[\Delta / \Delta_{r}\right]-$ module structure determined by requiring that $u \in \Delta$ act as $\langle u\rangle$ (and not the adjoint $\langle u\rangle^{*}=\left\langle u^{-1}\right\rangle$ ).

Proposition 2.3.1. For each positive integer $r$, pullback of differentials along $i_{r}^{0}$ (respectively $i_{r}^{\infty}$ ) induces a natural isomorphism of $\mathbf{F}_{p}\left[\Delta / \Delta_{r}\right]$-modules

$$
e_{r}^{*} H^{0}\left(\bar{X}_{r}, \omega_{\bar{X}_{r}}\right) \underset{\left(i_{r}^{0}\right)^{*}}{\simeq} H^{0}\left(I_{r}^{0}, \Omega^{1}(\underline{\mathrm{ss}})\right)^{V_{\text {ord }}}, \text { resp. } e_{r} H^{0}\left(\bar{X}_{r}, \omega_{\bar{x}_{r}}\right) \underset{\left(i_{r}^{\infty}\right)^{*}}{\simeq} H^{0}\left(I_{r}^{\infty}, \Omega^{1}(\underline{\mathrm{ss}})\right)^{V_{\text {ord }}} .
$$

that is equivariant for the Hecke operators $T_{\ell}^{*}, U_{\ell}^{*}$ and $\langle v\rangle_{N}^{*}\left(\right.$ respectively $T_{\ell}, U_{\ell}$ and $\left.\langle v\rangle_{N}\right)$ and $\Gamma$ equivariant for the geometric inertia action on $\bar{X}_{r}$ and the action $\gamma \mapsto\left\langle\chi(\gamma)^{-1}\right\rangle^{*}$ (respectively the trivial action) on $H^{0}\left(I_{r}^{\star}, \Omega_{I_{r}^{\star}}^{1}(\underline{\mathrm{ss}})\right)$ for $\star=0$ (resp. $\left.\star=\infty\right)$. The isomorphisms (2.3.1) induce identifications that are compatible with change in $r$ : the diagrams formed by taking the interior or the exterior arrows
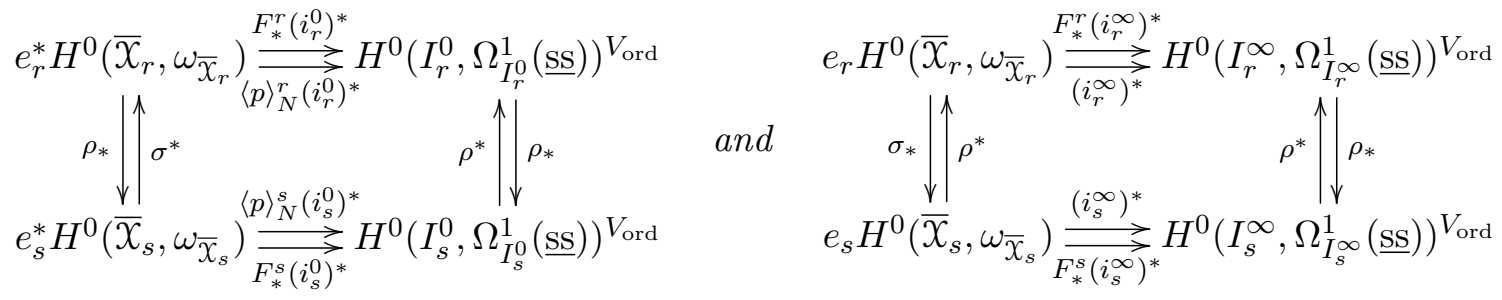

are all commutative for $s \leq r$. The same assertions hold true if we replace $\bar{X}_{*}$ with $\bar{X}_{*}^{\mathrm{n}}$ and $\Omega_{I_{*}^{\star}}^{1}(\underline{\mathrm{ss}})$ with $\Omega_{I_{*}^{\star}}^{1}$ throughout.

We will prove Proposition 2.3.1 via a series of Lemmas. To proceed with the proof, we first fix some notation and ideas. To begin with, we may and do work over $k:=\overline{\mathbf{F}}_{p}$. If $X$ is any $\mathbf{F}_{p^{-}}$-scheme, we will abuse notation slightly by again writing $X$ for the base change of $X$ to $k$, and we write $F: X \rightarrow X$ for the base change of the absolute Frobenius of $X$ over $\mathbf{F}_{p}$ to $k$.

Let $\bar{X}_{r}^{\mathrm{n}} \rightarrow \bar{X}_{r}$ be the normalization map; by Proposition B.14, we know that $\bar{X}_{r}^{\mathrm{n}}$ is the disjoint union of proper smooth and irreducible Igusa curves $I_{(a, b, u)}=\operatorname{Ig}_{\max (a, b)}$ indexed by triples $(a, b, u)$ with $a, b$ nonnegative integers satisfying $a+b=r$ and $u \in\left(\mathbf{Z} / p^{\min (a, b)} \mathbf{Z}\right)^{\times}$. Rosenlicht's explicit description of the relative dualizing sheaf (see Proposition A.13) provides a functorial injection of $k$-vector spaces

$$
H^{0}\left(\bar{X}_{r}, \omega_{\bar{x}_{r}}\right) \longleftrightarrow H^{0}\left(\bar{x}_{r}^{\mathrm{n}}, \Omega_{k\left(\bar{x}_{r}^{\mathrm{n}}\right)}^{1}\right) \simeq \prod_{(a, b, u)} \Omega_{k\left(I_{(a, b, u)}\right)}^{1}
$$

with image precisely those elements $\left(\eta_{(a, b, u)}\right)$ of the product that are holomorphic outside the supersingular points and satisfy $\sum \operatorname{res}_{x_{(a, b, u)}}\left(s \eta_{(a, b, u)}\right)=0$ for each supersingular point $x \in \bar{X}_{r}(k)$ and all 
$s \in \mathscr{O}_{\bar{x}_{r}, x}$, where $x_{(a, b, u)}$ is the unique point of $I_{(a, b, u)}$ lying over $x$ and the sum is over all triples $(a, b, u)$ as above. We henceforth identify $H^{0}\left(\bar{X}_{r}, \omega_{\bar{x}_{r}}\right)$ with a subspace of the space of meromorphic differential forms on $\bar{X}_{r}^{\mathrm{n}}$ via $(2.3 .3)$, and for any meromorphic differential $\eta$, we denote by $\eta_{(a, b, u)}$ the $(a, b, u)$-component of $\eta$.

The correspondence $U_{p}:=\left(\pi_{1}, \pi_{2}\right)$ on $X_{r}$ is given by the degeneracy maps $\pi_{1}, \pi_{2}: y_{r} \rightrightarrows X_{r}$ of (B.9) and yields, as in (A.11), endomorphisms $U_{p}:=\left(\pi_{1}\right)_{*} \circ \pi_{2}^{*}$ and $U_{p}^{*}:=\left(\pi_{2}\right)_{*} \circ \pi_{1}^{*}$ of $H^{0}\left(X_{r}, \omega_{X_{r} / R_{r}}\right)$; we will again denote by $U_{p}$ and $U_{p}^{*}$ the induced endomorphisms $U_{p} \otimes 1$ and $U_{p}^{*} \otimes 1$ of

$$
H^{0}\left(\bar{X}_{r}, \omega_{\bar{X}_{r} / k}\right) \simeq H^{0}\left(\mathcal{X}_{r}, \omega_{X_{r} / R_{r}}\right) \otimes_{R_{r}} k,
$$

where the isomorphism is the canonical one of Lemma A.14 (1). By the functoriality of normalization, we have an induced correspondence $U_{p}:=\left(\bar{\pi}_{1}^{\mathrm{n}}, \bar{\pi}_{2}^{\mathrm{n}}\right)$ on $\bar{X}_{r}^{\mathrm{n}}$, and we write $U_{p}$ and $U_{p}^{*}$ for the resulting endomorphisms (A.11) of $H^{0}\left(\bar{X}_{r}^{\mathrm{n}}, \underline{\Omega}_{k\left(\bar{X}_{r}^{\mathrm{n}}\right)}^{1}\right)$. By Lemma A.14 (2), the map (2.3.3) is then $U_{p}$ and $U_{p^{-}}^{*}$ equivariant. The Hecke correspondences away from $p$ and the diamond operators act on the source of (2.3.3) via "reduction modulo $p$ " and on the target via the induced correspondences in the usual way (A.11), and the map (2.3.3) is compatible with these actions thanks to Lemma A.14 (2). Similarly, the semilinear "geometric inertia" action of $\Gamma$ on $X_{r}$ induces a linear action on $\bar{X}_{r}^{\mathrm{n}}$ as in Proposition B.18, and the map (2.3.3) is equivariant with respect to these actions.

With these preliminaries out of the way, our first task is to describe the action of $U_{p}$ and $U_{p}^{*}$ on the space of meromorphic differentials on $\bar{X}_{r}^{\mathrm{n}}$ :

Lemma 2.3.2. For any meromorphic differential $\eta=\left(\eta_{(a, b, u)}\right)$ on $\bar{x}_{r}^{\mathrm{n}}$, we have

$$
\left(U_{p} \eta\right)_{(a, b, u)}=\left\{\begin{array}{lll}
F_{*} \eta_{(r, 0,1)} & : \quad(a, b, u)=(r, 0,1) \\
\rho_{*} \eta_{(a+1, b-1, u)} & : \quad 0<b \leq a \\
\sum_{u^{\prime} \in\left(\mathbf{Z} / p^{a+1} \mathbf{Z}\right)^{\times}}\left\langle u^{\prime}\right\rangle \eta_{(a+1, b-1, u)} & : \quad r \text { odd }, a=b-1 \\
u^{\prime} \equiv u \bmod p^{a} & \\
\sum_{u^{\prime} \in\left(\mathbf{Z} / p^{a+1} \mathbf{Z}\right)^{\times}} \rho^{*}\left\langle u^{\prime}\right\rangle \eta_{\left(a+1, b-1, u^{\prime}\right)} & : \quad r \text { even }, a=b-2 \\
u^{\prime} \equiv u \bmod p^{a} & \\
\sum_{u^{\prime} \in\left(\mathbf{Z} / p^{a+1} \mathbf{Z}\right)^{\times}} \rho^{*} \eta_{\left(a+1, b-1, u^{\prime}\right)} & : \quad 0 \leq a<b-2 \\
u^{\prime} \equiv u \bmod p^{a} &
\end{array}\right.
$$

and

$$
\left(U_{p}^{*} \eta\right)_{(a, b, u)}=\left\{\begin{array}{lll}
\langle p\rangle_{N}^{-1} F_{*} \eta_{(0, r, 1)} & : \quad(a, b, u)=(0, r, 1) \\
\rho_{*} \eta_{(a-1, b+1, u)} & : \quad 0<a<b \\
\langle u\rangle^{-1} \rho_{*} \eta_{(a-1, b+1, u)} & : \quad r \text { even }, b=a \\
\sum_{u^{\prime} \in\left(\mathbf{Z} / p^{b+1} \mathbf{Z}\right)^{\times}}\left\langle u^{\prime}\right\rangle^{-1} \eta_{\left(a-1, b+1, u^{\prime}\right)} & : \quad r \text { odd }, b=a-1 \\
u^{\prime} \equiv u \bmod p^{b} & \\
\sum_{u^{\prime} \in\left(\mathbf{Z} / p^{b+1} \mathbf{Z}\right)^{\times}} \rho^{*} \eta_{\left(a-1, b+1, u^{\prime}\right)} & : \quad 0 \leq b<a-1 \\
u^{\prime} \equiv u \bmod p^{b} &
\end{array}\right.
$$


Proof. This is an easy exercise using the definition of $U_{p}$, the explicit description of the maps $\bar{\pi}_{1}^{\mathrm{n}}$ and $\bar{\pi}_{2}^{\mathrm{n}}$ given in Proposition B.25, and the fact that $F^{*}$ kills any global meromorphic differential form on a scheme of characteristic $p$.

Observe that for $0<b \leq r$, the $(a, b, u)$-component of $U_{p} \eta$ depends only on the $\left(a+1, b-1, u^{\prime}\right)$ components of $\eta$ for varying $u^{\prime}$, and similarly for $0<a \leq r$ the $(a, b, u)$-component of $U_{p}^{*} \eta$ depends only on the $\left(a-1, b+1, u^{\prime}\right)$-components of $\eta$. This fact is crucial for our purposes, and manifests itself clearly in the following:

Corollary 2.3.3. For any $n \geq r \geq 1$, and any meromorphic differential $\eta=\left(\eta_{(a, b, u)}\right)$ on $\bar{x}_{r}^{\mathrm{n}}$, we have

$$
\left(U_{p}^{n} \eta\right)_{(a, b, u)}=\left\{\begin{array}{lll}
\rho_{*}^{b} F_{*}^{n-b} \eta_{(r, 0,1)} & : \quad b \leq a \\
\sum_{u^{\prime} \in\left(\mathbf{Z} / p^{b} \mathbf{Z}\right)^{\times}}\left\langle u^{\prime}\right\rangle \rho_{*}^{a} F_{*}^{n-b} \eta_{(r, 0,1)} & : \quad a<b \\
u^{\prime} \equiv u \bmod p^{a} & &
\end{array}\right.
$$

and

$$
\left(U_{p}^{* n} \eta\right)_{(a, b, u)}= \begin{cases}\rho_{*}^{a}\langle p\rangle_{N}^{a-n} F_{*}^{n-a} \eta_{(0, r, 1)} & : \quad a<b \\ \sum_{u^{\prime} \in\left(\mathbf{Z} / p^{a} \mathbf{Z}\right)^{\times}}\left\langle u^{\prime}\right\rangle^{-1} \rho_{*}^{b}\langle p\rangle_{N}^{a-n} F_{*}^{n-a} \eta_{(0, r, 1)} & : \quad b \leq a \\ u^{\prime} \equiv u \bmod p^{b} & \end{cases}
$$

Proof. This follows immediately from Lemma 2.3.2 by induction.

Lemma 2.3.4. Pullback of meromorphic differential forms along $i_{r}^{\star}: I_{r}^{\star} \hookrightarrow \bar{X}_{r}^{\mathrm{n}}$ for $\star=\infty$ (respectively $\star=0)$ carries $e_{r} H^{0}\left(\bar{X}_{r}, \omega\right)$ (respectively $\left.e_{r}^{*} H^{0}\left(\bar{X}_{r}, \omega\right)\right)$ into $H^{0}\left(I_{r}^{\star}, \Omega^{1}(\underline{\mathrm{s}})\right)^{V_{\text {ord }}}$. Moreover, the resulting maps (2.3.1) intertwine $U_{p}$ (respectively $\left.U_{p}^{*}\right)$ on source with $F_{*}$ (respectively $\left.\langle p\rangle_{N}^{-1} F_{*}\right)$ on target.

Proof. We will treat the case of $\star=\infty$; the case $\star=0$ is similar. We first observe that pullback of meromorphic differentials along $i_{r}^{\infty}: I_{r}^{\infty} \hookrightarrow \bar{X}_{r}^{\mathrm{n}}$ is given by projection

$$
H^{0}\left(\bar{X}_{r}^{\mathrm{n}}, \underline{\Omega}_{k\left(\bar{X}_{r}^{\mathrm{n}}\right)}^{1}\right) \simeq \prod_{(a, b, u)} H^{0}\left(I_{(a, b, u)}, \underline{\Omega}_{k\left(I_{(a, b, u)}\right)}^{1} \stackrel{\operatorname{proj}_{(r, 0,1)}}{\longrightarrow} H^{0}\left(I_{r}^{\infty}, \underline{\Omega}_{k\left(I_{r}^{\infty}\right)}^{1}\right)\right.
$$

onto the $(r, 0,1)$-component. Thanks to Rosenlicht's description of the image of (2.3.3), any section $\eta=\left(\eta_{(a, b, u)}\right)$ of $H^{0}\left(\bar{X}_{r}, \omega_{\bar{x}_{r}}\right)$ has the property that $\eta_{(a, b, u)}$ is holomorphic outside $\underline{\text { s }}$ and, due to [Conrad, Lemma 5.2.2], has poles along ss of order bounded by a constant $N_{r}$ depending only on $r$. Thus, the composition of pullback along $i_{r}^{\infty}$ with (2.3.3) induces a map

$$
\left(i_{r}^{\infty}\right)^{*}: e_{r} H^{0}\left(\bar{X}_{r}, \omega_{\bar{X}_{r}}\right) \longrightarrow H^{0}\left(I_{r}^{\infty}, \Omega_{I_{r}^{\infty}}^{1}\left(N_{r} \cdot \underline{\mathrm{ss}}\right)\right)
$$

which moreover satisfies $\left(i_{r}^{\infty}\right)^{*} \circ U_{p}=F_{*} \circ\left(i_{r}^{\infty}\right)^{*}$ thanks to the description (2.3.4a) of Lemma 2.3.2. Since $U_{p}$ is invertible on the source of $(2.3 .7)$, it follows at once from this intertwining relation that $F_{*}$ is invertible on its image, and we conclude from Lemma 2.1.6 (1) that (2.3.7), has image contained in $H^{0}\left(I_{r}^{\infty}, \Omega_{I_{r}^{\infty}}^{1}(\underline{\mathrm{ss}})\right)^{V_{\text {ord }}}$, as claimed.

In order to prove that the pullback maps (2.3.1) resulting from Lemma 2.3.4 are isomorphisms, we will construct the inverse mappings. For any $r>0$ and for $\star=\infty, 0$ we now define maps

$$
\gamma_{r}^{\star}: H^{0}\left(I_{r}^{\star}, \Omega_{I_{r}^{\star}}^{1}(\underline{\mathrm{s}})\right)^{V_{\text {ord }}} \longrightarrow H^{0}\left(\bar{X}_{r}^{\mathrm{n}}, \underline{\Omega}_{k\left(\bar{X}_{r}^{\mathrm{n}}\right)}^{1}\right)
$$


by

$$
\left(\gamma_{r}^{\infty}(\nu)\right)_{(a, b, u)}:= \begin{cases}\rho_{*}^{b} F_{*}^{-b} \nu & : \quad b \leq a \\ \sum_{u^{\prime} \in\left(\mathbf{Z} / p^{b} \mathbf{Z}\right)^{\times}}\left\langle u^{\prime}\right\rangle \rho_{*}^{a} F_{*}^{-b} \nu & : \quad a<b \\ u^{\prime} \equiv u \bmod p^{a} & \end{cases}
$$

and

$$
\left(\gamma_{r}^{0}(\nu)\right)_{(a, b, u)}:=\left\{\begin{array}{lll}
\rho_{*}^{a}\langle p\rangle_{N}^{a} F_{*}^{-a} \nu & : \quad a<b \\
\sum_{u^{\prime} \in\left(\mathbf{Z} / p^{a} \mathbf{Z}\right)^{\times}}\left\langle u^{\prime}\right\rangle^{-1} \rho_{*}^{b}\langle p\rangle_{N}^{a} F_{*}^{-a} \nu_{(0, r, 1)} & : \quad b \leq a \\
u^{\prime} \equiv u \bmod p^{b} & &
\end{array}\right.
$$

These maps are well-defined because $F_{*}=V$ is invertible on the $V$-ordinary subspace.

Lemma 2.3.5. The maps $\gamma_{r}^{\star}$ satisfy $U_{p} \circ \gamma_{r}^{\infty}=\gamma_{r}^{\infty} \circ F_{*}$ and $U_{p}^{*} \circ \gamma_{r}^{0}=\gamma_{r}^{0} \circ\langle p\rangle_{N}^{-1} F_{*}$. Via (2.3.3), the map $\gamma_{r}^{\infty}$ (respectively $\left.\gamma_{r}^{0}\right)$ has image contained in $e_{r} H^{0}\left(\bar{X}_{r}, \omega\right)$ (respectively $e_{r}^{*} H^{0}\left(\bar{X}_{r}, \omega\right)$ ).

Proof. The asserted intertwining relations follow readily from Lemma 2.3.2 and the definitions (2.3.8a)(2.3.8b). Since $F_{*}$ is invertible on the source of $\gamma_{r}^{\star}$, to complete the proof it then suffices to show that $\gamma_{r}^{\star}$ has image in $H^{0}\left(\bar{X}_{r}, \omega_{\bar{x}_{r} / k}\right)$.

To do this, we again appeal to the explicit description of the image of (2.3.3) afforded by Rosenlicht's theory, and must check that for any supersingular point $x$ on $\bar{x}_{r}$, and any local section $s \in \mathscr{O}_{\bar{x}_{r}, x}$, the sum of the residues of $s \gamma^{\star}(\nu)$ at all $k$-points of $\bar{X}_{r}^{\mathrm{n}}$ lying over $x$ is zero. Using (2.3.8a), we calculate that for $\star=\infty$ this sum is equal to

$$
\sum_{b \leq a} \sum_{u \in\left(\mathbf{Z} / p^{b} \mathbf{Z}\right)^{\times}} \operatorname{res}_{x_{(a, b, u)}}\left(s \rho_{*}^{b} F_{*}^{-b} \nu\right)+\sum_{a<b} \sum_{u \in\left(\mathbf{Z} / p^{b} \mathbf{Z}\right)^{\times}} \operatorname{res}_{x_{(a, b, u)}}\left(s\langle u\rangle \rho_{*}^{a} F_{*}^{-b} \nu\right)
$$

where $x_{(a, b, u)}$ denotes the unique point of the $(a, b, u)$-component of $\bar{X}_{r}^{\mathrm{n}}$ over $x$, and the outer sums range over all nonnegative integers $a, b$ with $a+b=r$. We claim that for any meromorphic differential $\omega$ on $I_{(a, b, u)}$ and any supersingular point $y$ of $I_{(a, b, u)}$ over $x$, we have

$$
\operatorname{res}_{y}(\omega)=\operatorname{res}_{y}(\langle v\rangle \omega)
$$

for all $v \in \mathbf{Z}_{p}^{\times}$, and, if in addition $\omega$ is $V$-ordinary,

$$
\operatorname{res}_{y}(s \omega)=s(x) \operatorname{res}_{y}(\omega)
$$

Indeed, (2.3.10a) is a consequence of (2.1.4), using the fact that the automorphism $\langle v\rangle$ of $I_{(a, b, u)}$ fixes every supersingular point, while $(2.3 .10 \mathrm{~b})$ is deduced by thinking about formal expansions of differentials at $y$ (cf. Proposition 2.1.1 (4) and (5)) and using the fact that a $V$-ordinary meromorphic differential has at worst simple poles by Lemma 2.1.6. Via (2.3.10a)-(2.3.10b), we reduce (2.3.9) to

$$
\begin{aligned}
\sum_{a+b=r} \sum_{u \in\left(\mathbf{Z} / p^{b} \mathbf{Z}\right)^{\times}} s(x) \operatorname{res}_{x_{(a, b, u)}}\left(\rho_{*}^{\min (a, b)} F_{*}^{-b} \nu\right) & =\sum_{a+b=r} \varphi\left(p^{b}\right) s(x) \operatorname{res}_{x_{(a, b, 1)}}\left(\rho_{*}^{\min (a, b)} F_{*}^{-b} \nu\right) \\
& =s(x) \operatorname{res}_{x_{(r, 0,1)}}(\nu)-s(x) \operatorname{res}_{x_{(r, 0,1)}}\left(F_{*}^{-1} \nu\right)
\end{aligned}
$$

where the first equality above follows from the fact that for fixed $a, b$, the points $x_{(a, b, u)}$ for varying $u \in\left(\mathbf{Z} / p^{\min (a, b)} \mathbf{Z}\right)^{\times}$are all identified with the same point on $\operatorname{Ig}\left(p^{\max (a, b)}\right)$, and the second equality is a consequence of (2.1.4), which gives $\operatorname{res}_{x_{(r-1,1,1)}}\left(\rho_{*} F_{*}^{-1} \nu\right)=\operatorname{res}_{x_{(r, 0,1)}}\left(F_{*}^{-1} \nu\right)$ as $\rho: I_{(r, 0,1)} \rightarrow I_{(r-1,1,1)}$ is generically étale and carries the point $x_{(r, 0,1)}$ to $x_{(r-1,1,1)}$. As $\nu$ is $V$-ordinary, there exists a $V$-ordinary 
meromorphic differential $\xi$ on $I_{r}^{\infty}$ with $\nu=F_{*} \xi$; substituting this expression for $\nu$ into (2.3.11) and applying Proposition 2.1.1 (5), we conclude that (2.3.11) is zero, as desired. That $\gamma_{r}^{0}$ has image in $H^{0}\left(\bar{X}_{r}, \omega_{\bar{X}_{r} / k}\right)$ follows from a nearly identical calculation, and we omit the details.

From Lemmas 2.3.4 and 2.3.5 we thus obtain natural maps

$$
H^{0}\left(I_{r}^{\infty}, \Omega_{I_{r}^{\infty} / k}^{1}(\underline{\mathrm{s}})\right)^{V_{\text {ord }}} \underset{\left(i_{r}^{\infty}\right)^{*}}{\stackrel{\gamma_{r}^{\infty}}{\longrightarrow}} e_{r} H^{0}\left(\overline{\mathcal{X}}_{r}, \omega_{\bar{X}_{r} / k}\right)
$$

and

$$
H^{0}\left(I_{r}^{0}, \Omega_{I_{r}^{0} / k}^{1}(\underline{\mathrm{ss}})\right)^{V_{\text {ord }}} \underset{\left(i_{r}^{0}\right)^{*}}{\stackrel{\gamma_{r}^{0}}{\longrightarrow}} e_{r}^{*} H^{0}\left(\overline{\mathcal{X}}_{r}, \omega_{\overline{\mathcal{X}}_{r} / k}\right)
$$

intertwining $F_{*}$ and $\langle p\rangle_{N}^{-1} F_{*}$ on the left with $U_{p}$ and $U_{p}^{*}$ on the right, respectively.

Lemma 2.3.6. The maps (2.3.12a) and (2.3.12b) are inverse isomorphisms.

Proof. From the very definitions (2.3.8a) of $\gamma_{r}^{\infty}$ and the description (2.3.6) of pullback as projection, it

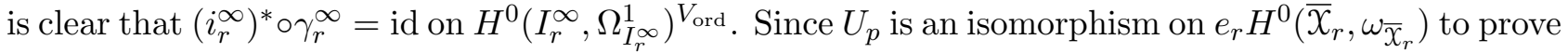
that the composition in the other order is the identity, it suffices to prove that $U_{p}^{n} \circ \gamma_{r}^{\infty} \circ\left(i_{r}^{\infty}\right)^{*}=U_{p}^{n}$ on $e_{r} H^{0}\left(\bar{X}_{r}, \omega_{\bar{X}_{r}}\right)$ for any $n>r$. Using the intertwining relations established by Lemmas 2.3 .4 and 2.3.5, this amounts to proving that $\gamma_{r}^{\infty} \circ\left(i_{r}^{\infty}\right)^{*} \circ U_{p}^{n}=U_{p}^{n}$ for any $n>r$, which is clear thanks to Corollary 2.3.3, the description (2.3.6), and the definition (2.3.8a). The proof that (2.3.12b) are inverse isimorphisms is similar, and is left to the reader.

As discussed after Definition B.26, $i_{r}^{\infty}$ is compatible with the $\mathfrak{H}_{r}$-correspondences, and it follows that the isomorphism (2.3.1) resulting from Lemma 2.3.6 is $\mathfrak{H}_{r}$-equivariant (with $U_{p}$ acting on the target via $\left.F_{*}\right)$. Similarly, since the "geometric inertia" action (B.3) of $\Gamma$ on $X_{r}$ is compatible via $i_{r}^{\infty}$ with the trivial action on $I_{r}^{\infty}$ by Proposition B.18, the isomorphism (2.3.1) is equivariant for these actions of $\Gamma$. A nearly identical analysis shows that $\left(i_{r}^{0}\right)^{*}$ is equivariant for the actions of $T_{\ell}^{*}, U_{\ell}^{*}$ (with $U_{p}^{*}$ acting on the target as $\left.\langle p\rangle_{N}^{-1} F_{*}\right)$ and $\langle v\rangle_{N}^{*}$, and that it intertwines $\langle u\rangle^{*}$ on source with $\langle u\rangle$ on target ( $c f$. the preliminary discussion above Proposition 2.3.1). Likewise, $\left(i_{r}^{0}\right)^{*}$ is $\Gamma$-equivariant for the action of $\Gamma$ on $I_{r}^{0}$ via $\langle\chi(\cdot)\rangle^{-1}$. The commutativity of the four diagrams in (2.3.2) is an immediate consequence of the descriptions of the degeneracy mappings $\bar{\rho}, \bar{\sigma}$ on $\bar{X}_{r}^{\mathrm{n}}$ furnished by Proposition B.17 and the explication (2.3.6) of pullback by $i_{r}^{\star}$ in terms of projection.

Finally, that the assertions of Proposition 2.3.1 all hold if $\bar{X}_{*}$ and $\Omega_{I_{*}^{\star}}^{1}(\underline{\mathrm{ss}})$ are replaced by $\bar{X}_{*}^{\mathrm{n}}$ and $\Omega_{I_{*}^{\star}}^{1}$, respectively, follows from a similar - but much simpler - argument. The point is that the maps $\gamma_{r}^{\star}$ of $(2.3 .8 \mathrm{a})-(2.3 .8 \mathrm{~b})$ visibly carry $H^{0}\left(I_{r}^{\star}, \Omega_{I_{r}^{\star} / k}^{1}\right)^{V_{\text {ord }}}$ into $H^{0}\left(\overline{\mathcal{X}}_{r}^{\mathrm{n}}, \Omega_{\bar{X}_{r}^{\mathrm{n}} / k}^{1}\right)$, from which it follows via our argument that they induce the claimed isomorphisms. This completes the proof of Proposition 2.3.1.

Since $\bar{X}_{r}$ is a proper and geometrically connected curve over $\mathbf{F}_{p}$, Proposition A.11 (2) provides short exact sequences of $\mathbf{F}_{p}\left[\Delta / \Delta_{r}\right]$-modules with linear $\Gamma$ and $\mathfrak{H}_{r}^{*}$ (respectively $\mathfrak{H}_{r}$ )-actions

$$
0 \longrightarrow e_{r}^{*} H^{0}\left(\bar{X}_{r}, \omega_{\bar{X}_{r} / \mathbf{F}_{p}}\right) \longrightarrow e_{r}^{*} H^{1}\left(\bar{X}_{r} / \mathbf{F}_{p}\right) \longrightarrow e_{r}^{*} H^{1}\left(\bar{X}_{r}, \mathscr{O}_{\bar{x}_{r}}\right) \longrightarrow 0
$$

respectively

$$
0 \longrightarrow e_{r} H^{0}\left(\bar{X}_{r}, \omega_{\bar{x}_{r} / \mathbf{F}_{p}}\right) \longrightarrow e_{r} H^{1}\left(\bar{X}_{r} / \mathbf{F}_{p}\right) \longrightarrow e_{r} H^{1}\left(\bar{X}_{r}, \mathscr{O}_{\bar{x}_{r}}\right) \longrightarrow 0
$$


which are canonically $\mathbf{F}_{p}$-linearly dual to each other. We likewise have such exact sequences in the case of $\bar{X}_{r}^{\mathrm{n}}$; note that since $\bar{X}_{r}^{\mathrm{n}}$ is smooth, the short exact sequence $H\left(\bar{X}_{r}^{\mathrm{n}} / \mathbf{F}_{p}\right)$ is simply the Hodge filtration of $H_{\mathrm{dR}}^{1}\left(\bar{X}_{r}^{\mathrm{n}} / \mathbf{F}_{p}\right)$.

In what follows, we give $H^{1}\left(I_{r}^{\star}, \mathscr{O}_{I_{r}^{\star}}(-\underline{\mathrm{sS}})\right)$ the structure of $\mathbf{F}_{p}\left[\Delta / \Delta_{r}\right]$-module by decreeing that $u \in \Delta$ act through the adjoint diamond operator $\langle u\rangle^{*}=\left\langle u^{-1}\right\rangle$. The canonical Grothendieck-Serre duality between $H^{1}\left(I_{r}^{\star}, \mathscr{O}_{I_{r}^{\star}}(-\underline{\mathrm{ss}})\right)$ and $H^{0}\left(I_{r}^{\star}, \Omega_{I_{r}^{\star} / \mathbf{F}_{p}}^{1}(\underline{\mathrm{s}})\right)$ then identifies the former with the contragredient of the latter; $c f$. the discussion preceeding Proposition 2.3.1.

Corollary 2.3.7. The absolute Frobenius morphism of $\bar{X}_{r}$ over $\mathbf{F}_{p}$ induces a natural $\mathbf{F}_{p}\left[\Delta / \Delta_{r}\right]$-linear, $\Gamma$-compatible, and $\mathfrak{H}_{r}^{*}$ (respectively $\mathfrak{H}_{r}$ ) equivariant splitting of $(2.3 .13 a)$ (respectively (2.3.13b)). For each $r$ there are natural isomorphisms of split short exact sequences of $\mathbf{F}_{p}\left[\Delta / \Delta_{r}\right]$-modules $(2.3 .14 \mathrm{a})$
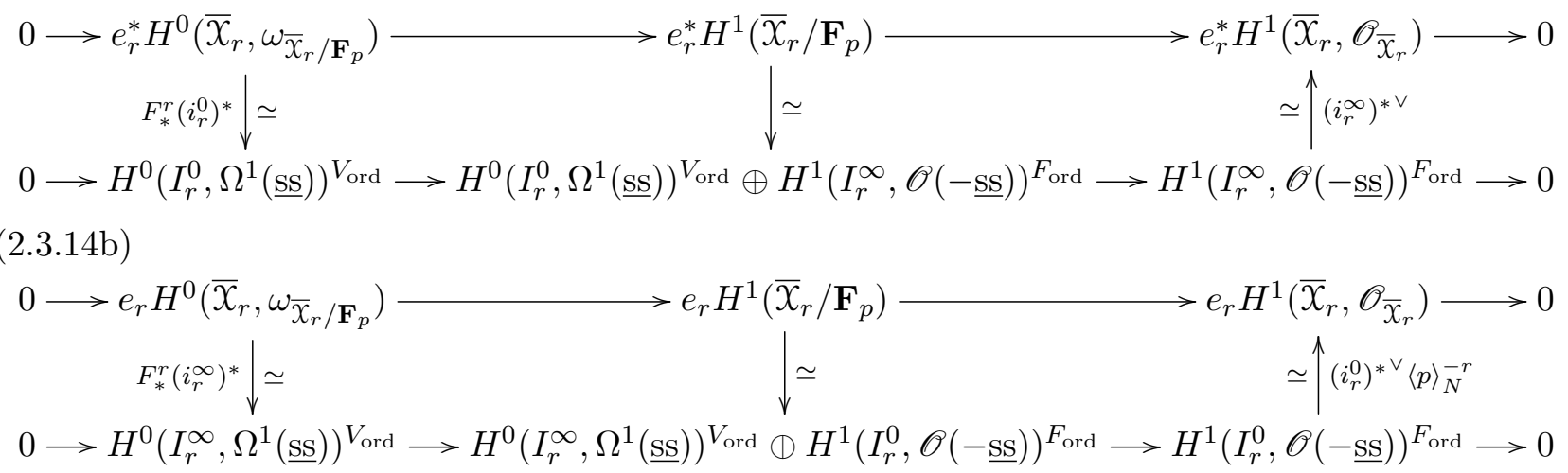

that are compatible with the actions of $\Gamma$ and of the Hecke operators $T_{\ell}^{*}, U_{\ell}^{*}$ and $\langle v\rangle_{N}^{*}$ (respectively $T_{\ell}^{*}, U_{\ell}^{*}$ and $\langle v\rangle_{N}^{*}$ ). The identification $(2.3 .14 a)$ (respectively $(2.3 .14 b)$ ) is moreover compatible with change in $r$ using the trace mappings attached to $\rho: I_{r}^{\star} \rightarrow I_{r-1}^{\star}$ and to $\bar{\rho}: \bar{X}_{r} \rightarrow \bar{X}_{r-1}$ (respectively $\left.\bar{\sigma}: \bar{X}_{r} \rightarrow \bar{X}_{r-1}\right)$. The same statements hold true if we replace $\bar{X}_{r}, \Omega_{I_{r}^{\star}}^{1}(\underline{\mathrm{ss}})$, and $\mathscr{O}_{I_{r}^{\star}}(-\underline{\mathrm{ss}})$ with $\bar{X}_{r}^{\mathrm{n}}, \Omega_{I_{r}^{\star}}^{1}$, and $\mathscr{O}_{I_{r}^{\star}}$, respectively.

Proof. Pullback by the absolute Frobenius endomorphism of $\bar{X}_{r}$ induces an endomorphism of (2.3.13a) which kills $H^{0}\left(\bar{X}_{r}, \omega_{\bar{x}_{r} / \mathbf{F}_{p}}\right)$ and so yields a morphism of $\mathbf{F}_{p}\left[\Delta / \Delta_{r}\right]$-modules

$$
e_{r}^{*} H^{1}\left(\bar{X}_{r}, \mathscr{O}_{\bar{X}_{r}}\right) \longrightarrow e_{r}^{*} H^{1}\left(\bar{X}_{r} / \mathbf{F}_{p}\right)
$$

that is $\Gamma$ and $\mathfrak{H}_{r}^{*}$-compatible and projects to the endomorphism $F^{*}$ of $e_{r}^{*} H^{1}\left(\bar{X}_{r}, \mathscr{O}_{\bar{X}_{r}}\right)$. On the other hand, Proposition 2.3.1 and Grothendieck-Serre duality (see Remark 2.1.5) give a natural $\Gamma$ and $\mathfrak{H}_{r}^{*}$-equivariant isomorphism of $\mathbf{F}_{p}\left[\Delta / \Delta_{r}\right]$-modules

$$
H^{1}\left(I_{r}^{\infty}, \mathscr{O}_{I_{r}^{\infty}}(-\underline{\mathrm{sS}})\right)^{F_{\text {ord }}} \stackrel{\left(i_{r}^{\infty}\right)^{* \vee}}{\longrightarrow} e_{r}^{*} H^{1}\left(\bar{X}_{r}, \mathscr{O}_{\bar{X}_{r}}\right) .
$$

As this isomorphism intertwines $F^{*}$ on source and target, we deduce that $F^{*}$ acts invertibly on $e_{r}^{*} H^{1}\left(\bar{X}_{r}, \mathscr{O}_{\bar{X}_{r}}\right)$. We may therefore pre-compose (2.3.15) with $\left(F^{*}\right)^{-1}$ to obtain a canonical splitting of (2.3.13a), which by construction is $\mathbf{F}_{p}\left[\Delta / \Delta_{r}\right]$-linear and compatible with $\Gamma$ and $\mathfrak{H}_{r}^{*}$. The existence of (2.3.14a) as well as its asserted compatibility with $\Gamma$ and adjoint Hecke operators and with change in $r$ now follows immediately from Proposition 2.3.1 and duality ( $c f$. again Remark 2.1.5). The corresponding assertions for the exact sequence (2.3.13b) and the diagram (2.3.14b) are proved similarly, 
and we leave the details to the reader. A nearly identical argument shows that the same assertions hold true when $\bar{X}_{r}, \Omega_{I_{r}^{\star}}^{1}(\underline{\mathrm{ss}})$, and $\mathscr{O}_{I_{r}^{\star}}(-\underline{\mathrm{ss}})$ are replaced by $\bar{X}_{r}^{\mathrm{n}}, \Omega_{I_{r}^{\star}}^{1}$, and $\mathscr{O}_{I_{r}^{\star}}$, respectively.

Corollary 2.3.8. The exact sequences (2.3.13a) and (2.3.13b) are split short exact sequences of free $\mathbf{F}_{p}\left[\Delta_{1} / \Delta_{r}\right]$-modules whose terms have $\mathbf{F}_{p}\left[\Delta_{1} / \Delta_{r}\right]$-ranks $d, 2 d$, and d, respectively, for $d$ as in Remark 2.2.4. For $s \leq r$, the degeneracy maps $\rho, \sigma: X_{r} \rightrightarrows X_{s}$ induce natural isomorphisms of exact sequences

$$
\begin{gathered}
\rho_{*}: e_{r}^{*} H\left(\bar{X}_{r} / \mathbf{F}_{p}\right)_{\mathbf{F}_{p}\left[\Delta_{1} / \Delta_{s}\right]}^{\otimes} \mathbf{F}_{p}\left[\Delta_{1} / \Delta_{r}\right] \stackrel{\simeq}{\longrightarrow} e_{s}^{*} H\left(\bar{X}_{s} / \mathbf{F}_{p}\right) \\
\sigma_{*}: e_{r} H\left(\bar{X}_{r} / \mathbf{F}_{p}\right)_{\mathbf{F}_{p}\left[\Delta_{1} / \Delta_{s}\right]}^{\otimes} \mathbf{F}_{p}\left[\Delta_{1} / \Delta_{r}\right] \stackrel{\simeq}{\longrightarrow} e_{s} H\left(\bar{X}_{s} / \mathbf{F}_{p}\right)
\end{gathered}
$$

that are $\Gamma$ and $\mathfrak{H}_{r}^{*}\left(\right.$ respectively $\left.\mathfrak{H}_{r}\right)$ equivariant.

Proof. This follows immediately from Proposition 2.2.1 and Corollary 2.3.7.

To formulate an analogue of Corollary 2.3.8 in the case of $\bar{X}_{r}^{\mathrm{n}}$, we proceed as follows. Denote by $\tau: \mathbf{F}_{p}^{\times} \rightarrow \mathbf{Z}_{p}^{\times}$the Teichmüller character, and for any $\mathbf{Z}_{p}\left[\mathbf{F}_{p}^{\times}\right]$-module $M$ and any $j \in \mathbf{Z} /(p-1) \mathbf{Z}$, let

$$
M^{(j)}:=\left\{m \in M: d \cdot m=\tau(d)^{j} m \text { for all } d \in \mathbf{F}_{p}^{\times}\right\}
$$

be the subspace of $M$ on which $\mathbf{F}_{p}^{\times}$acts via $\tau^{j}$. As $\# \mathbf{F}_{p}^{\times}=p-1$ is a unit in $\mathbf{Z}_{p}^{\times}$, the submodule $M^{(j)}$ is a direct summand of $M$. Explicitly, the identity of $\mathbf{Z}_{p}\left[\mathbf{F}_{p}^{\times}\right]$admits the decomposition

$$
1=\sum_{j \in \mathbf{Z} /(p-1) \mathbf{Z}} f_{j} \quad \text { with } \quad f_{j}:=\frac{1}{p-1} \sum_{g \in \mathbf{F}_{p}^{\times}} \tau^{-j}(g) \cdot g
$$

into mutually orthogonal idempotents $f_{j}$, and we have $M^{(j)}=f_{j} M$. In applications, we will consistently need to remove the trivial eigenspace $M^{(0)}$ from $M$, as this eigenspace in the $p$-adic Galois representations we consider is not potentially crystalline at $p$. We will write

$$
f^{\prime}:=\sum_{\substack{j \in \mathbf{Z} /(p-1) \mathbf{Z} \\ j \neq 0}} f_{j}
$$

for the idempotent of $\mathbf{Z}_{p}\left[\mathbf{F}_{p}^{\times}\right]$corresponding to projection away from the 0-eigenspace for $\mathbf{F}_{p}^{\times}$.

Applying these considerations to the identifications of split exact sequences in Corollary 2.3.7, which are compatible with the given diamond operator action of $\Delta=\mathbf{Z}_{p}^{\times} \simeq \mathbf{F}_{p}^{\times} \times \Delta_{1}$ on both rows, we obtain a corresponding identification of split exact sequences of $\tau^{j}$-eigenspaces, for each $j \bmod p-1$. The following is a generalization of [Gross, Proposition 8.10 (2)]:

Lemma 2.3.9. Let $j$ be an integer with $j \not \equiv 0 \bmod p-1$. For each $r$, there are canonical isomorphisms

$$
H^{0}\left(I_{r}^{\star}, \Omega^{1}\right)^{(j)} \stackrel{\simeq}{\longrightarrow} H^{0}\left(I_{r}^{\star}, \Omega^{1}(\underline{\mathrm{ss}})\right)^{(j)} \quad \text { and } \quad H^{1}\left(I_{r}^{\star}, \mathscr{O}(-\underline{\mathrm{ss}})\right)^{(j)} \stackrel{\simeq}{\longrightarrow} H^{1}\left(I_{r}^{\star}, \mathscr{O}\right)^{(j)}
$$

The normalization map $\nu: \bar{X}_{r}^{\mathrm{n}} \rightarrow \bar{X}_{r}$ induces a natural isomorphism of split exact sequences

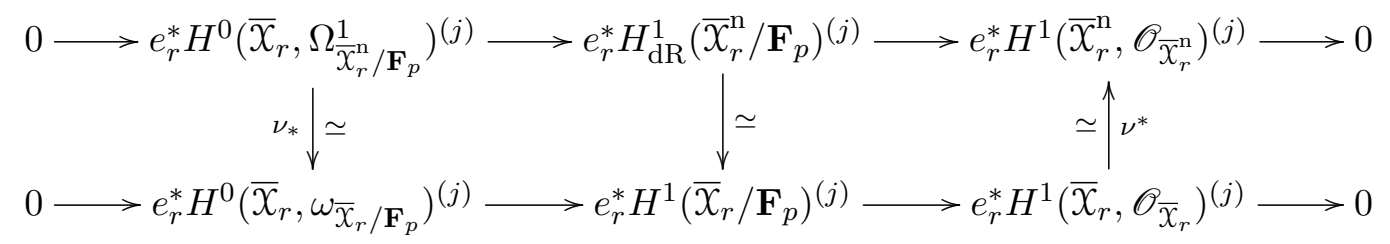


where the central vertical arrow is deduced from the outer two vertical arrows via the splitting of both rows by the Frobenius endomorphism. The same assertions hold if we replace $e_{r}^{*}$ with $e_{r}$.

Proof. The first map in (2.3.19) is injective, as it is simply the canonical inclusion. To see that it is an isomorphism, we may work over $k:=\overline{\mathbf{F}}_{p}$. If $\eta$ is any meromorphic differential on $I_{r}^{\star}$ on which $\mathbf{F}_{p}^{\times}$acts via the character $\tau^{j}$, then since the diamond operators fix every supersingular point on $I_{r}^{\star}$ we have

$$
\operatorname{res}_{x}(\eta)=\operatorname{res}_{x}(\langle u\rangle \eta)=\tau^{j}(u) \operatorname{res}_{x}(\eta)
$$

for any $x \in \underline{\mathrm{ss}}(k)$ and all $u \in \mathbf{F}_{p}^{\times}$thanks to Remark 2.1.2. As $j \not \equiv 0 \bmod p-1$, so $\tau^{j}$ is nontrivial, we must therefore have $\operatorname{res}_{x}(\eta)=0$ for all supersingular points $x$. If in addition $\eta$ is holomorphic outside $\underline{\text { s }}$ with at worst simple poles along $\underline{\mathrm{ss}}$, then $\eta$ must be holomorphic everywhere, so the first map in (2.3.19) is surjective, as desired. As duality interchanges $\langle u\rangle$ with $\langle u\rangle^{*}$, the second mapping in (2.3.19) is dual to the first via our conventions on the $\mathbf{F}_{p}\left[\Delta / \Delta_{r}\right]$-structures of $H^{0}\left(I_{r}^{\star}, \Omega_{I_{r}^{\star} / k}^{1}(\underline{\mathrm{ss}})\right)$ and $H^{1}\left(I_{r}^{\star}, \mathscr{O}_{I_{r}^{\star}}(-\underline{\mathrm{ss}})\right)$, and is therefore an isomorphism as well.

Now for each $j \not \equiv 0 \bmod p-1$, we have a commutative diagram

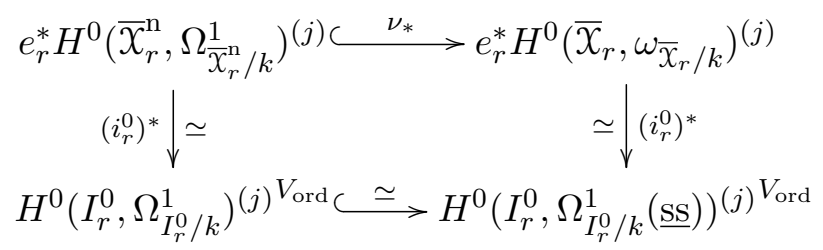

of $\mathbf{F}_{p}\left[\Delta_{1} / \Delta_{r}\right]$-modules in which the two vertical arrows are isomorphisms by Proposition 2.3.1 and the bottom horizontal mapping is an isomorphism as we have just seen. We conclude that the top horizontal arrow of (2.3.21) is an isomorphism as well. Thus, the left vertical map in (2.3.20) is an isomorphism. A similar argument shows that the analogue of (2.3.21) with $e_{r}$ in place of $e_{r}^{*}$ and $I_{r}^{\infty}, i_{r}^{\infty}$ in place of $I_{r}^{0}$ and $i_{r}^{0}$, respectively, is likewise a commutative diagram of isomorphisms. Dualizing this analogue then shows that the right vertical map of (2.3.20) is an isomorphism as well. The diagram (2.3.20) then follows at once from the fact the both rows are canonically split by the Frobenius endomorphism, thanks to Corollary 2.3.7. A nearly identical argument shows that the same assertions hold if we replace $e_{r}^{*}$ with $e_{r}$ throughout.

If $A$ is any $\mathbf{Z}_{p}\left[\mathbf{F}_{p}^{\times}\right]$-algebra and $a \in A$, we will write $a^{\prime}:=f^{\prime} a$ for the product of $a$ with the idempotent $f^{\prime}$ of (2.3.18). We will apply this to $A=\mathfrak{H}_{r}, \mathfrak{H}_{r}^{*}$ viewed as $\mathbf{Z}_{p}\left[\mathbf{F}_{p}^{\times}\right]$-algebras via the diamond and adjoint diamond operators, with $a=e_{r}$ and $e_{r}^{*}$, respectively.

Proposition 2.3.10. For each $r$ there are isomorphisms of split short exact sequences of $\mathbf{F}_{p}\left[\Delta_{1} / \Delta_{r}\right]$ modules

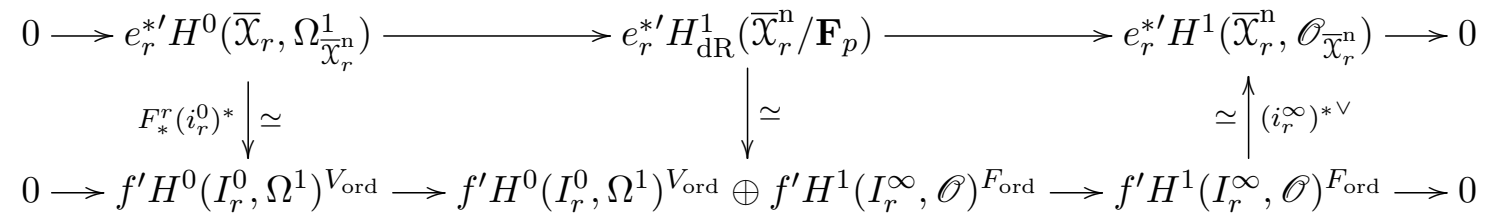




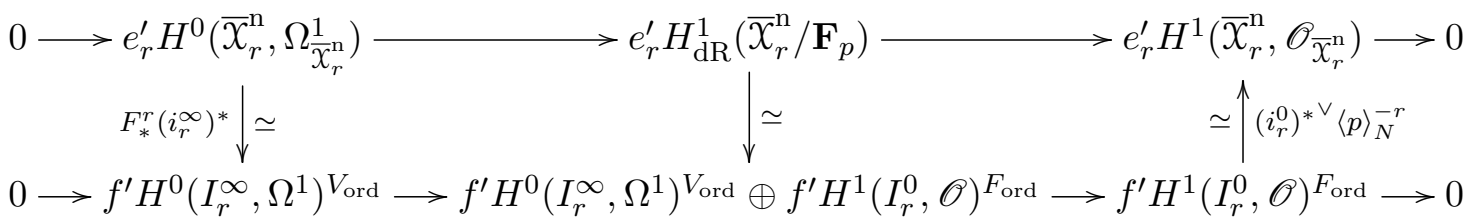

Setting $d^{\prime}:=\sum_{k=3}^{p} d_{k}$ where $d_{k}:=\operatorname{dim}_{\mathbf{F}_{p}} S_{k}\left(\Gamma_{1}(N) ; \mathbf{F}_{p}\right)^{\text {ord }}$ as in Remark 2.2.4, the terms in the top rows of (2.3.22a) and (2.3.22b) are free over $\mathbf{F}_{p}\left[\Delta_{1} / \Delta_{r}\right]$ of ranks $d^{\prime}, 2 d^{\prime}$, and $d^{\prime}$. The identification $(2.3 .22 a)$ (respectively $(2.3 .22 b))$ is equivariant for the actions of $\Gamma$ and the Hecke operators $T_{\ell}^{*}, U_{\ell}^{*}$, and $\langle v\rangle_{N}^{*}$ (respectively $T_{\ell}, U_{\ell}$, and $\langle v\rangle_{N}$ ), and compatible with change in $r$ using the trace mappings attached to $\rho: I_{r}^{\star} \rightarrow I_{s}^{\star}$ and to $\bar{\rho}: \bar{X}_{r} \rightarrow \bar{X}_{s}$ (respectively $\bar{\sigma}: \bar{X}_{r} \rightarrow \bar{X}_{s}$ ).

Proof. This follows immediately from Corollaries 2.3.7-2.3.8 and Lemma 2.3.9, using the fact that the group ring $\mathbf{F}_{p}\left[\Delta_{1} / \Delta_{r}\right]$ is local, so any projective $\mathbf{F}_{p}\left[\Delta_{1} / \Delta_{r}\right]$-module is free.

\section{ORdinary $\Lambda$-ADiC DE Rham COHOMOlogy}

In this section, we will state and prove our main results as described in $\S 1.2$. Throughout, we will keep the notation of $\S 1.2$ and the introduction of $\S 2$. Recall, in particular, that for a ring $A$ we often write $\Lambda_{A, r}:=A\left[\Delta_{1} / \Delta_{r}\right]$ and define $\Lambda_{A}:=\varliminf_{r} \Lambda_{A, r}$.

3.1. The formalism of towers. In this preliminary section, we set up a general commutative algebra framework for dealing with the various projective limits of cohomology modules that we will encounter.

Definition 3.1.1. A tower of rings is an inductive system $\mathscr{A}:=\left\{A_{r}\right\}_{r \geq 1}$ of local rings with local transition maps. A morphism of towers $\mathscr{A} \rightarrow \mathscr{A}^{\prime}$ is a collection of local ring homomorphisms $A_{r} \rightarrow A_{r}^{\prime}$ which are compatible with change in $r$. A tower of $\mathscr{A}$-modules $\mathscr{M}$ consists of the following data:

(1) For each integer $r \geq 1$, an $A_{r}$-module $M_{r}$.

(2) A collection of $A_{r}$-module homomorphisms $\rho_{r, s}: M_{r} \rightarrow M_{s} \otimes_{A_{s}} A_{r}$ for each pair of integers $r \geq s \geq 1$, which are compatible in the obvious way under composition.

A morphism of towers of $\mathscr{A}$-modules $\mathscr{M} \rightarrow \mathscr{M}^{\prime}$ is a collection of $A_{r}$-module homomorphisms $M_{r} \rightarrow M_{r}^{\prime}$ which are compatible with change in $r$ in the evident manner. For a tower of rings $\mathscr{A}=\left\{A_{r}\right\}$, we will write $A_{\infty}$ for the inductive limit, and for a tower of $\mathscr{A}$-modules $\mathscr{M}=\left\{M_{r}\right\}$ and any $A_{\infty}$-algebra $B$ we set

$$
M_{B}:=\underbrace{\lim }_{r}\left(M_{r} \otimes_{A_{r}} B\right) \quad \text { and write simply } \quad M_{\infty}:=M_{A_{\infty}},
$$

with the projective limit taken with respect to the induced transition maps.

Lemma 3.1.2. Let $\mathscr{A}=\left\{A_{r}\right\}_{r \geq 0}$ be a tower of rings and suppose that $I_{r} \subseteq A_{r}$ is a sequence of proper ideals such that the image of $I_{r}$ in $A_{r+1}$ is contained in $I_{r+1}$ for all $r$. Set $\bar{A}_{r}:=A_{r} / I_{r}$ for all $r$. Let $\mathscr{M}=\left\{M_{r}, \rho_{r, s}\right\}$ be a tower of $\mathscr{A}$-modules equipped with an action ${ }^{6}$ of $\Delta_{1}$ by $\mathscr{A}$-automorphisms. Suppose that $M_{r}$ is free of finite rank over $A_{r}$ for all $r$, and that $\Delta_{r}$ acts trivially on $M_{r}$. Let $B$ be an $A_{\infty}$-algebra, and observe that $M_{B}$ is canonically a module over the completed group ring $\Lambda_{B}$. Assume that that the following conditions hold for $r>0$ :

(3.1.1a) $\bar{M}_{r}:=M_{r} / I_{r} M_{r}$ is a free $\bar{A}_{r}\left[\Delta_{1} / \Delta_{r}\right]$-module of rank $d$ that is independent of $r$.

(3.1.1b) For all $s \leq r$ the induced maps $\bar{\rho}_{r, s}: \bar{M}_{r} \longrightarrow \bar{M}_{s} \otimes_{\bar{A}_{s}} \bar{A}_{r}$ are surjective.

\footnotetext{
${ }^{6}$ That is, a homomorphism of groups $\Delta_{1} \rightarrow \operatorname{Aut}_{\mathscr{A}}(\mathscr{M})$, or equivalently, an $A_{r}$-linear action of $\Delta_{1}$ on $M_{r}$ for each $r$ that is compatible with change in $r$.
} 
Then:

(1) $M_{r}$ is a free $A_{r}\left[\Delta_{1} / \Delta_{r}\right]$-module of rank $d$ for all $r$.

(2) The induced maps of $A_{r}\left[\Delta_{1} / \Delta_{s}\right]$-modules

$$
M_{r} \otimes_{A_{r}\left[\Delta_{1} / \Delta_{r}\right]} A_{r}\left[\Delta_{1} / \Delta_{s}\right] \longrightarrow M_{s} \otimes_{A_{s}} A_{r}
$$

are isomorphisms for all $r \geq s$.

(3) $M_{B}$ is a finite free $\Lambda_{B}$-module of rank $d$.

(4) For each $r$, the canonical map

$$
M_{B} \otimes_{\Lambda_{B}} \Lambda_{B, r} \longrightarrow M_{r} \otimes_{A_{r}} B
$$

is an isomorphism of $\Lambda_{B, r}$-modules.

(5) If $B^{\prime}$ is any $B$-algebra, then the canonical map

$$
M_{B} \otimes_{\Lambda_{B}} \Lambda_{B^{\prime}} \longrightarrow M_{B^{\prime}}
$$

is an isomorphism of finite free $\Lambda_{B^{\prime}}$-modules.

Proof. Let us fix $r$ and choose a generator $\gamma$ of $\Delta_{1} / \Delta_{r}$. The module $M_{r}$ is obviously finite over $\Lambda_{A_{r}, r}$ (as it is even finite over $A_{r}$ ), so by hypothesis (3.1.1a) we may choose $m_{1}, \ldots, m_{d} \in M_{r}$ with the property that the images $\bar{m}_{i}$ of the $m_{i}$ in $\bar{M}_{r}=M_{r} / I_{r} M_{r}$ freely generate $\bar{M}_{r}$ as an $\bar{A}_{r}\left[\Delta_{1} / \Delta_{r}\right]-$ module. Then the images of the $p^{r-1} d$ elements $\left\{\gamma^{i} m_{j}\right\}_{i, j}$ for $i=1, \ldots, p^{r-1}$ and $j=1, \ldots, d$ freely generate $\bar{M}_{r}$ as an $\bar{A}_{r}$-module, and the (by assumption) free $A_{r}$-module $M_{r}$ must have rank $p^{r-1} d$. By Nakayama's Lemma, the set $\left\{\gamma^{i} m_{j}\right\}_{i, j}$ is then a minimal set of generators of the free $A_{r}$-module $M_{r}$, with cardinality equal to the $A_{r}$-rank of $M_{r}$. As such, it must freely generate $M_{r}$ over $A_{r}$. It follows that $m_{1}, \ldots, m_{d}$ freely generate $M_{r}$ over $\Lambda_{A_{r}, r}$, giving (1).

To prove (2), note that our assumption (3.1.1b) that the maps $\bar{\rho}_{r, s}$ are surjective for all $r \geq s$ implies that the same is true of the maps $\rho_{r, s}$ (by Nakayama's Lemma) and hence that the induced map of $\Lambda_{A_{r}, s^{-}}$-modules in (2) is surjective. As this map is then a surjective map of free $\Lambda_{A_{r}, s^{-m o d u l e s}}$ of the same rank $d$, it must be an isomorphism.

Since the kernel of the canonical surjection $\Lambda_{A_{r}, r} \rightarrow \Lambda_{A_{r}, s}$ lies in the radical of $\Lambda_{A_{r}, r}$, we deduce by Nakayama's Lemma that any lift to $M_{r}$ of a $\Lambda_{A_{r}, s}$-basis of $M_{s} \otimes_{A_{s}} A_{r}$ is a $\Lambda_{A_{r}, r}$-basis of $M_{r}$. It follows that for all $r \geq 0$, we may choose a $\Lambda_{B, r}$-basis $e_{1 r}, \ldots, e_{d r}$ of the free $\Lambda_{B, r}$-module $M_{r} \otimes_{A_{r}} B$ with the property that $e_{i r}$ is carried to $e_{i s}$ under the induced map $M_{r} \otimes_{A_{r}} B \rightarrow M_{s} \otimes_{A_{s}} B$ for all $s \leq r$. These choices yield isomorphisms $\Lambda_{B, r}^{\oplus d} \simeq M_{r} \otimes_{A_{r}} B$ which are compatible with change in $r$ using the canonical projections on each factor on the left side and the induced transition maps on the right, and it follows that $M_{B}$ is a free $\Lambda_{B}$-module of rank $d$ for any $A_{\infty}$-algebra $B$.

We have seen that the mappings $\rho_{r, s}$ are surjective for all $r \geq s$, and it follows that the canonical map $M_{B} \rightarrow M_{r} \otimes_{A_{r}} B$ is surjective as well. Since the mapping of (4) is obtained from this surjection by extension of scalars (keeping in mind the natural identification $\left.\left(M_{r} \otimes_{A_{r}} B\right) \otimes_{\Lambda_{B}} \Lambda_{B, r} \simeq M_{r} \otimes_{A_{r}} B\right)$, we deduce that (4) is likewise surjective. From (1) and (3) we conclude that the mapping in (4) is a surjection of free $\Lambda_{B, r}$-modules of the same rank and is hence an isomorphism as claimed.

It remains to prove (5). Extending scalars, the canonical maps $M_{B} \rightarrow M_{r} \otimes_{A_{r}} B$ induce surjections

$$
M_{B} \otimes_{\Lambda_{B}} \Lambda_{B^{\prime}} \longrightarrow\left(M_{r} \otimes_{A_{r}} B\right) \otimes_{\Lambda_{B}} \Lambda_{B^{\prime}} \simeq M_{r} \otimes_{A_{r}} B^{\prime}
$$

that are compatible in the evident manner with change in $r$. Passing to inverse limits gives the mapping $M_{B} \otimes_{\Lambda_{B}} \Lambda_{B^{\prime}} \rightarrow M_{B^{\prime}}$ of (5). Due to (3), this is then a map of finite free $\Lambda_{B^{\prime}}$-modules of 
the same rank, so to check that it is an isomorphism it suffices by Nakayama's Lemma to do so after applying $\otimes_{\Lambda_{B^{\prime}}} \Lambda_{B^{\prime}, r}$, which is an immediate consequence of (4).

We record the following elementary commutative algebra fact, which will be of use to us in the sequel to this paper [Cais3]:

Lemma 3.1.3. Let $A \rightarrow B$ be a local homomorphism of local rings which makes $B$ into a flat $A$ algebra, and let $M$ be an arbitrary $A$-module. Then $M$ is a free $A$-module of finite rank if and only if $M \otimes_{A} B$ is a free $B$-module of finite rank.

Proof. First observe that since $A \rightarrow B$ is local and flat, it is faithfully flat. We write $M=\lim M_{\alpha}$ as the direct limit of its finitely generated $A$-submodules, whence $M \otimes_{A} B=\underline{\lim }\left(M_{\alpha} \otimes_{A} B\right)$ with each of $M_{\alpha} \otimes_{A} B$ naturally a finitely generated $B$-submodule of $M \otimes_{A} B$. Assume that $M \otimes_{A} B$ is finitely generated as a $B$-module. Then there exists $\alpha$ with $M_{\alpha} \otimes_{A} B \rightarrow M \otimes_{A} B$ surjective, and as $B$ is faithfully flat over $A$, this implies that $M_{\alpha} \rightarrow M$ is surjective, whence $M$ is finitely generated over $A$. Suppose in addition that $M \otimes_{A} B$ is free as a $B$-module. In particular, $M \otimes_{A} B$ is $B$-flat, which implies by faithful flatness of $B$ over $A$ that $M$ is $A$-flat (see, e.g. [Matsumura, Exercise 7.1]). Then $M$ is a finite flat module over the local ring $A$, whence it is free as an $A$-module by [Matsumura, Theorem 7.10].

Finally, we analyze duality for towers with $\Delta_{1}$-action.

Lemma 3.1.4. With the notation of Lemma 3.1.2, let $\mathscr{M}:=\left\{M_{r}, \rho_{r, s}\right\}$ and $\mathscr{M}^{\prime}:=\left\{M_{r}^{\prime}, \rho_{r, s}^{\prime}\right\}$ be two towers of $\mathscr{A}$-modules with $\Delta_{1}$-action satisfying (3.1.1a) and (3.1.1b). Suppose that for each $r$ there exist $A_{r}$-linear perfect duality pairings

$$
\langle\cdot, \cdot\rangle_{r}: M_{r} \times M_{r}^{\prime} \longrightarrow A_{r}
$$

with respect to which $\delta$ is self-adjoint for all $\delta \in \Delta_{1}$, and which satisfy the compatibility condition ${ }^{7}$

$$
\left\langle\rho_{r, s} m, \rho_{r, s}^{\prime} m^{\prime}\right\rangle_{s}=\sum_{\delta \in \Delta_{s} / \Delta_{r}}\left\langle m, \delta^{-1} m^{\prime}\right\rangle_{r}
$$

for all $r \geq s$. Then for each $r$, the pairings $(\cdot, \cdot)_{r}: M_{r} \times M_{r}^{\prime} \longrightarrow \Lambda_{A_{r}, r}$ defined by

$$
\left(m, m^{\prime}\right)_{r}:=\sum_{\delta \in \Delta_{1} / \Delta_{r}}\left\langle m, \delta^{-1} m^{\prime}\right\rangle_{r} \cdot \delta
$$

are $\Lambda_{A_{r}, r}$-bilinear and perfect, and compile to give a $\Lambda_{B}$-linear perfect pairing

$$
(\cdot, \cdot)_{\Lambda_{B}}: M_{B} \times M_{B}^{\prime} \longrightarrow \Lambda_{B}
$$

In particular, $M_{B}^{\prime}$ and $M_{B}$ are canonically $\Lambda_{B}$-linearly dual to each other.

Proof. An easy reindexing argument shows that $(\cdot, \cdot)_{r}$ is $\Lambda_{A_{r}, r}$-linear in the right factor, from which it follows that it is also $\Lambda_{A_{r}, r}$-linear in the left due to our assumption that $\delta \in \Delta_{1}$ is self-adjoint with respect to $\langle\cdot, \cdot\rangle_{r}$. To prove that $(\cdot, \cdot)_{r}$ is a perfect duality pairing, we analyze the $\Lambda_{A_{r}, r}$-linear map

$$
M_{r} \stackrel{m \mapsto(m, \cdot)_{r}}{\longrightarrow} \operatorname{Hom}_{\Lambda_{A_{r}, r}}\left(M_{r}^{\prime}, \Lambda_{A_{r}, r}\right) .
$$

\footnotetext{
${ }^{7}$ By abuse of notation, for any map of rings $A \rightarrow B$ and any $A$-bilinear pairing of $A$-modules $\langle\cdot, \cdot\rangle: M \times M^{\prime} \rightarrow A$, we again write $\langle\cdot, \cdot\rangle: M_{B} \times M_{B}^{\prime} \rightarrow B$ for the $B$-bilinear pairing induced by extension of scalars.
} 
Due to Lemma 3.1.2, both $M_{r}$ and $M_{r}^{\prime}$ are free $\Lambda_{A_{r}, r}$-modules, necessarily of the same rank by the existence of the perfect $A_{r}$-duality pairing (3.1.2). It follows that (3.1.4) is a homomorphism of free $\Lambda_{A_{r}, r}$-modules of the same rank. To show that it is an isomorphism it therefore suffices to prove it is surjective, which may be checked after extension of scalars along the augmentation map $\Lambda_{A_{r}, r} \rightarrow A_{r}$ by Nakayama's Lemma. Consider the diagram

$$
\begin{aligned}
& M_{r} \underset{\Lambda_{A_{r}, r}}{\otimes} A_{r} \stackrel{(3.1 .4) \otimes 1}{\longrightarrow} \operatorname{Hom}_{\Lambda_{A_{r}, r}}\left(M_{r}^{\prime}, \Lambda_{A_{r}, r}\right) \underset{\Lambda_{A_{r}, r}}{\otimes} A_{r} \stackrel{\xi}{\simeq} \operatorname{Hom}_{A_{r}}\left(M_{r}^{\prime} \underset{\Lambda_{A_{r}, r}}{\otimes} A_{r}, A_{r}\right) \\
& \rho_{r, 1} \otimes 1 \downarrow \simeq \quad \simeq \uparrow\left(\rho_{r, 1}^{\prime} \otimes 1\right)^{\vee} \\
& M_{1} \underset{A_{1}}{\otimes} A_{r} \longrightarrow \operatorname{Hom}_{A_{r}}\left(M_{1}^{\prime} \underset{A_{1}}{\otimes} A_{r}, A_{r}\right)
\end{aligned}
$$

where $\xi$ is the canonical map that sends $f \otimes \alpha$ to $\alpha(f \otimes 1)$ for any $\Lambda_{A_{r}, r}$-linear functional $f: M_{r}^{\prime} \rightarrow \Lambda_{A_{r}, r}$ and any $\alpha \in A_{r}$, and the bottom horizontal arrow is obtained by $A_{r}$-linearly extending the canonical duality map $m \mapsto\langle m, \cdot\rangle_{1}$. On the one hand, the vertical maps in (3.1.5) are isomorphisms thanks to Lemma 3.1.2 (2), while the map $\xi$ and the bottom horizontal arrow are isomorphisms because arbitrary extension of scalars commutes with linear duality of finitely generated free modules. ${ }^{8}$ On the other hand, this diagram commutes because (3.1.3) guarantees the relation

$$
\left\langle\rho_{r, 1} m, \rho_{r, 1}^{\prime} m^{\prime}\right\rangle_{1} \stackrel{(3.1 .3)}{=} \sum_{\delta \in \Delta_{1} / \Delta_{r}}\left\langle m, \delta^{-1} m^{\prime}\right\rangle_{r} \equiv\left(m, m^{\prime}\right)_{r} \bmod I_{\Delta}
$$

where $I_{\Delta}=\operatorname{ker}\left(\Lambda_{A_{r}, r} \rightarrow A_{r}\right)$ is the augmentation ideal. We conclude that (3.1.4) is an isomorphism, as desired. The argument that the corresponding map with the roles of $M_{r}$ and $M_{r}^{\prime}$ interchanged is an isomorphism proceeds mutatis mutandis.

Using the definition of $(\cdot, \cdot)_{r}$ and (3.1.3), one has more generally that

$$
\left(\rho_{r, s} m, \rho_{r, s}^{\prime} m^{\prime}\right)_{s} \equiv\left(m, m^{\prime}\right)_{r} \bmod \operatorname{ker}\left(\Lambda_{A_{r}, r} \rightarrow \Lambda_{A_{r}, s}\right)
$$

for all $r \geq s$. In particular, the pairings $(\cdot, \cdot)_{r}$ induce, by extension of scalars, a $\Lambda_{B}$-bilinear pairing

$$
(\cdot, \cdot)_{\Lambda_{B}}: M_{B} \times M_{B}^{\prime} \longrightarrow \Lambda_{B}
$$

which satisfies the specialization property

$$
(\cdot, \cdot)_{\Lambda_{B}} \equiv(\cdot, \cdot)_{r} \bmod \operatorname{ker}\left(\Lambda_{B} \rightarrow \Lambda_{B, r}\right) .
$$

From $(\cdot, \cdot)_{\Lambda_{B}}$ we obtain in the usual way duality morphisms

$$
M_{B} \stackrel{m \mapsto(m, \cdot)_{\Lambda_{B}}}{\longrightarrow} \operatorname{Hom}_{\Lambda_{B}}\left(M_{B}^{\prime}, \Lambda_{B}\right) \quad \text { and } \quad M_{B}^{\prime} \stackrel{m^{\prime} \mapsto\left(\cdot, m^{\prime}\right) \Lambda_{B}}{\longrightarrow} \operatorname{Hom}_{\Lambda_{B}}\left(M_{B}, \Lambda_{B}\right)
$$

which we wish to show are isomorphisms. Due to Lemma 3.1.2 (3), each of (3.1.7) is a map of finite free $\Lambda_{B}$-modules of the same rank, so we need only show that these mappings are surjective. As the kernel of $\Lambda_{B} \rightarrow \Lambda_{B, r}$ is contained in the radical of $\Lambda_{B}$, we may by Nakayama's Lemma check such surjectivity after extension of scalars along $\Lambda_{B} \rightarrow \Lambda_{B, r}$ for any $r$, where it follows from (3.1.6) and

\footnotetext{
${ }^{8}$ Quite generally, for any ring $R$, any $R$-modules $M, N$, and any $R$-algebra $S$, the canonical map

$$
\xi_{M}: \operatorname{Hom}_{R}(M, N) \otimes_{R} S \longrightarrow \operatorname{Hom}_{S}\left(M \otimes_{R} S, N \otimes_{R} S\right)
$$

sending $f \otimes s$ to $s\left(f \otimes \mathrm{id}_{S}\right)$ is an isomorphism if $M$ is finite and free over $R$. Indeed, the map $\xi_{R}$ is visibly an isomorphism, and one checks that $\xi_{M_{1} \oplus M_{2}}$ is naturally identified with $\xi_{M_{1}} \oplus \xi_{M_{2}}$.
} 
the fact that $M_{r}$ and $M_{r}^{\prime}$ are free $\Lambda_{A_{r}, r}$-modules, so that the extension of scalars of the perfect duality pairing $(\cdot, \cdot)_{r}$ along the canonical map $\Lambda_{A_{r}, r} \rightarrow \Lambda_{B, r}$ is again perfect.

3.2. Ordinary families of de Rham cohomology. Let $\left\{X_{r} / T_{r}\right\}_{r \geq 0}$ be the tower of modular curves introduced in $\S \mathrm{B}$. As $X_{r}$ is regular and proper flat over $T_{r}=\operatorname{Spec}\left(R_{r}\right)$ with geometrically reduced fibers, it is a curve in the sense of Definition A.1 (thanks to Corollary A.3) which moreover satisfies the hypotheses of Proposition A.10. Abbreviating

$$
H^{0}\left(\omega_{r}\right):=H^{0}\left(\mathcal{X}_{r}, \omega_{X_{r} / S_{r}}\right), \quad H_{\mathrm{dR}, r}^{1}:=H^{1}\left(\mathcal{X}_{r} / R_{r}\right), \quad H^{1}\left(\mathscr{O}_{r}\right):=H^{1}\left(\mathcal{X}_{r}, \mathscr{O}_{X_{r}}\right),
$$

Proposition A.10 (2) provides a canonical short exact sequence $H\left(X_{r} / R_{r}\right)$ of finite free $R_{r}$-modules

$$
0 \longrightarrow H^{0}\left(\omega_{r}\right) \longrightarrow H_{\mathrm{dR}, r}^{1} \longrightarrow H^{1}\left(\mathscr{O}_{r}\right) \longrightarrow 0
$$

which recovers the Hodge filtration of $H_{\mathrm{dR}}^{1}\left(X_{r} / K_{r}\right)$ after inverting $p$.

The Hecke correspondences on $\mathcal{X}_{r}$ induce, via Proposition A.10 (4) and the discussion surrounding Definition A.15, canonical actions of $\mathfrak{H}_{r}$ and $\mathfrak{H}_{r}^{*}$ on $H\left(X_{r} / R_{r}\right)$ via $R_{r}$-linear endomorphisms. In particular, $H\left(X_{r} / R_{r}\right)$ is canonically a short exact sequence of $\Lambda$-modules via the diamond and adjoint diamond operator maps $\Lambda \hookrightarrow \mathfrak{H}$ and $\Lambda \hookrightarrow \mathfrak{H}^{*}$ as in $\S 1.4$. Similarly, pullback along (B.3) yields $R_{r^{-}}$ linear morphisms $H\left(\left(X_{r}\right)_{\gamma} / R_{r}\right) \rightarrow H\left(X_{r} / R_{r}\right)$ for each $\gamma \in \Gamma$; using the fact that hypercohomology commutes with flat base change (by Cech theory), we obtain an action of $\Gamma$ on $H\left(X_{r} / R_{r}\right)$ which is $R_{r}$-semilinear over the canonical action of $\Gamma$ on $R_{r}$ and which commutes with the actions of $\mathfrak{H}_{r}$ and $\mathfrak{H}_{r}^{*}$ as the Hecke operators are defined over $K_{0}=\mathbf{Q}_{p}$.

For $r \geq s$, we will need to work with the base change $X_{s} \times_{T_{s}} T_{r}$, which is a curve over $T_{r}$ thanks to Proposition A.2. Although $X_{s} \times_{T_{s}} T_{r}$ need no longer be regular as $T_{r} \rightarrow T_{s}$ is not smooth when $r>s$, we claim that it is necessarily normal. Indeed, this follows from the more general assertion:

Lemma 3.2.1. Let $V$ be a discrete valuation ring and $A$ a finite type Cohen-Macaulay $V$-algebra ${ }^{9}$ with smooth generic fiber and geometrically reduced special fiber. Then $A$ is normal.

Proof. We claim that $A$ satisfies Serre's " $R_{1}+S_{2}$ "-criterion for normality [Matsumura, Theorem 23.8]. As $A$ is by hypothesis a Cohen-Macualay ring, it verifies $S_{i}$ for all $i \geq 0$, and we need only show that each localization of $A$ at a prime ideal of codimension 1 is regular. Since $A$ has geometrically reduced special fiber, this special fiber is in particular smooth at its generic points. As $A$ is flat over $V$ (again by hypothesis), we deduce that the (open) $V$-smooth locus in $\operatorname{Spec} A$ contains the generic points of the special fiber and hence contains all codimension-1 points (as the generic fiber of $\operatorname{Spec} A$ is assumed to be smooth). Thus $A$ is $R_{1}$, as desired.

We conclude that $X_{s} \times_{T_{s}} T_{r}$ is a normal curve, and we obtain from Proposition A.10 a canonical short exact sequence of finite free $R_{r}$-modules $H\left(X_{s} \times_{T_{s}} T_{r} / R_{r}\right)$ which recovers the Hodge filtration of $H_{\mathrm{dR}}^{1}\left(X_{s} / K_{r}\right)$ after inverting $p$. As hypercohomology commutes with flat base change and the formation of the relative dualizing sheaf and the structure sheaf are compatible with arbitrary base change, we have a natural isomorphism of short exact sequences of free $R_{r}$-modules

$$
H\left(X_{s} \times_{T_{s}} T_{r} / R_{r}\right) \simeq H\left(X_{s} / R_{s}\right) \otimes_{R_{s}} R_{r} .
$$

In particular, we have $R_{r}$-linear actions of $\mathfrak{H}_{s}^{*}, \mathfrak{H}_{s}$ and an $R_{r}$-semilinear action of $\Gamma$ on $H\left(X_{s} \times_{T_{s}} T_{r} / R_{r}\right)$. These actions moreover commute with one another.

\footnotetext{
${ }^{9}$ By definition, a finite type algebra $A$ over a noetherian ring $V$ is a Cohen-Macaulay $V$-algebra when it is $V$-flat and the fiber algebras over points of $\operatorname{Spec}(V)$ are Cohen-Macaulay rings. Due to $V$-flatness, when $V$ is itself a Cohen-Macaulay ring, the condition on all fiber algebras is equivalent to $A$ being a Cohen-Macaulay ring as well.
} 
Consider now the canonical degeneracy map $\rho: X_{r} \rightarrow X_{s} \times_{T_{s}} T_{r}$ of curves over $T_{r}$ induced by (B.6). As $X_{r}$ and $X_{s} \times_{T_{s}} T_{r}$ are normal and proper curves over $T_{r}$, we obtain from Proposition A.10 (4) canonical trace mappings of short exact sequences

$$
\rho_{*}: H\left(X_{r} / R_{r}\right) \longrightarrow H\left(X_{s} \times_{T_{s}} T_{r} / R_{r}\right) \simeq H\left(X_{s} / R_{s}\right) \otimes_{R_{s}} R_{r}
$$

which recover the usual trace mappings on de Rham cohomology after inverting $p$; as such, these mappings are Hecke and $\Gamma$-equivariant, and compatible with change in $r, s$ in the obvious way. Tensoring the maps (3.2.4) over $R_{r}$ with $R_{\infty}$, we obtain projective systems of finite free $R_{\infty}$-modules with semilinear $\Gamma$-action and commuting, linear $\mathfrak{H}^{*}:={\underset{\varliminf}{\leftarrow}}_{r} \mathfrak{H}_{r}^{*}$ action.

Definition 3.2.2. We write

$$
H^{0}(\omega):=\underbrace{\lim }_{r}\left(H^{0}\left(\omega_{r}\right) \underset{R_{r}}{\otimes} R_{\infty}\right), \quad H_{\mathrm{dR}}^{1}:=\underbrace{\lim }_{r}\left(H_{\mathrm{dR}, r}^{1} \underset{R_{r}}{\otimes} R_{\infty}\right), \quad H^{1}(\mathscr{O}):=\underbrace{\lim }_{r}\left(H^{1}\left(\mathscr{O}_{r}\right) \underset{R_{r}}{\otimes} R_{\infty}\right)
$$

for the limits with respect to the maps induced by $\rho_{*}$, which are naturally $\Lambda_{R_{\infty}}=R_{\infty} \llbracket \Delta_{1} \rrbracket$-modules via the adjoint diamond operators and are equipped with a semilinear $\Gamma$-action and a linear $\mathfrak{H}^{*}$-action.

Theorem 3.2.3. Let $e^{*}$ be the idempotent of $\mathfrak{H}^{*}$ associated to $U_{p}^{*}$ and let $d$ be the positive integer defined as in Proposition 2.2.1 (1). Then $e^{*} H^{0}(\omega), e^{*} H_{\mathrm{dR}}^{1}$ and $e^{*} H^{1}(\mathscr{O})$ are free $\Lambda_{R_{\infty}}$-modules of ranks $d, 2 d$, and $d$ respectively, and there is a canonical short exact sequence of free $\Lambda_{R_{\infty}}$-modules with linear $\mathfrak{H}^{*}$-action and $R_{\infty}$-semilinear $\Gamma$-action

$$
0 \longrightarrow e^{*} H^{0}(\omega) \longrightarrow e^{*} H_{\mathrm{dR}}^{1} \longrightarrow e^{*} H^{1}(\mathscr{O}) \longrightarrow 0 \text {. }
$$

For each positive integer $r$, applying $\otimes_{\Lambda_{R_{\infty}}} R_{\infty}\left[\Delta_{1} / \Delta_{r}\right]$ to (3.2.5) yields the short exact sequence

$$
0 \longrightarrow e^{*} H^{0}\left(\omega_{r}\right) \underset{R_{r}}{\otimes} R_{\infty} \longrightarrow e^{*} H_{\mathrm{dR}}^{1} \underset{R_{r}}{\otimes} R_{\infty} \longrightarrow e^{*} H^{1}(\mathscr{O}) \underset{R_{r}}{\otimes} R_{\infty} \longrightarrow 0,
$$

compatibly with the actions of $\mathfrak{H}^{*}$ and $\Gamma$.

Proof. Applying $e^{*}$ to the short exact sequence $H\left(X_{r} / R_{r}\right)$ yields a short exact sequence

$$
0 \longrightarrow e^{*} H^{0}\left(\omega_{r}\right) \longrightarrow e^{*} H_{\mathrm{dR}, r}^{1} \longrightarrow e^{*} H^{1}\left(\mathscr{O}_{r}\right) \longrightarrow 0
$$

of $R_{r}\left[\Delta_{1} / \Delta_{r}\right]$-modules with linear $\mathfrak{H}_{r}^{*}$-action and $R_{r}$-semilinear $\Gamma$-action in which each term is free as an $R_{r}$-module. ${ }^{10}$ Similarly, for each pair of nonnegative integers $r \geq s$, the trace mappings (3.2.4) induce a commutative diagram with exact rows

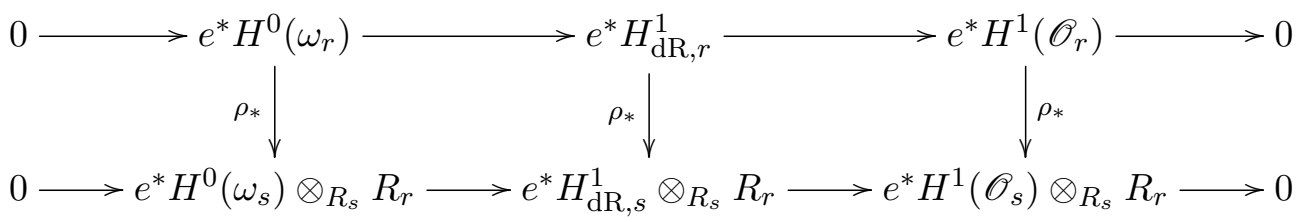

We will apply Lemma 3.1.2 with $A_{r}=R_{r}, I_{r}=\left(\varepsilon^{(r)}-1\right), B=R_{\infty}$ and with $M_{r}$ each one of the terms in (3.2.7). In order to do this, we must check that the hypotheses (3.1.1a) and (3.1.1b) are satisfied.

Applying $\otimes_{R_{r}} \mathbf{F}_{p}$ to the short exact sequence (3.2.7) and using the fact that the idempotent $e^{*}$ commutes with tensor products, we obtain, thanks to Lemma A.14 (1), the short exact sequence of

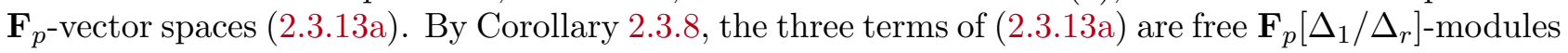

\footnotetext{
${ }^{10}$ Indeed, $e^{*} M$ is a direct summand of $M$ for any $\mathfrak{H}_{r}^{*}$-module $M$, and hence $R_{r}$-projective $\left(=R_{r}\right.$-free) if $M$ is.
} 
of ranks $d, 2 d$, and $d$ respecvitely, so (3.1.1a) is satisfied for each of these terms. Similarly, applying $\otimes_{R_{r}} \mathbf{F}_{p}$ to the diagram (3.2.8) yields a diagram which by Corollary 2.3.7 is naturally isomorphic to the diagram of $\mathbf{F}_{p}\left[\Delta_{1} / \Delta_{r}\right]$-modules with split-exact rows

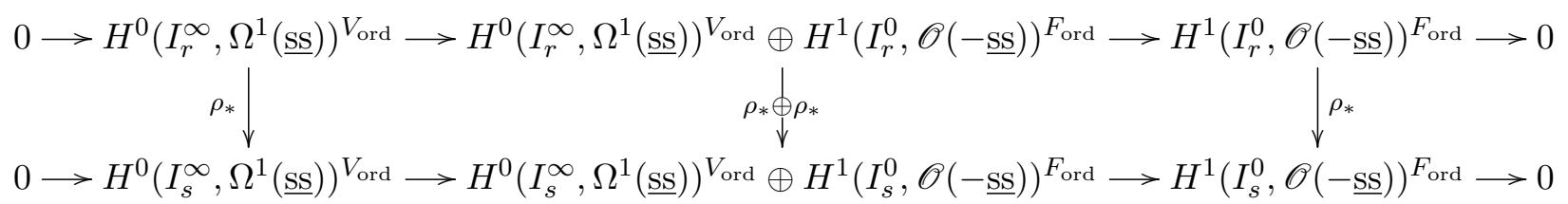

Each of the vertical maps in this diagram is surjective due to Proposition 2.2 .1 (2), and we conclude that the hypothesis (3.1.1b) is satisfied as well. Furthermore, the vertical maps in (3.2.8) are then surjective by Nakayama's Lemma, so applying $\otimes_{R_{r}} R_{\infty}$ yields an inverse system of short exact sequences in which the first term satisfies the Mittag-Leffler condition. Passing to inverse limits is therefore (right) exact, and we obtain the short exact sequence (3.2.5).

Due to Proposition A.10 (3), the short exact sequence (3.2.2) is auto-dual with respect to the canonical cup-product pairing $(\cdot, \cdot)_{r}$ on $H_{\mathrm{dR}, r}^{1}$. Extending scalars along $R_{r} \rightarrow R_{r}^{\prime}:=R_{r}\left[\mu_{N}\right]$, the Atkin-Lehner "involution" $w_{r}$ is defined on $X_{r} \times_{R_{r}} R_{r}^{\prime}$ immediately above Propisition B.9, and induces an automorphism of $\left(e^{*} H_{\mathrm{dR}, r}^{1}\right)_{R_{r}^{\prime}}$ again denoted $w_{r}$. We consider the "twisted" pairing

$$
\langle\cdot, \cdot\rangle_{r}:\left(e^{*} H_{\mathrm{dR}, r}^{1}\right)_{R_{r}^{\prime}} \times\left(e^{*} H_{\mathrm{dR}, r}^{1}\right)_{R_{r}^{\prime}} \longrightarrow R_{r}^{\prime} \quad \text { given by } \quad\langle x, y\rangle_{r}:=\left(x, w_{r} U_{p}^{* r} y\right) .
$$

It is again perfect and satisfies $\left\langle T^{*} x, y\right\rangle_{r}=\left\langle x, T^{*} y\right\rangle_{r}$ for all $x, y \in\left(e^{*} H_{\mathrm{dR}, r}^{1}\right)_{R_{r}^{\prime}}$ and $T^{*} \in \mathfrak{H}_{r}^{*}$.

Proposition 3.2.4. The pairings (3.2.9) compile to give a perfect $\Lambda_{R_{\infty}^{\prime}}$-linear duality pairing

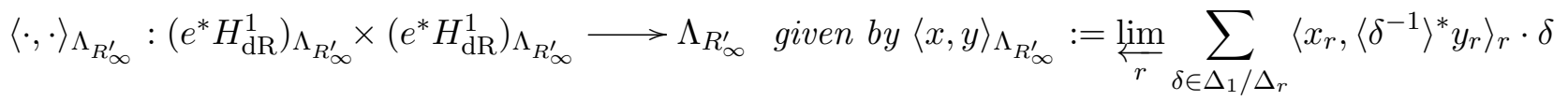

for $x=\left\{x_{r}\right\}_{r}$ and $y=\left\{y_{r}\right\}_{r}$ in $\left(e^{*} H_{\mathrm{dR}}^{1}\right)_{\Lambda_{R_{\infty}^{\prime}}}$. The pairing $\langle\cdot, \cdot\rangle_{\Lambda_{R_{\infty}^{\prime}}^{\prime}}$ induces a canonical isomorphism

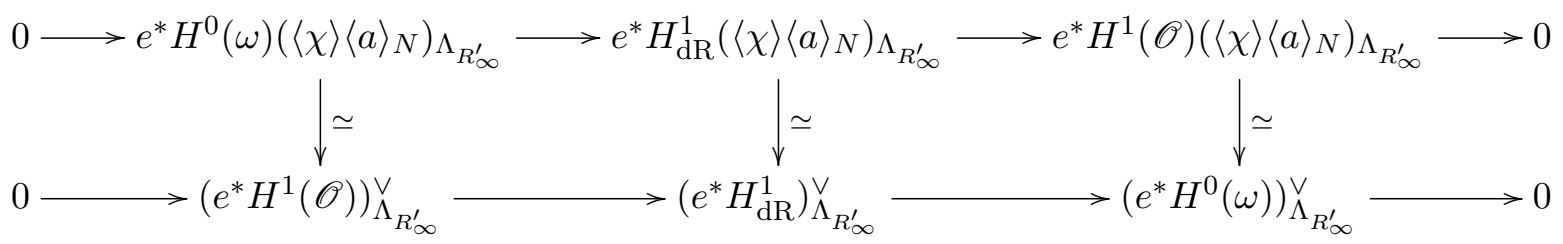

that is $\mathfrak{H}^{*}$ and $(\mathbf{Z} / N \mathbf{Z})^{\star}$-equivariant and compatible with the natural action of $\Gamma \times \operatorname{Gal}\left(K_{0}^{\prime} / K_{0}\right) \simeq$ $\operatorname{Gal}\left(K_{\infty}^{\prime} / K_{0}\right)$ on the bottom row and the twist $\gamma \cdot m:=\langle\chi(\gamma)\rangle\langle a(\gamma)\rangle_{N} \gamma m$ of the natural action on the top, where $a(\gamma) \in(\mathbf{Z} / N \mathbf{Z})^{\times}$is determined by $\zeta^{a(\gamma)}=\gamma \zeta$ for every $\zeta \in \mu_{N}\left(\overline{\mathbf{Q}}_{p}\right)$.

Proof of Proposition 3.2.4. That $\langle\cdot, \cdot\rangle_{\Lambda_{R_{\infty}^{\prime}}}$ is a perfect duality pairing follows easily from Lemma 3.1.4, using Theorem 3.2.3 and the formalism of $\S 3.1$, once we check that the twisted pairings (3.2.9) satisfy the hypothesis (3.1.3). By the definition (3.2.9) of $\langle\cdot, \cdot\rangle_{r}$, this amounts to the computation

$$
\begin{aligned}
\left(\rho_{*} x, w_{r} U_{p}^{* r} \rho_{*} y\right)_{r}=\left(x, \rho^{*} w_{r} U_{p}^{* r} \rho_{*} y\right)_{r+1} & =\left(x, w_{r+1} U_{p}^{* r} \sigma^{*} \rho_{*} y\right)_{r+1} \\
& =\sum_{\delta \in \Delta_{r} / \Delta_{r+1}}\left(x, w_{r+1} U_{p}^{* r+1}\left\langle\delta^{-1}\right\rangle^{*} y\right)_{r+1}
\end{aligned}
$$


where we have used Proposition B.9 and the identity $\sigma^{*} \rho_{*}=U_{p}^{*} \sum_{\delta \in \Delta_{r} / \Delta_{r+1}}\left\langle\delta^{-1}\right\rangle^{*}$ on $H_{\mathrm{dR}, r+1}^{1}$, which follows from ${ }^{11}$ Lemma 3.1.1, Lemma 3.1.5 and Proposition 2.2.5 of [Cais3]. We obtain an isomorphism of short exact sequences of $\Lambda_{R_{\infty}^{\prime}}$-modules as in (3.2.4), which it remains to show is $\Gamma \times \operatorname{Gal}\left(K_{0}^{\prime} / K_{0}\right)$ equivariant for the specified actions. For this, we compute that for $\gamma \in \operatorname{Gal}\left(K_{\infty}^{\prime} / K_{0}\right)$,

$$
\langle\gamma x, \gamma y\rangle_{r}=\left(\gamma x, w_{r} U_{p}^{* r} \gamma y\right)_{r}=\left(\gamma x, \gamma w_{r} U_{p}^{* r}\left\langle\chi(\gamma)^{-1}\right\rangle\left\langle a(\gamma)^{-1}\right\rangle_{N} y\right)_{r}=\gamma\left\langle x,\left\langle\chi(\gamma)^{-1}\right\rangle\left\langle a(\gamma)^{-1}\right\rangle_{N} y\right\rangle_{r},
$$

where we have used Proposition B.9 and the fact that the cup product is Galois-equivariant. It now follows easily from definitions that

$$
\langle\gamma x, \gamma y\rangle_{\Lambda_{R_{\infty}^{\prime}}}=\left\langle\chi(\gamma)^{-1}\right\rangle \gamma\left\langle x,\left\langle a(\gamma)^{-1}\right\rangle_{N} y\right\rangle_{\Lambda_{R_{\infty}^{\prime}}}
$$

and the claimed $\Gamma \times \operatorname{Gal}\left(K_{0}^{\prime} / K_{0}\right)$-equivariance of (3.2.4) is equivalent to this.

Remark 3.2.5. For an open subgroup $H$ of $\mathscr{G}_{K}$ and any $H$-stable subfield $F$ of $\mathbf{C}_{K}$, denote by $\operatorname{Rep}_{F}(H)$ the category of finite-dimensional $F$-vector spaces that are equipped with a continuous semilinear action of $H$. Recall [Sen] that classical Sen theory provides a functor $\mathbf{D}_{\text {Sen }}: \operatorname{Rep}_{\mathbf{C}_{K}}\left(\mathscr{G}_{K}\right) \rightarrow \operatorname{Rep}_{K_{\infty}}(\Gamma)$ which is quasi-inverse to $(\cdot) \otimes_{K_{\infty}} \mathbf{C}_{K}$. Furthermore, for any $W \in \operatorname{Rep}_{\mathbf{C}_{K}}\left(\mathscr{G}_{K}\right)$, there is a unique $K_{\infty}$-linear operator $\Theta_{D}$ on $D:=\mathbf{D}_{\text {Sen }}(W)$ with the property that $\gamma x=\exp \left(\log \chi(\gamma) \cdot \Theta_{D}\right)(x)$ for all $x \in D$ and all $\gamma$ in a small enough open neighborhood of $1 \in \Gamma$.

We expect that for $W$ any specialization of $e^{*} H_{\text {ét }}^{1}$ along a continuous homomorphism $\Lambda \rightarrow K_{\infty}$, there is a canonical isomorphism between $D:=\mathbf{D}_{\text {Sen }}\left(W \otimes \mathbf{C}_{K}\right)$ and the corresponding specialization of $e^{*} H_{\mathrm{dR}}^{1}$, with the Sen operator $\Theta_{D}$ induced by the Gauss-Manin connections on $H_{\mathrm{dR}, r}^{1}$. In this way, we might think of $e^{*} H_{\mathrm{dR}}^{1}$ as a $\Lambda$-adic avatar of " $\mathbf{D}_{\text {Sen }}\left(e^{*} H_{\text {ét }}^{1} \otimes_{\Lambda} \Lambda_{\mathscr{C}_{\mathbf{C}_{K}}}\right)$." We hope to pursue these connections in future work.

3.3. Relation to ordinary $\Lambda$-adic modular forms. In this section, we discuss the relation between $e^{*} H^{0}(\omega)$ and ordinary $\Lambda_{R_{\infty}}$-adic cuspforms as defined by Ohta [Ohta1, Definition 2.1.1].

We begin with some preliminaries on modular forms. For a ring $A$, a congruence subgroup $\Gamma$, and a nonnegative integer $k$, we will write $S_{k}(\Gamma ; A)$ for the space of weight $k$ cuspforms for $\Gamma$ over $A$; we put $S_{k}(\Gamma):=S_{k}(\Gamma ; \overline{\mathbf{Q}})$. If $\Gamma^{\prime}, \Gamma$ are congruence subgroups and $\gamma \in \mathrm{GL}_{2}(\mathbf{Q})$ satisfies $\gamma^{-1} \Gamma^{\prime} \gamma \subseteq \Gamma$, then there is a canonical injective "pullback" map on modular forms $\iota_{\gamma}: S_{k}(\Gamma) \longleftrightarrow S_{k}\left(\Gamma^{\prime}\right)$ given by $\iota_{\gamma}(f):=\left.f\right|_{\gamma^{-1}}$. When $\Gamma^{\prime} \subseteq \Gamma$, unless specified to the contrary, we will always view $S_{k}(\Gamma)$ as a subspace of $S_{k}\left(\Gamma^{\prime}\right)$ via $\iota_{\text {id }}$. As $\gamma \Gamma^{\prime} \gamma^{-1}$ is necessarily of finite index in $\Gamma$, one also has a canonical "trace" mapping

$$
\operatorname{tr}_{\gamma}: S_{k}\left(\Gamma^{\prime}\right) \longrightarrow S_{k}(\Gamma) \quad \text { given by } \quad \operatorname{tr}_{\gamma}(f):=\left.\sum_{\delta \in \gamma^{-1} \Gamma^{\prime} \gamma \backslash \Gamma}\left(\left.f\right|_{\gamma}\right)\right|_{\delta}
$$

with the property that $\operatorname{tr}_{\gamma} \circ \iota_{\gamma}$ is multiplication by $\left[\Gamma: \gamma^{-1} \Gamma^{\prime} \gamma\right]$ on $S_{k}(\Gamma)$.

We put $\Gamma_{r}:=\Gamma_{1}\left(N p^{r}\right)$ and define

$$
S_{2}^{\infty}\left(\Gamma_{r} ; R_{r}\right):=S_{2}\left(\Gamma_{r} ; R_{r}\right) \quad \text { and } \quad S_{2}^{0}\left(\Gamma_{r} ; R_{r}\right):=\left\{f \in S_{2}\left(\Gamma_{r} ; \overline{\mathbf{Q}}_{p}\right):\left.f\right|_{w_{r}} \in S_{2}^{\infty}\left(\Gamma_{r} ; R_{r}\right)\right\} .
$$

By definition, $S_{2}^{\star}\left(\Gamma_{r} ; R_{r}\right)$ for $\star=0, \infty$ are $R_{r}$-submodules of $S_{2}\left(\Gamma_{r} ; K_{r}^{\prime}\right)$ that are carried isomorphically onto each other by the automorphism $w_{r}$ of $S_{2}\left(\Gamma_{r} ; K_{r}^{\prime}\right)$. Note that $S_{2}^{\star}\left(\Gamma_{r} ; R_{r}\right)$ is precisely the $R_{r}$-submodule consisting of cuspforms whose formal expansion at the cusp $\star$ has coefficients in $R_{r}$. As the Hecke algebra $\mathfrak{H}_{r}$ stabilizes $S_{2}^{\infty}\left(\Gamma_{r} ; R_{r}\right)$, it follows immediately from the intertwining relation $w_{r} T=T^{*} w_{r}$ for any $T \in \mathfrak{H}_{r}($ see $\S 1.4)$ that $S_{2}^{0}\left(\Gamma_{R} ; R_{r}\right)$ is stable under the action of $\mathfrak{H}_{r}^{*}$ on $S_{2}\left(\Gamma_{r} ; K_{r}\right)$.

\footnotetext{
${ }^{11}$ The reader will check that our reliance on the sequel [Cais3] of the present paper does not involve circular reasoning.
} 
Furthermore, $\operatorname{Gal}\left(K_{r}^{\prime} / K_{0}\right)$ acts on $S_{2}\left(\Gamma_{r} ; K_{r}^{\prime}\right) \simeq S_{2}\left(\Gamma_{r} ; \mathbf{Q}_{p}\right) \otimes_{\mathbf{Q}_{p}} K_{r}^{\prime}$ through the second tensor factor, and this action leaves stable the $R_{r}$-submodule $S_{2}^{\infty}\left(\Gamma_{r} ; R_{r}\right)$. The second equality of Proposition B.9 then implies that $S_{2}^{0}\left(\Gamma_{r} ; R_{r}\right)$ is also a $\operatorname{Gal}\left(K_{r}^{\prime} / K_{0}\right)$-stable $R_{r}$-submodule of $S_{2}\left(\Gamma_{r} ; K_{r}^{\prime}\right)$. A straightforward computation shows that the direct factor $\operatorname{Gal}\left(K_{0}^{\prime} / K_{0}\right)$ of $\operatorname{Gal}\left(K_{r}^{\prime} / K_{0}\right)$ acts trivially on $S_{2}^{\infty}\left(\Gamma_{r} ; R_{r}\right)$ and through $\langle a\rangle_{N}^{-1}$ on $S_{2}^{0}\left(\Gamma_{r} ; R_{r}\right)$.

We can interpret $S_{2}^{\star}\left(\Gamma_{r} ; R_{r}\right)$ geometrically as follows. As in Remark B.16, for $\star=\infty, 0$ let $I_{r}^{\star}$ be the irreducible component of $\bar{X}_{r}$ passing through the cusp $\star$, and denote by $X_{r}^{\star}$ the complement in $X_{r}$ of all irreducible components of $\bar{X}_{r}$ distinct from $I_{r}^{\star}$. By construction, $X_{r}$ and $X_{r}^{\star}$ have the same generic fiber $X_{r} \times{ }_{\mathbf{Q}_{p}} K_{r}$. Using Proposition B.14, it is not hard to show that the diamond operators induce automorphisms of $X_{r}^{\star}$, and one checks via Proposition B.18 that the "semilinear" action (B.3) of $\gamma \in \Gamma$ on $X_{r}$ carries $X_{r}^{\star}$ to $\left(X_{r}^{\star}\right)_{\gamma}$ for all $\gamma$.

Lemma 3.3.1. Formal expansion at the $R_{r}$-point $\infty$ (respectively $R_{r}^{\prime}$-point 0$)$ of $X_{r}^{\star}$ induces an isomorphism of $R_{r}$-modules

$$
H^{0}\left(X_{r}^{\infty}, \Omega_{X_{r}^{\infty} / R_{r}}^{1}\right) \simeq S_{2}^{\infty}\left(\Gamma_{r} ; R_{r}\right) \quad \text { respectively } H^{0}\left(X_{r}^{0}, \Omega_{X_{r}^{0} / R_{r}}^{1}\right)\left(\langle a\rangle_{N}^{-1}\right) \simeq S_{2}^{0}\left(\Gamma_{r} ; R_{r}\right)
$$

which is equivariant for the natural actions of $\Gamma$ and $\mathfrak{H}_{r}$ (respectively $\left.\mathfrak{H}_{r}^{*}\right)$ on source and target and, in the case of the second isomorphism, intertwines the action of $\operatorname{Gal}\left(K_{0}^{\prime} / K_{0}\right)$ via $\langle a\rangle_{N}^{-1}$ on source with the natural action on the target.

Proof. The proof is a straightforward adaptation of the proof of [Edixhoven, Proposition 2.5].

Due to Remark B.7 and Proposition B.14, the map $x_{r} \rightarrow S_{r}$ is smooth outside the supersingular points and these points lie on every irreducible component of $\bar{X}_{r}$, so it follows from the very definition of $X_{r}^{\star}$ that there is a canonical closed immersion $\iota_{r}^{\star}: X_{r}^{\star} \hookrightarrow X_{r}^{\mathrm{sm}}$. Using Lemmas A.8 and 3.3.1, pullback of differentials along $\iota_{r}^{\star}$ gives a natural map

$$
H^{0}\left(X_{r}, \omega_{X_{r} / T_{r}}\right) \simeq H^{0}\left(X_{r}^{\mathrm{sm}}, \Omega_{X_{r}^{\mathrm{sm}} / T_{r}}^{1}\right) \stackrel{\left(\iota_{r}^{\star}\right)^{*}}{\longrightarrow} H^{0}\left(X_{r}^{\star}, \Omega_{X_{r}^{\star} / T_{r}}^{1}\right) \simeq S_{2}^{\star}\left(\Gamma_{r} ; R_{r}\right)
$$

which is an isomorphism after inverting $p$ as $X_{r}^{\text {sm }}$ and $X_{r}^{\star}$ have the same generic fiber. In particular, the map (3.3.3) is injective, $\Gamma$ and $\mathfrak{H}_{r}$ (respectively $\mathfrak{H}_{r}^{*}$ ) equivariant for $\star=\infty$ (respectively $\star=0$ ), and in the case of $\star=0$ intertwines the action of $\operatorname{Gal}\left(K_{0}^{\prime} / K_{0}\right)$ via $\langle a\rangle_{N}^{-1}$ on source with the natural action on the target.

Remark 3.3.2. The image of (3.3.3) for $\star=\infty$ is naturally identified with the space of weight 2 cuspforms for $\Gamma_{r}$ whose formal expansion at every cusp has $R_{r}$-coefficients.

Applying the idempotent $e$ (respectively $\left.e^{*}\right)$ to (3.3.3) with $\star=\infty$ (respectively $\left.\star=0\right)$ gives an injective homomorphism

$$
e H^{0}\left(X_{r}, \omega_{X_{r} / T_{r}}\right) \longleftrightarrow e S_{2}^{\infty}\left(N p^{r} ; R_{r}\right)
$$

respectively

$$
e^{*} H^{0}\left(X_{r}, \omega_{X_{r} / T_{r}}\right)\left(\langle a\rangle_{N}^{-1}\right) \longleftrightarrow e^{*} S_{2}^{0}\left(N p^{r} ; R_{r}\right)
$$

which is compatible with the canonical actions of $\Gamma$ and of $\mathfrak{H}_{r}$ (respectively $\mathfrak{H}_{r}^{*}$ ) on source and target and in the case of $(3.3 .4 \mathrm{a})$ is $\operatorname{Gal}\left(K_{0}^{\prime} / K_{0}\right)$-equivariant.

Proposition 3.3.3. The mappings (3.3.4a) and (3.3.4b) are isomorphisms. 
Proof. We treat the case of (3.3.4a); the proof that (3.3.4b) is an isomorphism goes through mutatis mutandis. We must show that (3.3.4a) is surjective. To do this, let $\nu \in e_{r} S_{2}^{\infty}\left(N p^{r} ; R_{r}\right)$ be arbitrary. Since (3.3.4a) is an isomorphism after inverting $\varpi_{r}:=\varepsilon^{(r)}-1$, there exists a least nonnegative integer $d$ such that $\varpi_{r}^{d} \nu$ is in the image of (3.3.4a). Assume that $d \geq 1$, and let $\eta \in e H^{0}\left(X_{r}, \omega_{X_{r} / R_{r}}\right)$ be any element mapping to $\varpi_{r}^{d} \nu$. For an irreducible component $I$ of $\bar{X}_{r}$, write $I^{h}$ for the complement of the supersingular points in $I$, and denote by $i_{r}^{\infty}: I_{r}^{\infty, h} \hookrightarrow X_{r}^{\infty}$ the canonical immersion. We then have a commutative diagram

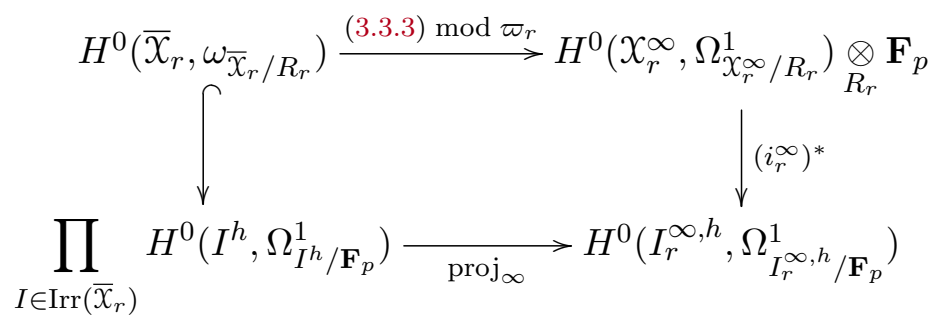

where the left vertical mapping is deduced from (2.3.3), while the bottom map is simply projection. Our assumption that $d \geq 1$ implies that the image of $\bar{\eta}:=\eta \bmod \varpi_{r}$ under the composite of the right vertical and top horizontal maps in (3.3.5) is zero and hence, viewing $\bar{\eta}=\left(\eta_{(a, b, u)}\right)$ as a meromorphic differential on the normalization of $\bar{X}_{r}$, we have $\eta_{(r, 0,1)}=\operatorname{proj}_{\infty}(\bar{\eta})=0$. Using the formula (2.3.5a), we deduce that $U_{p}^{n} \bar{\eta}=0$ for $n$ sufficiently large. But $U_{p}$ acts invertibly on $\eta$ (and hence on $\bar{\eta}$ ) so we necessarily have that $\bar{\eta}=0$ or what is the same thing that $\eta \bmod \varpi_{r}=0$. We conclude that $\varpi_{r}^{d-1} \nu$ is in the image of (3.3.4a), contradicting the minimality of $d$. Thus $d=0$ and (3.3.4a) is surjective.

For $s \leq r$, Ohta shows [Ohta1, 2.3.4] that the trace mapping $\operatorname{tr}_{\mathrm{id}}: S_{k}\left(\Gamma_{r} ; K_{r}\right) \rightarrow S_{k}\left(\Gamma_{s} ; K_{s}\right) \otimes_{K_{s}} K_{r}$ attached to the inclusion $\Gamma_{r} \subseteq \Gamma_{s}$ carries $S_{k}^{0}\left(\Gamma_{r} ; R_{r}\right)$ into $S_{k}^{0}\left(\Gamma_{s} ; R_{s}\right) \otimes_{R_{s}} R_{r}$, so that the projective limit

$$
\mathfrak{S}_{k}^{*}\left(N, R_{\infty}\right):=\lim _{\mathrm{tr}_{\mathrm{id}}}\left(S_{k}^{0}\left(\Gamma_{r} ; R_{r}\right) \otimes_{R_{r}} R_{\infty}\right)
$$

makes sense. It is canonically a $\Lambda_{R_{\infty}}$-module, equipped with an action of $\mathfrak{H}^{*}$, a semilinear action of $\Gamma$, and a natural action of $\operatorname{Gal}\left(K_{0}^{\prime} / K_{0}\right)$. On the other hand, let $e S\left(N ; \Lambda_{R_{\infty}}\right) \subseteq \Lambda_{R_{\infty}} \llbracket q \rrbracket$ be the space of ordinary $\Lambda_{R_{\infty}}$-adic cuspforms of level $N$, as defined in [Ohta1, 2.5.5]. This space is equipped with an action of $\mathfrak{H}$ via the usual formulae on formal $q$-expansions (see, for example [Wiles1, $\S 1.2]$ ), as well as an action of $\Gamma$ via its $q$-coefficientwise action on $\Lambda_{R_{\infty}} \llbracket q \rrbracket$.

Theorem 3.3.4 (Ohta). There is a canonical isomorphism of $\Lambda_{R_{\infty}}$-modules

$$
e S\left(N ; \Lambda_{R_{\infty}}\right)\left(\langle\chi\rangle^{-1}\langle a\rangle_{N}^{-1}\right) \stackrel{\simeq}{\longrightarrow} e^{*} \mathfrak{S}_{2}^{*}\left(N, R_{\infty}\right)
$$

that intertwines the action of $T \in \mathfrak{H}$ on the source with that of $T^{*} \in \mathfrak{H}^{*}$ on the target, for all $T \in \mathfrak{H}$. This isomorphism is $\operatorname{Gal}\left(K_{\infty}^{\prime} / K_{0}\right)$-equivariant for the natural action of $\operatorname{Gal}\left(K_{\infty}^{\prime} / K_{0}\right)$ on the target, and the twisted action $\gamma \cdot \mathscr{F}:=\langle\chi(\gamma)\rangle^{-1}\langle a(\gamma)\rangle_{N}^{-1} \gamma \mathscr{F}$ on the source.

Proof. For the definition of the canonical map (3.3.6), as well as the proof that it is an isomorphism, see Theorem 2.3.6 and its proof in [Ohta1]. With the conventions of [Ohta1], the claimed compatibility of (3.3.6) with Hecke operators is a consequence of [Ohta1, 2.5.1], while the $\operatorname{Gal}\left(K_{\infty}^{\prime} / K_{0}\right)$-equivariance of (3.3.6) follows from [Ohta1, Proposition 3.5.6]. 
Corollary 3.3.5. There is a canonical isomorphism of $\Lambda_{R_{\infty}}$-modules

$$
e S\left(N ; \Lambda_{R_{\infty}}\right)\left(\langle\chi\rangle^{-1}\right) \simeq e^{*} H^{0}(\omega)
$$

that intertwines the action of $T \in \mathfrak{H}$ on $e S\left(N ; \Lambda_{R_{\infty}}\right)$ with $T^{*} \in \mathfrak{H}^{*}$ on $e^{*} H^{0}(\omega)$, and is $\Gamma$-equivariant for the canonical action of $\Gamma$ on the target and the twisted action $\gamma \cdot \mathscr{F}:=\langle\chi(\gamma)\rangle^{-1} \gamma \mathscr{F}$ on the source.

Proof. This follows immediately from Proposition 3.3.3 and Theorem 3.3.4.

\section{APPENDICES}

In the following appendices, we recall the technical geometric background used in our constructions. Much (though not all) of this material is contained in [Cais1], [Cais2] and [KM].

A. Dualizing sheaves and cohomology. We begin by describing a certain modification of the usual de Rham complex for non-smooth curves. The hypercohomology of this (two-term) complex is in general much better behaved than algebraic de Rham cohomology and will enable us to construct our $\Lambda$-adic de Rham cohomology. We largely refer to [Cais1], but remark that our treatment here is different in some places and better suited to our purposes.

Definition A.1. A curve over a scheme $S$ is a morphism $f: X \rightarrow S$ of finite presentation which is a flat local complete intersection ${ }^{12}$ of pure relative dimension 1 with geometrically reduced fibers. We will often say that $X$ is a curve over $S$ or that $X$ is a relative $S$-curve when $f$ is clear from context.

Proposition A.2. Let $f: X \rightarrow S$ be a flat morphism of finite presentation. The following are equivalent:

(1) The morphism $f: X \rightarrow S$ is a curve.

(2) For every $s \in S$, the fiber $f_{s}: X_{s} \rightarrow \operatorname{Spec} k(s)$ is a curve.

(3) For every $x \in X$ with $s=f(x)$, the local ring $\mathscr{O}_{X_{s}, x}$ is a complete intersection ${ }^{13}$ and $f$ has geometrically reduced fibers of pure dimension 1.

Moreover, any base change of a curve is again a curve.

Proof. Since $f$ is flat and of finite presentation, the definition of local complete intersection that we are using (i.e. [SGA6, Exp. VIII, 1.1]) is equivalent to the definition given in $\left[\mathrm{EGA}, \mathrm{IV}_{4}, 19.3 .6\right]$ by [SGA6, Exp. VIII, 1.4]; the equivalence of (1)-(3) follows immediately. The final statement of the proposition is an easy consequence of $\left[\mathrm{EGA}, \mathrm{IV}_{4}, 19.3 .9\right]$.

Recall that a finite type morphism $f: X \rightarrow S$ with $S$ noetherian is said to be Gorenstein (respectively Cohen-Macaulay) if it is flat with all fibers Gorenstein (respectively Cohen-Macaulay) schemes.

Corollary A.3. Let $f: X \rightarrow S$ be a finite type morphism of pure relative dimension 1 over a noetherian scheme $S$.

(1) If $f$ is smooth, then it is a curve.

(2) If $X$ and $S$ are regular and $f$ has geometrically reduced fibers then $f$ is a curve.

(3) If $f$ is a curve then it is Gorenstein and hence also Cohen-Macaulay.

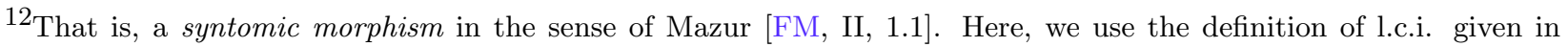
[SGA6, Exp. VIII, 1.1].

${ }^{13}$ That is, the quotient of a regular local ring by a regular sequence.
} 
Proof. The assertion (1) is obvious, and (2) follows from the fact that a closed subscheme of a regular scheme is regular if and only if it is defined (locally) by a regular sequence; $c f$. [Liu, 6.3.18]. Finally, (3) follows from Proposition A.2 (3) and the fact that every local ring that is a complete intersection is Gorenstein and hence Cohen-Macaulay (see, e.g., Theorems 18.1 and 21.3 of [Matsumura]).

Fix a relative curve $f: X \rightarrow S$, and assume that $S$ is noetherian. Since $f$ is CM by Corollary A.3 (3), thanks to Theorem 3.5.1 and the discussion immediately following 3.5.2 in [Conrad], the relative dualizing sheaf for $X$ over $S$ (or for $f$ ), denoted $\omega_{X / S}$ or $\omega_{f}$, exists. As the fibers of $f$ are Gorenstein, $\omega_{X / S}$ is an invertible $\mathscr{O}_{X}$-module by [Hartshorne, V, Proposition 9.3, Theorem 9.1]. The formation of $\omega_{X / S}$ is moreover compatible with arbitrary base change $S^{\prime} \rightarrow S$ with $S^{\prime}$ noetherian, and étale localization on $X$ [Conrad, Theorem 3.6.1]. In our situation, one moreover has a canonical $\mathscr{O}_{X}$-module homomorphism:

$$
c_{X / S}: \Omega_{X / S}^{1} \longrightarrow \omega_{X / S}
$$

constructed as in the proof of [AEZ, Théorème III.1] (see also [Liu, 6.4.13]).

Proposition A.4. Let $X \rightarrow S$ be a relative curve. The formation of the canonical map (A.1) is compatible with any base change $S^{\prime} \rightarrow S$ with $S^{\prime}$ noetherian. Moreover, the restriction of $c_{X / S}$ to the $S$-smooth locus $X^{\text {sm }}$ of $f$ in $X$ is an isomorphism.

Proof. See [AEZ], especially Théorème III.1 and $c f$. [Liu, §6.4.2].

Definition A.5. We define the two-term $\mathscr{O}_{S}$-linear complex (of $\mathscr{O}_{S}$-flat coherent $\mathscr{O}_{X}$-modules) concentrated in degrees 0 and 1

$$
\omega_{f}^{\bullet}=\omega_{X / S}^{\bullet}:=\mathscr{O}_{X} \stackrel{d_{S}}{\longrightarrow} \omega_{X / S}
$$

where $d_{S}$ is the composite of the map (A.1) and the universal $\mathscr{O}_{S}$-derivation $\mathscr{O}_{X} \rightarrow \Omega_{X / S}^{1}$. We view $\omega_{X / S}^{\bullet}$ as a filtered complex via "la filtration bête" [Deligne2], which provides an exact triangle

$$
\omega_{X / S}[-1] \longrightarrow \omega_{X / S}^{\bullet} \longrightarrow \mathscr{O}_{X}
$$

in the derived category that we call the Hodge Filtration of $\omega_{X / S}^{\bullet}$.

Since $c_{X / S}$ is an isomorphism over the $S$-smooth locus $X^{\text {sm }}$ of $f$ in $X$, the complex $\omega_{X / S}^{\bullet}$ coincides with the usual de Rham complex over $X^{\mathrm{sm}}$. Moreover, it follows immediately from Proposition A.4 that the formation of $\omega_{X / S}^{\bullet}$ is compatible with any base change $S^{\prime} \rightarrow S$ to a noetherian scheme $S^{\prime}$.

Definition A.6. Let $f: X \rightarrow S$ be a relative curve over $S$. For each nonnegative integer $i$, we define

$$
\mathscr{H}^{i}(X / S):=\mathbf{R}^{i} f_{*} \omega_{X / S}^{\bullet} .
$$

When $S=\operatorname{Spec} R$ is affine, we will write $H^{i}(X / R)$ for the global sections of the $\mathscr{O}_{S}$-module $\mathscr{H}^{i}(X / S)$.

The complex $\omega_{X / S}^{\bullet}$ and its filtration (A.3) behave extremely well with respect to duality:

Proposition A.7. Let $f: X \rightarrow S$ be a curve over a noetherian scheme $S$ and assume that $S$ is Gorenstein, excellent, and of finite Krull dimension. There is a canonical quasi-isomorphism

$$
\omega_{X / S}^{\bullet} \simeq \mathbf{R} \mathscr{H}_{o m_{X}}^{\bullet}\left(\omega_{X / S}^{\bullet}, \omega_{X / S}[-1]\right)
$$

which is compatible with the filtrations on both sides induced by (A.3). In particular: 
(1) If $f$ is proper then there is a natural quasi-isomorphism

$$
\mathbf{R} f_{*} \omega_{X / S}^{\bullet} \simeq \mathbf{R} \mathscr{H} o m_{S}^{\bullet}\left(\mathbf{R} f_{*} \omega_{X / S}^{\bullet}, \mathscr{O}_{S}\right)[-2]
$$

which is compatible with the filtrations induced by (A.3).

(2) If $\rho: Y \rightarrow X$ is any finite morphism of curves over $S$, then there is a canonical quasiisomorphism

$$
\mathbf{R} \rho_{*} \omega_{Y / S}^{\bullet} \simeq \mathbf{R} \mathscr{H} o m_{X}^{\bullet}\left(\mathbf{R} \rho_{*} \omega_{Y / S}^{\bullet}, \omega_{X / S}[-1]\right)
$$

that is compatible with filtrations.

Proof. Our hypotheses on $S$ ensure that $\mathscr{O}_{S}$ is a dualizing complex for $S$ [Hartshorne, V, $\left.\S 10\right]$, and it then follows from [Hartshorne, $\mathrm{V}, \S 8$ ] that the sheaf $\omega_{X / S}$ (thought of as a complex concentrated in some degree) is a dualizing complex for the abstract scheme $X$. To prove the first claim, we may then argue as in the proofs of Lemmas 4.3 and 5.4 of [Cais1], noting that although $S$ is assumed there to be the spectrum of a discrete valuation ring and the definition of curve in that paper differs somewhat from the definition here, the arguments themselves apply verbatim in our context. The assertion (1) (respectively (2)) follows from this by applying $\mathbf{R} f_{*}$ (respectively $\mathbf{R} \rho_{*}$ ) to both sides of (A.4) and appealing to Grothendieck duality [Conrad, Theorem 3.4.4] for the proper map $f$ (respectively $\rho$ ); see the proofs of Lemma 5.4 and Proposition 5.8 in [Cais1] for details.

In our applications, we need to understand the cohomology $H^{i}(X / S)$ for a proper curve $X \rightarrow S$ when $S$ is either the spectrum of a discrete valuation $\operatorname{ring} R$ of mixed characteristic $(0, p)$ or the spectrum of a perfect field. We now examine each of these situations in more detail.

First suppose that $S:=\operatorname{Spec}(R)$ is the spectrum of a discrete valuation ring $R$ having field of fractions $K$ of characteristic zero and residue field $k$ of characteristic $p>0$, and fix a normal curve $f: X \rightarrow S$ with smooth and geometrically connected generic fiber $X_{K}$. This situation is studied extensively in [Cais1], and we content ourselves with a summary of the results we will need. To begin, we recall the following "concrete" description of the relative dualizing sheaf:

Lemma A.8. Let $i: U \hookrightarrow X$ be any Zariski open subscheme of $X$ whose complement consists of finitely many points of codimension 2 (necessarily in the closed fiber of $X$ ). Then the canonical map

$$
\omega_{X / S} \longrightarrow i_{*} i^{*} \omega_{X / S} \simeq i_{*} \omega_{U / S}
$$

is an isomorphism. In particular, $\omega_{X / S} \simeq i_{*} \Omega_{U / S}^{1}$ for any Zariski open subscheme $i: U \hookrightarrow X^{\mathrm{sm}}$ whose complement consists of finitely many points of codimension two.

Proof. The first assertion is [Cais2, Lemma 3.2]. The second follows from this, since $X^{\text {sm }}$ contains the generic fiber and the generic points of the closed fiber by our definition of curve.

Proposition A.9. Let $\rho: Y \rightarrow X$ be a finite morphism of normal $S$-curves.

(1) Attached to $\rho$ are natural pullback and trace morphisms of complexes

$$
\rho^{*}: \omega_{X / S}^{\bullet} \longrightarrow \rho_{*} \omega_{Y / S}^{\bullet} \quad \text { and } \quad \rho_{*}: \rho_{*} \omega_{Y / S}^{\bullet} \longrightarrow \omega_{X / S}^{\bullet}
$$

which are interchanged by applying $\mathbf{R} \mathscr{H}$ om ${ }_{X}^{\bullet}\left(\cdot, \omega_{X / S}[-1]\right)$ via the duality of Proposition A.7 (2). The formation of these maps is moreover compatible with étale localization on $X$ and flat base change on $S$. 
(2) Let $U$ be any open subscheme of $X^{\mathrm{sm}}$ with the property that $V:=\rho^{-1}(U)$ is contained in $Y^{\mathrm{sm}}$. Then the induced pullback and trace mappings $\omega_{U / S}^{\bullet} \leftrightarrows \rho_{*} \omega_{V / S}^{\bullet}$ coincide with the usual pullback and trace mappings on de Rham complexes attached to the finite and flat map $\rho: V \rightarrow U$.

Proof. The assertions of (1) follow from the proofs of Propositions 4.5 and 5.5 of [Cais1], while by $S$-flatness of $X$ and $Y$, the verification of (2) reduces to checking the particular case $U=X_{K}$ and $V=Y_{K}$, which follows easily from the very definitions of $\rho_{*}$ and $\rho^{*}$ in [Cais1, $\S 4$.

We henceforth assume that the normal $S$-curve $X$ is in addition proper over $S$. Then as $X_{K}$ is a smooth and proper curve over $K$, the Hodge to de Rham spectral sequence degenerates [DI], and there is a functorial short exact sequence of $K$-vector spaces

$$
0 \longrightarrow H^{0}\left(X_{K}, \Omega_{X_{K} / K}^{1}\right) \longrightarrow H_{\mathrm{dR}}^{1}\left(X_{K} / K\right) \longrightarrow H^{1}\left(X_{K}, \mathscr{O}_{X_{K}}\right) \longrightarrow 0
$$

which we call the Hodge filtration of $H_{\mathrm{dR}}^{1}\left(X_{K} / K\right)$.

Proposition A.10. Let $f: X \rightarrow S$ be a normal curve that is proper over $S=\operatorname{Spec}(R)$.

(1) There are natural isomorphisms of free $R$-modules of rank 1

$$
H^{0}(X / R) \simeq H^{0}\left(X, \mathscr{O}_{X}\right) \text { and } H^{2}(X / R) \simeq H^{1}\left(X, \omega_{X / S}\right),
$$

which are canonically R-linearly dual to each other.

(2) There is a canonical short exact sequence of finite free $R$-modules, which we denote $H(X / R)$,

$$
0 \longrightarrow H^{0}\left(X, \omega_{X / S}\right) \longrightarrow H^{1}(X / R) \longrightarrow H^{1}\left(X, \mathscr{O}_{X}\right) \longrightarrow 0
$$

that recovers the Hodge filtration $(A .5)$ of $H_{\mathrm{dR}}^{1}\left(X_{K} / K\right)$ after extending scalars to $K$.

(3) Via the canonical cup-product auto-duality of $(A .5)$, the exact sequence $H(X / R)$ is naturally isomorphic to its R-linear dual.

(4) The exact sequence $H(X / R)$ is contravariantly (respectively covariantly) functorial in finite morphisms $\rho: Y \rightarrow X$ of normal and proper $S$-curves via pullback $\rho^{*}$ (respectively trace $\left.\rho_{*}\right)$; these morphisms recover the usual pullback and trace mappings on Hodge filtrations after extending scalars to $K$ and are adjoint with respect to the canonical cup-product autoduality of $H(X / R)$ in $(3)$.

Proof. By Raynaud's "critère de platitude cohomologique" [Raynaud, Théorme 7.2.1] (see also [Cais1, Proposition 2.7]), our requirement that curves have geometrically reduced fibers implies that $f: X \rightarrow S$ is cohomologically flat. ${ }^{14}$ The proposition now follows from Propositions 5.7-5.8 of [Cais1].

We now turn to the case that $S=\operatorname{Spec}(k)$ for a field $k$ and $f: X \rightarrow S$ is a proper and geometrically connected curve over $k$. Recall that $X$ is required to be geometrically reduced, so that the $k$-smooth locus $U:=X^{\mathrm{sm}}$ is the complement of finitely many closed points in $X$.

Proposition A.11. Let $X$ be a proper and geometrically connected curve over $k$.

(1) There are natural isomorphisms of 1-dimensional $k$-vector spaces

$$
H^{0}(X / k) \simeq H^{0}\left(X, \mathscr{O}_{X}\right) \text { and } H^{2}(X / k) \simeq H^{1}\left(X, \omega_{X / k}\right) .
$$

\footnotetext{
${ }^{14}$ In other words, the $\mathscr{O}_{S}$-module $f_{*} \mathscr{O}_{X}$ commutes with arbitrary base change.
} 
(2) There is a natural short exact sequence, which we denote $H(X / k)$

$$
0 \longrightarrow H^{0}\left(X, \omega_{X / k}\right) \longrightarrow H^{1}(X / k) \longrightarrow H^{1}\left(X, \mathscr{O}_{X}\right) \longrightarrow 0
$$

Proof. Consider the long exact cohomology sequence arising from the exact triangle (A.3). Since $X$ is proper over $k$, geometrically connected and reduced, the canonical map $k \rightarrow H^{0}\left(X, \mathscr{O}_{X}\right)$ is an isomorphism, and it follows that the map $d: H^{0}\left(X, \mathscr{O}_{X}\right) \rightarrow H^{0}\left(X, \omega_{X / k}\right)$ is zero, whence the map $H^{0}(X / k) \rightarrow H^{0}\left(X, \mathscr{O}_{X}\right)$ is an isomorphism. Thanks to Proposition A.7 (1), we have a canonical quasi-isomorphism

$$
\mathbf{R} \Gamma\left(X, \omega_{X / k}^{\bullet}\right) \simeq \mathbf{R} \operatorname{Hom}_{k}^{\bullet}\left(\mathbf{R} \Gamma\left(X, \omega_{X / k}^{\bullet}\right), k\right)[-2]
$$

that is compatible with the filtrations induced by (A.3). Using the double complex spectral sequence

$$
E_{2}^{m, n}:=\operatorname{Ext}_{k}^{m}\left(\mathbf{H}^{-n}\left(X, \omega_{X / k}^{\bullet}\right), k\right) \Longrightarrow H^{m+n}\left(\mathbf{R} \operatorname{Hom}_{k}^{\bullet}\left(\mathbf{R} \Gamma\left(X, \omega_{X / k}^{\bullet}\right), k\right)\right)
$$

and the vanishing of $\operatorname{Ext}_{k}^{m}(\cdot, k)$ for $m>0$, we deduce that $H^{2}(X / k) \simeq H^{0}(X / k)^{\vee}$ is 1-dimensional over $k$. Since Grothendieck's trace map $H^{1}\left(X, \omega_{X / k}\right) \rightarrow k$ is an isomorphism, we conclude that the surjective map of 1-dimensional $k$-vector spaces $H^{1}\left(X, \omega_{X / k}\right) \rightarrow H^{2}(X / k)$ must be an isomorphism. It follows that the map $d: H^{1}\left(X, \mathscr{O}_{X}\right) \rightarrow H^{1}\left(X, \omega_{X / k}\right)$ is zero as well, as desired.

Using the canonical map of complexes $\omega_{X / k}^{\bullet} \otimes_{\mathscr{O}_{X}} \omega_{X / k}^{\bullet} \rightarrow \omega_{X / k}^{\bullet}$, we obtain from the identification (1) of Proposition A.11 and Grothendieck's trace map a natural cup product pairing

$$
\langle\cdot, \cdot\rangle_{X}: H^{1}(X / k) \times H^{1}(X / k) \longrightarrow \mathbf{H}^{2}\left(X, \omega_{X / k}^{\bullet} \underset{\mathscr{O}_{X}}{\otimes} \omega_{X / k}^{\bullet}\right) \longrightarrow H^{2}(X / k) \simeq H^{1}\left(X, \omega_{X / k}\right) \stackrel{\operatorname{Tr}}{\longrightarrow} k
$$

under which the submodule $H^{0}\left(X, \omega_{X / k}\right)$ annihilates itself. This pairing induces a map

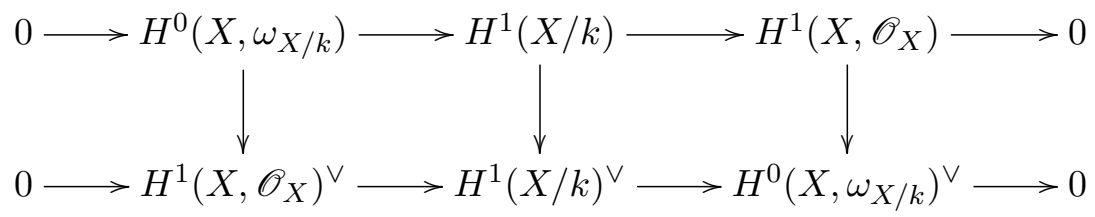

of short exact sequences of $k$-vector spaces.

Proposition A.12. The map (A.7) is an isomorphism of short exact sequences.

Proof. The flanking vertical maps are isomorphisms by [Conrad, Theorem 5.1.2], so the middle one must be as well.

When $k$ is algebraically closed, the sheaf $\omega_{X / k}$ admits a beautiful explicit description, due to Rosenlicht [Rosenlicht], in terms of meromorphic differentials on the normalization of $X$. We briefly recall this description, and refer the reader to [Conrad, §5.2] for details.

Let $k(X):=\prod_{i} k\left(\xi_{i}\right)$ be the "function field" of $X$, by definition the product of the residue fields at the finitely many generic points of $X$, and write $\Omega_{k(X) / k}^{1}$ for the pushforward of $\Omega_{k(X) / k}^{1}$ along the inclusion $\operatorname{Spec}(k(X)) \hookrightarrow X$. As $X$ is reduced, it is smooth at its generic points, so this inclusion factors through $i: X^{\mathrm{sm}} \hookrightarrow X$. By [Conrad, Lemma 5.2.1], the canonical map of $\mathscr{O}_{X}$-modules

$$
\omega_{X / k} \longrightarrow i_{*} i^{*} \omega_{X / k} \simeq i_{*} \Omega_{X}^{1}{ }^{\mathrm{sm} / k}
$$


is injective, and it follows that $\omega_{X / k}$ is a subsheaf of $\underline{\Omega}_{k(X) / k}^{1}$. Writing $\pi: X^{\mathrm{n}} \rightarrow X$ for the normalization map, we have $k(X)=k\left(X^{\mathrm{n}}\right)$ and hence an identification $\underline{\Omega}_{k(X) / k}^{1} \simeq \pi_{*} \underline{\Omega}_{k\left(X^{\mathrm{n}}\right) / k}^{1}$. For $x \in X(k)$, define

$$
\operatorname{res}_{x}: \underline{\Omega}_{k(X) / k, x}^{1} \longrightarrow k \text { by } \operatorname{res}_{x}(\eta):=\sum_{y \in \pi^{-1}(x)} \operatorname{res}_{y}(\eta),
$$

where res $_{y}$ is the classical residue map on meromorphic differentials on the smooth curve $X^{\mathrm{n}}$ over $k$.

Proposition A.13 (Rosenlicht). Let $X$ be a proper and geometrically connected curve over $k$. As a subsheaf of $\underline{\Omega}_{k(X) / k}^{1} \simeq \pi_{*} \underline{\Omega}_{k\left(X^{\mathrm{n}}\right) / k}^{1}$, the sections of $\omega_{X / k}$ over any open $V \subseteq X$ are precisely those meromorphic differentials $\eta$ on $\pi^{-1}(V) \subseteq X^{\mathrm{n}}$ that satisfy $\operatorname{res}_{x}(s \eta)=0$ for all $x \in V(k)$ and all $s \in \mathscr{O}_{X, x}$.

Proof. See [Conrad, Theorem 5.2.3].

We now suppose that $S=\operatorname{Spec}(R)$ for a discrete valuation ring $R$ with fraction field $K$ of characteristic zero and residue field $k$ of characteristic $p>0$.

Lemma A.14. Let $X$ be a normal curve over $S=\operatorname{Spec}(R)$ with smooth and geometrically connected generic fiber, and denote by $\bar{X}:=X_{k}$ the special fiber of $X$; it is a geometrically connected curve over $k$ by Proposition A.2 (2).

(1) If $X$ is proper, then the canonical base change maps

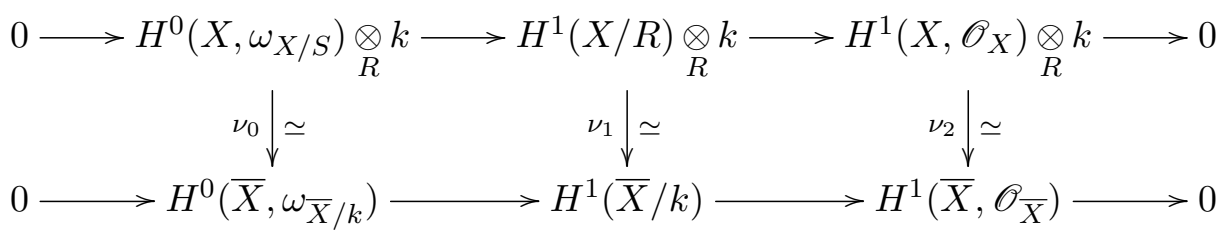

are isomorphisms, and via the auto-duality of each row one has $\nu_{i}^{\vee}=\nu_{2-i}^{-1}$ for $0 \leq i \leq 2$.

(2) Let $\rho: Y \rightarrow X$ be a finite morphism of normal curves over $S$ with smooth and geometrically connected generic fibers. The canonical diagrams (one for $\rho^{*}$ and one for $\rho_{*}$ )

$$
\begin{array}{r}
H^{0}\left(Y, \omega_{Y / S}\right) \underset{R}{\otimes} k \longrightarrow H^{0}\left(\bar{Y}, \omega_{\bar{Y} / k}\right){\underset{(A .8)}{C}}_{H^{0}\left(\bar{Y}^{\mathrm{n}}, \Omega_{k\left(\bar{Y}^{\mathrm{n}}\right) / k}^{1}\right)} \\
\rho^{*} \otimes 1||_{\rho_{*} \otimes 1} \\
\left.H^{0}\left(X, \omega_{X / S}\right) \underset{R}{\otimes} k \longrightarrow\right|_{\bar{\rho}_{*}^{\mathrm{n}}}
\end{array}
$$

commute, where $\bar{\rho}^{\mathrm{n} *}$ and $\bar{\rho}_{*}^{\mathrm{n}}$ are the usual pullback and trace morphisms on meromorphic differential forms associated to the finite flat map $\bar{\rho}^{\mathrm{n}}: \bar{Y}^{\mathrm{n}} \rightarrow \bar{X}^{\mathrm{n}}$ of smooth curves over $k$.

Proof. Since $X$ is of relative dimension 1, when it is in addition proper, the cohomologies $H^{1}\left(X, \mathscr{O}_{X}\right)$ and $H^{1}\left(X, \omega_{X / S}\right)$ both commute with base change, and are free over $R$ by Proposition A.10. In that case, we conclude that $H^{i}\left(X, \mathscr{O}_{X}\right)$ and $H^{i}\left(X, \omega_{X / S}\right)$ commute with base change for all $i$ and hence that the left and right vertical maps in the base change diagram (1) (whose rows are exact by Propositions A.10 and A.11) are isomorphisms. It follows that the middle vertical map in (1) is an isomorphism as well. Writing $\langle\cdot, \cdot\rangle_{\star}$ with $\star=X, \bar{X}$ for the canonical duality pairing on $H^{1}(X / R)$ and 
$H^{1}(\bar{X} / k)$, respectively, our assertion about the behavior of the base change isomorphisms $\nu_{i}$ under duality amounts to the equality

$$
\nu\left({\overline{\langle x, y\rangle_{X}}}\right)=\left\langle\nu_{1}(\bar{x}), \nu_{1}(\bar{y})\right\rangle_{\bar{X}}
$$

for all $x, y \in H^{1}(X / R)$; here $\overline{(\cdot)}: M \rightarrow M \otimes_{R} k$ is the natural reduction map for any $R$-module $M$ and $\nu: R \otimes_{R} k \rightarrow k$ is the canonical isomorphism. But the duality pairing is given by cup-product followed by Grothendieck's trace morphism, both of which are compatible with base change, whence the equality (A.10). The compatibility of pullback and trace under base change to the special fibers, as asserted by the diagram in (2), is a straightforward consequence of Proposition A.9 (2), using the facts that $X$ and $Y$ are smooth at generic points of closed fibers and that $\bar{\rho}: \bar{Y} \rightarrow \bar{X}$ takes generic points to generic points as noted in the proof of Lemma A.8.

We end this appendix with a brief discussion of correspondences on curves and their induced action on cohomology and Jacobians. Fix a ring $R$ and a proper curve $X$ over $S=\operatorname{Spec} R$. Throughout this discussion, we assume that $R$ is either a discrete valuation ring of mixed characteristic $(0, p)$, or a perfect field, and that $X$ is normal (and hence also smooth if $R$ is a field).

Definition A.15. A correspondence $T:=\left(\pi_{1}, \pi_{2}\right)$ on $X$ is an ordered pair $\pi_{1}, \pi_{2}: Y \rightrightarrows X$ of finite morphisms of normal and proper curves over $S$. The transpose of a correspondence $T:=\left(\pi_{1}, \pi_{2}\right)$ on $X$ is the correspondence on $X$ given by the ordered pair $T^{*}:=\left(\pi_{2}, \pi_{1}\right)$.

Thanks to Proposition A.10 (4), any correspondence $T=\left(\pi_{1}, \pi_{2}\right)$ on $X$ induces an $R$-linear endomorphism of the short exact sequence $H(X / R)$ via $\pi_{1 *} \pi_{2}^{*}$. By a slight abuse of notation, we denote this endomorphism by $T$; as endomorphisms of $H(X / R)$ we then have

$$
T=\pi_{1 *} \pi_{2}^{*} \quad \text { and } \quad T^{*}=\pi_{2 *} \pi_{1}^{*} .
$$

Given a finite map $\pi: X \rightarrow X$, we will consistently view $\pi$ as a correspondence on $X$ via the association $\pi \rightsquigarrow(\mathrm{id}, \pi)$. In this way, we may think of correspondences on $X$ as "generalized endomorphisms." This perspective can be made more compelling as follows.

Suppose that $R$ is a field, and fix a correspondence $T$ given by an ordered pair $\pi_{1}, \pi_{2}: Y \rightrightarrows X$ of finite morphisms of smooth and proper curves. Then $T$ and its transpose $T^{*}$ induce endomorphisms of the Jacobian $J_{X}:=\operatorname{Pic}_{X / R}^{0}$ of $X$, which we again denote by the same symbols, via

$$
T:=\operatorname{Alb}\left(\pi_{2}\right) \circ \operatorname{Pic}^{0}\left(\pi_{1}\right) \quad \text { and } \quad T^{*}:=\operatorname{Alb}\left(\pi_{1}\right) \circ \operatorname{Pic}^{0}\left(\pi_{2}\right) .
$$

Here, for any finite map $\pi: Y \rightarrow X$ of smooth and proper curves over a field, $\operatorname{Pic}^{0}(\pi): J_{X} \rightarrow J_{Y}$ and $\operatorname{Alb}(\pi): J_{Y} \rightarrow J_{X}$ are the maps on Jacobians functorially induced by pullback and pushforward of Weil divisors along $\pi$, respectively. Note that when $T=(\mathrm{id}, \pi)$ for a morphism $\pi: X \rightarrow X$, the induced endomorphisms (A.12) of $J_{X}$ are given by $T=\operatorname{Alb}(\pi)$ and $T^{*}:=\operatorname{Pic}^{0}(\pi) .{ }^{15}$ Abusing notation, we will simply write $\pi$ for the endomorphism $\operatorname{Alb}(\pi)$ of $J_{X}$ induced by the correspondence $(1, \pi)$, and $\pi^{*}$ for the endomorphism $\operatorname{Pic}^{0}(\pi)$ induced by $(\pi, 1)=(1, \pi)^{*}$. When $\pi: X \rightarrow X$ is an automorphism, an easy argument shows that $\pi^{*}=\pi^{-1}$ as automorphisms of $J_{X}$.

\footnotetext{
${ }^{15}$ Because of this fact, for a general correspondence $T=\left(\pi_{1}, \pi_{2}\right)$, the literature often refers to the induced endomorphism $T$ (respectively $T^{*}$ ) of $J_{X}$ as the Albanese (respectively Picard) or covariant (respectively contravariant) action of the correspondence $\left(\pi_{1}, \pi_{2}\right)$. Since the definitions (A.12) of $T$ and $T^{*}$ both literally involve Albanese and Picard functoriality, we find this old terminology confusing, and eschew it in favor of the consistent notation we have introduced.
} 
With these definitions, the canonical filtration-compatible isomorphism $H_{\mathrm{dR}}^{1}(X / R) \simeq H_{\mathrm{dR}}^{1}\left(J_{X} / R\right)$ is both $T$ and $T^{*}$-equivariant with respect to the action (A.11) on $H_{\mathrm{dR}}^{1}(X / R)$ and the action on $H_{\mathrm{dR}}^{1}\left(J_{X} / R\right)$ induced by pullback along the endomorphisms (A.12); see [Cais2, Proposition 5.4].

B. Integral models of modular curves. We record some basic facts about integral models of modular curves that will be needed in what follows. We use [KM] as our basic reference, and freely utilize the notation and terminology therein.

Throughout, we keep the notation of $\S 1.4$; in particular, recall that we have fixed a compatible sequence $\left\{\varepsilon^{(r)}\right\}_{r \geq 1}$ of primitive $p^{r}$-th roots of unity in $\overline{\mathbf{Q}}_{p}$, and that $R_{r}:=\mathbf{Z}_{p}\left[\mu_{p^{r}}\right]$ and $K_{r}:=\operatorname{Frac}\left(R_{r}\right)$. We fix a finite-type $R_{r}$-algebra $R$ that is regular and excellent, and write $\zeta \in R$ for the image of $\varepsilon^{(r)}$.

Definition B.1. The moduli problem $\left.\mathscr{P}_{r}^{\zeta}:=\left(\left[\text { bal. } \Gamma_{1}\left(p^{r}\right)\right]^{\zeta \text {-can }} ;\left[\mu_{N}\right]\right]\right)$ on $($ Ell $/ R)$ assigns to any elliptic curve $E / S$ the set of quadruples $\left(\phi: E \rightarrow E^{\prime}, P, Q ; \alpha\right)$ where:

(1) $\phi: E \rightarrow E^{\prime}$ is a cyclic $p^{r}$-isogeny.

(2) $P \in(\operatorname{ker} \phi)(S)$ and $Q \in\left(\operatorname{ker} \phi^{t}\right)(S)$ are generators of $\operatorname{ker} \phi$ and $\operatorname{ker} \phi^{t}$, respectively, which pair to $\zeta$ under the canonical pairing $\langle\cdot, \cdot\rangle_{\phi}: \operatorname{ker} \phi \times \operatorname{ker} \phi^{t} \rightarrow \mu_{\operatorname{deg} \phi}[\mathrm{KM}, \S 2.8]$.

(3) $\alpha$ is a $\mu_{N}$-structure on $E / S$, i.e. a closed immersion $\alpha: \mu_{N} \hookrightarrow E[N]$ of $S$-group schemes.

Proposition B.2. If $N \geq 4$ then $\mathscr{P}_{r}^{\zeta}$ is represented by a scheme $\mathbf{M}\left(\mathscr{P}_{r}^{\zeta}\right)$ that is flat of pure relative dimension 1 over $\operatorname{Spec}(R)$. If in addition $R$ is smooth over $R_{r}$ then $\mathbf{M}\left(\mathscr{P}_{r}^{\zeta}\right)$ is a regular scheme.

Proof. Using that $N$ is a unit in $R$, one first shows that for $N \geq 4$, the moduli problem $\left[\mu_{N}\right]$ of $\mu_{N^{-}}$ structures in the sense of $(3)$ on $(\operatorname{Ell} / R)$ is representable over $\operatorname{Spec}(R)$ and finite étale; this follows from 2.7.4, 3.6.0, 4.7.1 and 5.1.1 of $[\mathrm{KM}]$, as $\left[\mu_{N}\right]$ is isomorphic to $\left[\Gamma_{1}(N)\right]$ over any $R$-scheme containing a fixed choice of primitive $N$-th root of unity (see also [KM, 8.4.11]). By [KM, 4.3.4], it is then enough to show that $\left[\text { bal. } \Gamma_{1}\left(p^{r}\right)\right]^{\zeta \text {-can }}$ on $($ Ell $/ R)$ is relatively representable and moreover regular when $R$ is smooth over $R_{r}$, which follow from Proposition 9.1.7 and Theorem 7.6.1 (2) of [KM].

As in [KM, 8.2], for any relatively representable moduli problem $\mathscr{P}$ that is finite over $($ Ell $/ R)$, there is a canonical " $j$-line" morphism of moduli problems $\mathscr{P} \rightarrow[\Gamma(1)]$ on $($ Ell $/ R)$ that makes the coarse moduli scheme $\mathbf{M}(\mathscr{P})[\mathrm{KM}, 8.1]$ into a scheme over $\mathbf{A}_{R}^{1}:=\operatorname{Spec}(R[j])$. Using this structure map, we would like to extend $\mathbf{M}(\mathscr{P})$ to a scheme $\mathbf{M}^{\mathrm{c}}(\mathscr{P})$ over $\mathbf{P}_{R}^{1}$ by "normalizing near infinity" via the procedure described in [KM, 8.6.3]. Quite generally, assume that $Y$ is a curve over $R$ equipped with a finite morphism $Y \rightarrow \mathbf{A}_{R}^{1}:=\operatorname{Spec}(R[j])$ making $Y$ "normal near infinity" [KM, 8.6.2 C2] in the sense that there exists a monic polynomial $f \in R[j]$ with the property that $Y$ is normal over the open subscheme $U$ of $\mathbf{A}_{R}^{1}$ where $f(j) \neq 0$. Writing $D:=V(f) \subseteq \mathbf{A}_{R}^{1}$, we then denote by $Y^{\mathrm{c}}$ the glueing of $Y$ with the normalization of $U^{\mathrm{c}}:=\mathbf{P}_{R}^{1}-D$ in $\left.Y\right|_{U}$ over $\left.Y\right|_{U}$. Intrinsically, $Y^{c}$ is the unique scheme that is finite over $\mathbf{P}_{R}^{1}$, agrees with $Y$ over $\mathbf{A}_{R}^{1}$, and is normal over an open neighborhood of the $\infty$-section in $\mathbf{P}_{R}^{1}$.

Proposition B.3. If $N \geq 4$ then the compactified moduli scheme $\mathbf{M}^{\mathrm{c}}\left(\mathscr{P}_{r}^{\zeta}\right)$ is proper and flat of pure relative dimension 1 over $\operatorname{Spec}(R)$. It is moreover regular if $R$ is smooth over $R_{r}$.

Proof. This follows from [KM, 10.9.1 (2)]; see also [KM, 10.9.3] and cf. [KM, 8.6.8 (2)].

In what follows, we will frequently need to extend the map of moduli schemes $\mathbf{M}(\mathscr{P}) \rightarrow \mathbf{M}(\mathscr{Q})$ corresponding to a morphism of representable moduli problems $\mathscr{P} \rightarrow \mathscr{Q}$ that are finite over $($ Ell $/ R)$, and normal near infinity, to a morphism $\mathbf{M}^{\mathrm{c}}(\mathscr{P}) \rightarrow \mathbf{M}^{\mathrm{c}}(\mathscr{Q})$ on compactified moduli schemes. That such an extension exists is clear from the very construction of $\mathbf{M}^{\mathrm{c}}(\cdot)$ when $\mathscr{P} \rightarrow \mathscr{Q}$ is a morphism 
over $($ Ell $/ R)$, as in this case the induced mapping $\mathbf{M}(\mathscr{P}) \rightarrow \mathbf{M}(\mathscr{Q})$ is compatible with the canonical projections to the affine $j$-line by which the compactifications are built via normalization near infinity. As we will need to consider "abstract" (or exotic) morphisms $\mathscr{P} \rightarrow \mathscr{Q}[\mathrm{KM}, 11.2]$ which are not necessarily morphisms of moduli problems on $($ Ell $/ R)$, an additional argument for why the desired morphism on compactified moduli schemes exists is required. The key is the following Lemma:

Lemma B.4. Let $Y$ be a curve over $R$ equipped with two finite morphisms $\rho_{1}, \rho_{2}: Y \rightrightarrows \mathbf{A}_{R}^{1}$ such that $Y$ is normal near infinity with respect to each map, and denote by $\rho_{i}: Y_{i}^{\mathrm{c}} \rightarrow \mathbf{P}_{R}^{1}$ the compactification of $Y$ with respect to $\rho_{i}$. There is a unique isomorphism $Y_{1}^{\mathrm{c}} \simeq Y_{2}^{\mathrm{c}}$ restricting to the identity on $Y$.

Proof. By the very construction of $Y_{i}^{\mathrm{c}}$, it suffices to prove that there are open neighborhoods $V_{i}$ of $\rho_{i}^{-1}(\infty)$ and a unique isomorphism $V_{1} \simeq V_{2}$ with the property that the induced identification $V_{1} \cap Y \simeq V_{2} \cap Y$ is compatible with the inclusions $V_{i} \cap Y \hookrightarrow Y$.

We construct $V_{i}$ and such an identification $V_{1} \simeq V_{2}$ as follows. By the hypothesis that $\rho_{i}$ is normal near infinity, there is a monic polynomial $f_{i} \in R\left[x_{i}\right]$ of degree $d_{i}$ with $Y$ normal over the open $D\left(f_{i}\right)$ in $\operatorname{Spec}\left(R\left[x_{i}\right]\right)$. Let $g_{i}=x_{i}^{-d_{i}} f_{i}$, viewed as a polynomial in $R\left[x_{i}^{-1}\right]$, and denote by $W$ the scheme-theoretic image of the map $\rho_{1} \times \rho_{2}: Y \rightarrow \mathbf{A}_{R}^{1} \times_{R} \mathbf{A}_{R}^{1}=\operatorname{Spec}\left(R\left[x_{1}, x_{2}\right]\right)$. Then $W=V(F)$ for a polynomial $F\left(x_{2}, x_{2}\right)$ over $R$ which, as each $\rho_{i}$ is finite, may be taken to be monic of degree $e_{1}$ as a polynomial in $x_{1}$ over $R\left[x_{2}\right]$ and with leading term $r x_{2}^{e_{2}}$ for a unit $r \in R\left[x_{1}\right]^{\times}=R^{\times}$when viewed as a polynomial over $R\left[x_{1}\right]$. Define $h_{1}:=x_{1}^{-e_{1}} F\left(x_{1}, 0\right)$ and $h_{2}:=x_{2}^{-e_{2}} F\left(0, x_{2}\right)$ in $R\left[x_{i}^{-1}\right]\left[g_{i}^{-1}\right]$ for $i=1,2$, respectively, and set $B_{i}:=R\left[x_{i}^{-1}\right]\left[g_{i}^{-1}\right]\left[h_{i}^{-1}\right]$. It is clear from the definitions that $g_{i}$ and $h_{i}$ are units near $V\left(x_{i}^{-1}\right)$, so $\operatorname{Spec}\left(B_{i}\right)$ is an open neighborhood of the infinity section in $\mathbf{P}_{R}^{1}$. Let $A:=R\left[x_{1}, x_{2}\right] /(F)$ be the coordinate ring of $W$ and set $A^{\prime}:=A\left[\left\{x_{i}^{-1}, g_{i}^{-1}, h_{i}^{-1}\right\}_{i=1,2}\right]$, which is the coordinate ring of an open $U$ in $W$ over the infinity section of each copy of $\mathbf{P}_{R}^{1}$. Denote by $\widetilde{B}_{i}$ the normalization of $B_{i}$ in $A^{\prime}$. We claim that there are suitable localizations $C_{i}$ of $\widetilde{B}_{i}$ for $i=1,2$ with $C_{1}=C_{2}$ as subrings of the total ring of fractions of $A$. Granting this, we then take $V_{i}$ to be the normalization of $\operatorname{Spec}\left(C_{i}\right)$ in $\left.Y\right|_{U}$. By transitivity of integral closure, it is clear that $V_{i}$ is an open neighborhood of $\rho_{i}^{-1}(\infty)$ in $Y_{i}^{\mathrm{c}}$, and by construction we have the desired unique isomorphism $V_{1} \simeq V_{2}$.

To see our claim, we observe that $x_{2}^{-1}$ lies in $\widetilde{B}_{1}$. Indeed, in $A^{\prime}$ we have

$$
0=x_{1}^{-e_{1}} x_{2}^{-e_{2}} F\left(x_{1}, x_{2}\right)=x_{2}^{-e_{2}} h_{1}+\text { terms of lower degree in } x_{2}^{-1},
$$

from which it follows that $x_{2}^{-1} \in A^{\prime}$ is integral over $B_{1}=R\left[x_{1}^{-1}\right]\left[g_{1}^{-1}\right]\left[h_{1}^{-1}\right]$ so lies in $\widetilde{B}_{1}$. As $g_{2}$ and $h_{2}$ are polynomials in $x_{2}^{-1}$ with $R$-coefficients, we can then make sense of the localization $\widetilde{B}_{1}\left[g_{2}^{-1}\right]\left[h_{2}^{-1}\right]$. Now if $\alpha \in \widetilde{B}_{2}$, then $\alpha$ is integral over $B_{2}=R\left[x_{2}^{-1}\right]\left[g_{2}^{-1}\right]\left[h_{2}^{-1}\right]$, so $\left(g_{2} h_{2}\right)^{d} \alpha$ is integral over $R\left[x_{2}^{-1}\right]$ for some positive integer $d$, whence $\left(g_{2} h_{2}\right)^{d} \alpha$ lies in $\widetilde{B}_{1}$ and we conclude that $\widetilde{B}_{2} \subseteq \widetilde{B}_{1}\left[g_{2}^{-1}\right]\left[h_{2}^{-1}\right]$. Similarly, we have $\widetilde{B}_{1} \subseteq \widetilde{B}_{2}\left[g_{1}^{-1}\right]\left[h_{1}^{-1}\right]$, and it follows that as

$$
C_{1}:=\widetilde{B}_{1}\left[g_{2}^{-1}\right]\left[h_{2}^{-1}\right]=\widetilde{B}_{2}\left[g_{1}^{-1}\right]\left[h_{1}^{-1}\right]=: C_{2},
$$

as subrings of the total ring of fractions of $A$. This establishes our claim, and completes the proof.

Corollary B.5. Let $\mathscr{P} \rightarrow \mathscr{Q}$ be an abstract morphism of representable moduli problems that are finite over $($ Ell $/ R)$ and normal near infinity, and denote by $\rho: \mathbf{M}(\mathscr{P}) \rightarrow \mathbf{M}(\mathscr{Q})$ the induced morphism of moduli schemes. Assume that $\rho$ is finite and that $\mathbf{M}(\mathscr{P})$ is normal near infinity with respect to the composition $j \circ \rho$. Then there is a unique extension of $\rho$ to a finite morphism $\rho^{\mathrm{c}}: \mathbf{M}^{\mathrm{c}}(\mathscr{P}) \rightarrow \mathbf{M}^{\mathrm{c}}(\mathscr{Q})$ of the compactified moduli schemes. 
Proof. Let us write $j^{\prime}:=j \circ \rho: \mathbf{M}(\mathscr{P}) \rightarrow \mathbf{A}_{R}^{1}$ and denote by $\mathbf{M}^{\mathrm{c}}(\mathscr{P})^{\prime}$ the compactification of $M(\mathscr{P})$ using $j^{\prime}$. It follows easily from the given constructions that $\rho$ uniquely extends to a morphism $\mathbf{M}^{\mathrm{c}}(\mathscr{P})^{\prime} \rightarrow \mathbf{M}^{\mathrm{c}}(\mathscr{Q})$ that is readily checked to be finite. Applying Lemma B.4 to $Y=\mathbf{M}(\mathscr{P})$ with the two projections $j, j^{\prime}$ to $\mathbf{A}_{R}^{1}$ then gives $\mathbf{M}^{\mathrm{c}}(\mathscr{P}) \simeq \mathbf{M}^{\mathrm{c}}(\mathscr{P})^{\prime}$ uniquely, and the conclusion follows.

Definition B.6. We define $\mathcal{X}_{r}:=\mathbf{M}^{\mathrm{c}}\left(\mathscr{P}_{r}^{\varepsilon^{(r)}}\right)$, viewed as a scheme over $T_{r}:=\operatorname{Spec}\left(R_{r}\right)$.

Remark B.7. We may identify the generic fiber of $\mathcal{X}_{r}$ with $\left(X_{r}\right)_{K_{r}}$, where $X_{r}=X_{1}\left(N p^{r}\right)$ is the usual modular curve over $\mathbf{Q}$ defined as in $\$ 1.4$. Indeed, it is a standard exercise that $X_{r}$ is the compactified moduli scheme of triples $(E, \iota, \alpha)$ consisting of an elliptic curve $E$ with embeddings of group schemes $\iota: \mu_{p^{r}} \hookrightarrow E$ and $\alpha: \mu_{N} \hookrightarrow E$. Given such a triple $(E, \iota, \alpha)$ over a $K_{r}$-scheme $S$, we set $E^{\prime}:=E / \operatorname{im}(\iota)$, $P_{\iota}:=\iota\left(\varepsilon^{(r)}\right)$ and denote by $Q_{\iota} \in E^{\prime}(S)$ the unique point satisfying $\left\langle\iota(z), Q_{\iota}\right\rangle=z$ for all $z \in \mu_{p^{r}}(S)$. The association

$$
(E, \iota, \alpha) \mapsto\left(\phi: E \rightarrow E^{\prime}, P_{\iota}, Q_{\iota} ; \alpha\right)
$$

then induces the claimed identification $\left(X_{r}\right)_{K_{r}} \simeq\left(X_{r}\right)_{K_{r}}$.

There is a canonical action of $\mathbf{Z}_{p}^{\times} \times(\mathbf{Z} / N \mathbf{Z})^{\times}$by $R_{r}$-automorphisms of $X_{r}$, defined at the level of the underlying moduli problem by

$$
(u, v) \cdot\left(\phi: E \rightarrow E^{\prime}, P, Q ; \alpha\right):=\left(\phi: E \rightarrow E^{\prime}, u P, u^{-1} Q ; \alpha v\right)
$$

as one checks by means of the computation $\left\langle u P, u^{-1} Q\right\rangle_{\phi}=\langle P, Q\rangle_{\phi}^{u u^{-1}}=\langle P, Q\rangle_{\phi}$. Here, we again write $v: \mu_{N} \rightarrow \mu_{N}$ for the automorphism of $\mu_{N}$ functorially defined by $\zeta \mapsto \zeta^{v}$ for any $N$-th root of unity $\zeta$. We refer to this action of $\mathbf{Z}_{p}^{\times} \times(\mathbf{Z} / N \mathbf{Z})^{\times}$as the diamond operator action, and will denote by $\langle u\rangle$ (respectively $\langle v\rangle_{N}$ ) the automorphism induced by $u \in \mathbf{Z}_{p}^{\times}$(respectively $v \in(\mathbf{Z} / N \mathbf{Z})^{\times}$).

There is also an $R_{r}$-semilinear "geometric inertia" action of $\Gamma:=\operatorname{Gal}\left(K_{\infty} / K_{0}\right)$ on $\mathcal{X}_{r}$, which reflects the fact that the generic fiber of $X_{r}$ descends to $\mathbf{Q}_{p}$. To explain this action, for $\gamma \in \Gamma$ and any $T_{r^{-}}$ scheme $T^{\prime}$, let us write $T_{\gamma}^{\prime}$ for the base change of $T^{\prime}$ along the induced morphism $\gamma: T_{r} \rightarrow T_{r}$. For a moduli problem $\mathscr{P}$ on $\left(\right.$ Ell $\left./ T_{r}\right)$, we denote by $\gamma^{*} \mathscr{P}$ the moduli problem on $\left(\right.$ Ell $\left./\left(T_{r}\right)_{\gamma}\right)$ determined by the requirement $\left(\gamma^{*} \mathscr{P}\right)\left(E_{\gamma} / S_{\gamma}\right)=\mathscr{P}(E / S)$ for all objects $E / S$ of $\left(\right.$ Ell $\left./ T_{r}\right)$; we note that if $\mathscr{P}$ is representable, then so is $\gamma^{*} \mathscr{P}$ and we have $\mathbf{M}^{\mathrm{c}}\left(\gamma^{*} \mathscr{P}\right) \simeq \mathbf{M}^{\mathrm{c}}(\mathscr{P})_{\gamma}[\mathrm{KM}, 4.13]$ and $c f$. [KM, 12.10.1] and [Ulmer, §4]. Each $\gamma \in \Gamma$ gives rise to a morphism of moduli problems $\gamma: \mathscr{P}_{r}^{\varepsilon^{(r)}} \rightarrow \gamma^{*} \mathscr{P}_{r}^{\varepsilon^{(r)}}$ via the assignment $\gamma: \mathscr{P}(E / S) \rightsquigarrow\left(\gamma^{*} \mathscr{P}\right)(E / S)=\mathscr{P}\left(E_{\gamma^{-1}} / S_{\gamma^{-1}}\right)$ determined by

$$
\gamma\left(\phi: E \rightarrow E^{\prime}, P, Q ; \alpha\right):=\left(\phi_{\gamma^{-1}}: E_{\gamma^{-1}} \rightarrow E_{\gamma^{-1}}^{\prime}, \chi(\gamma) P_{\gamma^{-1}}, Q_{\gamma^{-1}} ; \alpha_{\gamma^{-1}}\right)
$$

where the subscript of $\gamma^{-1}$ means "base change along $\gamma^{-1}$ " (see $\S 1.4$ ). Since

$$
\left\langle\chi(\gamma) P_{\gamma^{-1}}, Q_{\gamma^{-1}}\right\rangle_{\phi_{\gamma^{-1}}}=\gamma^{-1}\langle P, Q\rangle_{\phi}^{\chi(\gamma)}=\langle P, Q\rangle_{\phi},
$$

this really is a morphism of moduli problems on $\left(\mathrm{Ell} / T_{r}\right)$, so by the discussion preceding Lemma B.4 and [KM, Proposition 9.3.1] induces a morphism of $T_{r}$-schemes

$$
\gamma: X_{r} \longrightarrow\left(X_{r}\right)_{\gamma}
$$

for each $\gamma \in \Gamma$, compatibly with change in $\gamma$.

Recall ([KM, §6.7]) that over any base scheme $S$, a cyclic $p^{r+1}$-isogeny of elliptic curves $\phi: E \rightarrow E^{\prime}$ admits a "standard factorization" (in the sense of [KM, 6.7.7])

$$
E=: E_{0} \stackrel{\phi_{0,1}}{\longrightarrow} E_{1} \cdots \longrightarrow E_{r} \stackrel{\phi_{r, r+1}}{\longrightarrow} E_{r+1}:=E^{\prime} .
$$


For each pair of nonnegative integers $a<b \leq r+1$ we will write $\phi_{a, b}$ for the composite $\phi_{b-1, b} \circ \cdots \circ \phi_{a, a+1}$ and $\phi_{b, a}:=\phi_{a, b}^{t}$ for the dual isogeny. Using this notion and appealing to Corollary B.5, we may define "degeneracy maps" $\rho, \sigma: X_{r+1} \rightrightarrows X_{r}$ (over the map $T_{r+1} \rightarrow T_{r}$ ) at the level of underlying moduli problems as follows (cf:: $[\mathrm{KM}, 11.3 .3])$ :

$$
\begin{aligned}
\rho\left(\phi: E_{0} \rightarrow E_{r+1}, P, Q ; \alpha\right) & :=\left(\phi_{0, r}: E_{0} \rightarrow E_{r}, p P, \phi_{r+1, r}(Q) ; \alpha\right) \\
\sigma\left(\phi: E_{0} \rightarrow E_{r+1}, P, Q ; \alpha\right) & :=\left(\phi_{1, r+1}: E_{1} \rightarrow E_{r+1}, \phi_{0,1}(P), p Q ; \phi_{0,1} \circ \alpha\right)
\end{aligned}
$$

By the universal property of fiber products, we obtain morphisms of $T_{r+1}$-schemes

$$
x_{r+1} \underset{\sigma}{\stackrel{\rho}{\rightleftarrows}} X_{r} \times_{T_{r}} T_{r+1}
$$

that are compatible with the diamond operators and the geometric inertia action of $\Gamma$.

Remark B.8. Via the identification of Remark B.7, the maps on generic fibers induced by (B.6) coincide with the (base change to $K_{r+1}$ of the) degeneracy maps $\rho, \sigma: X_{r+1} \rightrightarrows X_{r}$ of $\S 1.4$, which (on complex analytifications) arise from the mappings $\rho: \tau \mapsto \tau$ and $\sigma: \tau \mapsto p \tau$ on the complex upper half-plane.

Recall that we have fixed a choice of primitive $N$-th root of unity $\zeta_{N}$ in $\overline{\mathbf{Q}}_{p}$. The Atkin-Lehner "involution" $w_{\zeta_{N}}$ is defined as follows. Let $E$ be an elliptic curve over an $R_{r}^{\prime}:=R_{r}\left[\mu_{N}\right]$-scheme $S$ and $\alpha: \mu_{N} \hookrightarrow E$ an embedding of group schemes. Writing $\psi: E \rightarrow E / \operatorname{im}(\alpha)$ and $\psi^{\prime}: E^{\prime} \rightarrow E^{\prime} / \operatorname{im}(\phi \circ \alpha)$ for the canonical maps, as $N$ is a unit in $R_{r}^{\prime}$ there is a unique embedding $\beta: \mu_{N} \rightarrow E / \operatorname{im}(\alpha)$ of group schemes over $S$ satisfying $\left\langle\alpha(z), \beta\left(\zeta_{N}\right)\right\rangle_{\psi}=z$ for all $z \in \mu_{N}(S)$. Define

$$
w_{\zeta_{N}}\left(\phi: E \rightarrow E^{\prime}, P, Q ; \alpha\right):=\left(\bar{\phi}: E / \operatorname{im}(\alpha) \rightarrow E^{\prime} / \operatorname{im}(\phi \circ \alpha), \psi(P), N^{-1} \psi^{\prime}(Q) ; \beta\right),
$$

where $\bar{\phi}$ is the isogeny induced by $\phi$, and by a slight abuse we write " $N^{-1}$ " for the multiplicative inverse of $N$ in $\mathbf{Z}_{p}$. Using the basic compatibility properties of the Weil pairing we calculate

$$
\begin{aligned}
\left\langle\psi(P), N^{-1} \psi^{\prime}(Q)\right\rangle_{\bar{\phi}} & =\left\langle P, N^{-1} \psi^{\prime}(Q)\right\rangle_{\psi \bar{\phi}}=\left\langle P, N^{-1} \psi^{\prime}(Q)\right\rangle_{\psi^{\prime} \phi}=\left\langle N^{-1} \psi^{\prime}(Q), P\right\rangle_{\phi^{t} \psi^{\prime t}}^{-1} \\
& =\left\langle N^{-1} \psi^{\prime t} \psi^{\prime}(Q), P\right\rangle_{\phi^{t}}^{-1}=\langle Q, P\rangle_{\phi^{t}}^{-1}=\langle P, Q\rangle_{\phi}=\varepsilon^{(r)}
\end{aligned}
$$

so that this really does define an endomorphism of $\mathscr{P}_{r}^{\varepsilon^{(r)}}$. Invoking Corollary B.5 then gives a self-map of $X_{r} \times_{R_{r}} R_{r}^{\prime}$ over $R_{r}^{\prime}$ that we again denote by $w_{\zeta_{N}}$. Exactly as in (B.2), each $g \in \operatorname{Gal}\left(K_{0}^{\prime} / K_{0}\right)$ induces a morphism $X_{r} \times_{R_{r}} R_{r}^{\prime} \rightarrow g^{*}\left(\mathcal{X}_{r} \times_{R_{r}} R_{r}^{\prime}\right)$.

Following [KM, 11.3.2] and once again employing Corollary B.5, we define the Atkin-Lehner automorphism $w_{\varepsilon^{(r)}}$ of $X_{r}$ over $R_{r}$ at the level of underlying moduli problem by

$$
w_{\varepsilon^{(r)}}\left(\phi: E \rightarrow E^{\prime}, P, Q ; \alpha\right):=\left(\phi^{t}: E^{\prime} \rightarrow E,-Q, P ; \phi \circ \alpha\right)
$$

We then define $w_{r}:=w_{\varepsilon^{(r)}} \circ w_{\zeta_{N}}$; it is an automorphism of $X_{r} \times_{R_{r}} R_{r}^{\prime}$ over $R_{r}^{\prime}$.

Proposition B.9. $\ \triangleright \triangleright$ Bryden: [This has issues with indices: for example, $\rho w_{r}=\langle p\rangle_{N} w_{r} \sigma$ should be $\left.\rho w_{r+1}=\langle p\rangle_{N} w_{r} \sigma\right]$ 
For all $(u, v) \in \mathbf{Z}_{p}^{\times} \times(\mathbf{Z} / N \mathbf{Z})^{\times}$and all $(\gamma, g) \in \Gamma \times \operatorname{Gal}\left(K_{0}^{\prime} / K_{r}\right) \simeq \operatorname{Gal}\left(K_{\infty}^{\prime} / K_{0}\right)$, the identities

$$
\begin{aligned}
& w_{\zeta_{N}}^{2}=\langle-1\rangle_{N}\langle N\rangle \\
& w_{\varepsilon^{(r)}}^{2}=\left\langle p^{r}\right\rangle_{N}\langle-1\rangle \\
& w_{\zeta_{N}} \circ w_{\varepsilon^{(r)}}=\left\langle p^{r}\right\rangle_{N}^{-1}\langle N\rangle w_{\varepsilon^{(r)}} \circ w_{\zeta_{N}} \\
& w_{r}^{2}=\langle-1\rangle_{N}\langle-1\rangle \\
& w_{\varepsilon^{(r)}}\langle u\rangle\langle v\rangle_{N}=\left\langle u^{-1}\right\rangle\langle v\rangle_{N} w_{\varepsilon^{(r)}} \\
& w_{\zeta_{N}}\langle u\rangle\langle v\rangle_{N}=\langle u\rangle\left\langle v^{-1}\right\rangle_{N} w_{\zeta_{N}} \\
& w_{r}\langle u\rangle\langle v\rangle_{N}=\left\langle u^{-1}\right\rangle\left\langle v^{-1}\right\rangle_{N} w_{r} \\
& \rho w_{\varepsilon(r+1)}=w_{\varepsilon^{(r)}} \sigma \\
& \sigma w_{\varepsilon^{(r+1)}}=\langle p\rangle_{N} w_{\varepsilon^{(r)}} \rho \\
& \rho w_{\zeta_{N}}=w_{\zeta_{N}} \rho \\
& \sigma w_{\zeta_{N}}=\langle p\rangle_{N} w_{\zeta_{N}} \sigma \\
& \rho w_{r}=\langle p\rangle_{N} w_{r} \sigma \\
& \sigma w_{r}=\langle p\rangle_{N} w_{r} \rho \\
& \left(\gamma^{*} w_{\varepsilon^{(r)}}\right) \gamma=\left\langle\chi(\gamma)^{-1}\right\rangle \gamma w_{\varepsilon^{(r)}} \\
& \left(g^{*} w_{\zeta_{N}}\right) g=\left\langle a(g)^{-1}\right\rangle_{N} g w_{\zeta_{N}} \\
& \left((\gamma, g)^{*} w_{r}\right)(\gamma, g)=\left\langle\chi(\gamma)^{-1}\right\rangle\left\langle a(g)^{-1}\right\rangle_{N}(\gamma, g) w_{r}
\end{aligned}
$$

hold, with $a: \operatorname{Gal}\left(K_{\infty}^{\prime} / K_{0}\right) \rightarrow(\mathbf{Z} / N \mathbf{Z})^{\times}$the character determined by $\gamma \zeta=\zeta^{a(\gamma)}$ for all $\zeta \in \mu_{N}\left(\overline{\mathbf{Q}}_{p}\right)$.

Proof. This is a straightforward consequence of definitions; i.e. computing with moduli.

In order to describe the special fiber of $X_{r}$, we must first introduce Igusa curves:

Definition B.10. Let $r$ be a nonnegative integer. The moduli problem $\mathscr{I}_{r}:=\left(\left[\operatorname{Ig}\left(p^{r}\right)\right] ;\left[\mu_{N}\right]\right)$ on $\left(\right.$ Ell $\left./ \mathbf{F}_{p}\right)$ assigns to any elliptic curve $E / S$ the set of triples $(E, P ; \alpha)$ where

(1) $P \in E^{\left(p^{r}\right)}(S)$ generates the kernel of the $r$-fold iterate of Verscheibung $V^{(r)}: E^{\left(p^{r}\right)} \rightarrow E$.

(2) $\alpha: \mu_{N} \hookrightarrow E[N]$ is a closed immersion of $S$-group schemes.

The data given in (1) above is called an Igusa structure of level $p^{r}$ on $E / S$.

Proposition B.11. If $N \geq 4$, then the moduli problem $\mathscr{I}_{r}$ on $\left(\right.$ Ell $\left./ \mathbf{F}_{p}\right)$ is represented by a smooth affine curve $\mathbf{M}\left(\mathscr{I}_{r}\right)$ over $\mathbf{F}_{p}$.

Proof. One argues as in the proof of Proposition B.2, using [KM, 12.6.1] to know that $\left[\operatorname{Ig}\left(p^{r}\right)\right]$ is relatively representable on $\left(\right.$ Ell $\left./ \mathbf{F}_{p}\right)$, regular 1-dimensional and finite flat over $\left(\mathrm{Ell} / \mathbf{F}_{p}\right)$.

Definition B.12. We write $\operatorname{Ig}_{r}:=\mathbf{M}^{\mathrm{c}}\left(\mathscr{I}_{r}\right)$ for the compactified moduli scheme.

As $\operatorname{Ig}_{r}$ is normal and 1-dimensional by the very construction of $\mathbf{M}^{\mathrm{c}}(\cdot)$, it follows that $\operatorname{Ig}_{r}$ is a smooth and proper $\mathbf{F}_{p}$-curve. There is a canonical action of the diamond operators $\mathbf{Z}_{p}^{\times} \times(\mathbf{Z} / N \mathbf{Z})^{\times}$on $\mathscr{I}_{r}$ via $(u, v) \cdot(E, P ; \alpha):=\left(E, u^{-1} P ; \alpha v\right)$; this induces a corresponding action on $\operatorname{Ig}_{r}$ by $\mathbf{F}_{p^{-}}$-automorphisms. We again write $\langle u\rangle$ (respectively $\langle v\rangle_{N}$ ) for the action of $u \in \mathbf{Z}_{p}^{\times}$(respectively $v \in(\mathbf{Z} / N \mathbf{Z})^{\times}$). 
Remark B.13. The astute reader will wonder why we have defined the action of $\langle u\rangle$ on the moduli problem $\mathscr{I}_{r}$ to be $(E, P, \alpha) \rightsquigarrow\left(E, u^{-1} P, \alpha\right)$ rather than $(E, P, \alpha) \rightsquigarrow(E, u P, \alpha)$. The reason is this: in the literature (e.g [Gross, §5], [MW3], [Saby, §2.1]), one works instead with the moduli problem $\mathscr{I}_{r}^{\mu}$ classifying $(E, \iota, \alpha)$, where $\alpha$ is as above and $\iota: \mu_{p^{r}} \hookrightarrow E\left[p^{r}\right]$ is a closed immersion of $S$-group schemes. On the category of ordinary $E / S$, there is an isomorphism of moduli problems $\mathscr{I}_{r} \rightarrow \mathscr{I}_{r}^{\mu}$ given as follows: a triple $(E, P, \alpha)$ determines an isomorphism $\psi_{P}: \mathbf{Z} / p^{r} \mathbf{Z} \rightarrow \operatorname{ker}\left(V^{r}: E^{\left(p^{r}\right)} \rightarrow E\right)$, the Cartier dual of which is an isomorphism $\psi_{P}^{t}: \operatorname{ker}\left(F^{r}: E \rightarrow E^{\left(p^{r}\right)}\right) \rightarrow \mu_{p^{r}}$. The inverse of this isomorphism gives an embedding $\iota:=\left(\psi_{P}^{t}\right)^{-1}: \mu_{p^{r}} \hookrightarrow E\left[p^{r}\right]$, which gives an element $(E, \iota, \alpha)$ of $\mathscr{I}_{r}^{\mu}(E / S)$, and this association induces the claimed isomorphism of moduli problems. Under this identification, the action of $\langle u\rangle$ on $\mathscr{I}_{r}$ that we have defined is carried to the action of $\langle u\rangle$ on $\mathscr{I}_{r}^{\mu}$ given by $\langle u\rangle:(E, \iota, \alpha) \mapsto(E, u \circ \iota, \alpha)$, which agrees with the diamond operator action in loc. cit.

Thanks to the "backing up theorem" [KM, 6.7.11], one also has natural degeneracy maps

$$
\rho: \operatorname{Ig}_{r+1} \longrightarrow \operatorname{Ig}_{r} \quad \text { induced by } \quad \rho(E, P ; \alpha):=(E, V P, \alpha)
$$

on underlying moduli problems. These maps are visibly equivariant for the diamond operator action on source and target. Let $\underline{\mathrm{ss}}_{r}$ be the (reduced) closed subscheme of $\operatorname{Ig}_{r}$ that is the support of the coherent ideal sheaf of relative differentials $\Omega_{\mathrm{Ig}_{r} / \mathrm{Ig}_{0}}^{1}$; over the unique degree 2 extension of $\mathbf{F}_{p}$, this scheme breaks up as a disjoint union of rational points - the supersingular points. The map (B.7) is finite of degree $p$, generically étale and totally (wildly) ramified over each supersingular point.

We can now describe the special fiber of $X_{r}$ :

Proposition B.14. The scheme $\bar{X}_{r}:=X_{r} \times_{T_{r}} \operatorname{Spec}\left(\mathbf{F}_{p}\right)$ is the disjoint union, with crossings at the supersingular points, of the following proper, smooth $\mathbf{F}_{p}$-curves: for each pair $a, b$ of nonnegative integers with $a+b=r$, and for each $u \in\left(\mathbf{Z} / p^{\min (a, b)} \mathbf{Z}\right)^{\times}$, one copy of $\operatorname{Ig}_{\max (a, b)}$.

We refer to [KM, 13.1.5] for the definition of "disjoint union with crossings at the supersingular points". Note that the special fiber of $X_{r}$ is (geometrically) reduced; this will be crucial in our later work. We often write $I_{(a, b, u)}$ for the irreducible component of $\bar{X}_{r}$ indexed by the triple $(a, b, u)$ and will refer to it as the $(a, b, u)$-component (for fixed $(a, b)$ we have $I_{(a, b, u)}=\operatorname{Ig}_{\max (a, b)}$ for all $u$ ).

For the proof of Proposition B.14, we refer the reader to [KM, 13.11.2-13.11.4], and content ourselves with recalling the correspondence between (non-cuspidal) points of the $(a, b, u)$-component and ([bal. $\left.\left.\Gamma_{1}\left(p^{r}\right)\right]^{1-c a n} ;\left[\mu_{N}\right]\right)$-structures on elliptic curves.

Let $S$ be any $\mathbf{F}_{p^{-}}$-scheme, fix an ordinary elliptic curve $E_{0}$ over $S$, and let $\left(\phi: E_{0} \rightarrow E_{r}, P, Q ; \alpha\right)$ be an element of $\mathscr{P}_{r}^{1}\left(E_{0} / S\right)$. By [KM, 13.11.2], there exist unique nonnegative integers $a, b$ with the property that the cyclic $p^{r}$-isogeny $\phi$ factors as a purely inseparable cyclic $p^{a}$-isogeny followed by an étale $p^{b}$-isogeny (this $i s$ the standard factorization of $\phi$ ). Furthermore, there exists a unique elliptic curve $E$ over $S$ and $S$-isomorphisms $E_{0} \simeq E^{\left(p^{b}\right)}$ and $E_{r} \simeq E^{\left(p^{a}\right)}$ such that the cyclic $p^{r}$-isogeny $\phi$ is:

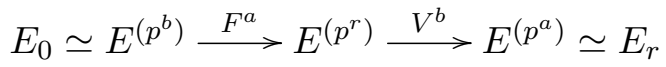

and $P \in E^{\left(p^{b}\right)}(S)$ (respectively $Q \in E^{\left(p^{a}\right)}(S)$ ) is an Igusa structure of level $p^{b}$ (respectively $p^{a}$ ) on $E$ over $S$. When $a \geq b$ there is a unique unit $u \in\left(\mathbf{Z} / p^{b} \mathbf{Z}\right)^{\times}$such that $V^{a-b}(Q)=u P$ in $E^{\left(p^{b}\right)}(S)$ and when $b \geq a$ there is a unique unit $u \in\left(\mathbf{Z} / p^{a} \mathbf{Z}\right)^{\times}$such that $u V^{b-a}(P)=Q$ in $E^{\left(p^{a}\right)}(S)$. Thus, for $a \geq b$ (respectively $b \geq a)$ and fixed $u$, the data $\left(E, Q ; V^{b} \alpha p^{-b}\right)$ (respectively $\left(E, P ; V^{b} \alpha p^{-b}\right)$ ) gives an $S$-point of the $(a, b, u)$-component $\operatorname{Ig}_{\max (a, b)}$. Conversely, suppose given $(a, b, u)$ and an $S$-valued point of $\operatorname{Ig}_{\max (a, b)}$ which is neither a cusp nor a supersingular point (in the sense that it corresponds 
to an ordinary elliptic curve with extra structure). If $a \geq b$ and $(E, Q ; \alpha)$ is the given $S$-point of $\operatorname{Ig}_{a}$ then we set $P:=u^{-1} V^{a-b}(Q)$, while if $b \geq a$ and $(E, P ; \alpha)$ is the given $S$-point of $\operatorname{Ig}_{b}$ then we set $Q:=u V^{b-a} P$. Due to [KM, 13.11.3], the data

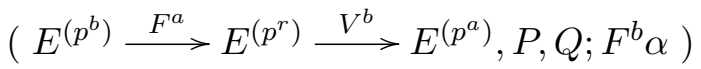

gives an $S$-point of $\mathbf{M}\left(\mathscr{P}_{r}^{1}\right)$. These constructions are visibly inverse to each other.

Remark B.15. When $r$ is even and $a=b=r / 2$, there is a choice to be made as to how one identifies the $(r / 2, r / 2, u)$-component of $\bar{X}_{r}$ with $\operatorname{Ig}_{r / 2}$ : if $\left(\phi: E_{0} \rightarrow E_{r}, P, Q ; \alpha\right)$ is an element of $\mathscr{P}_{r}^{1}\left(E_{0} / S\right)$ which corresponds to a point on the $(r / 2, r / 2, u)$-component, then for $E$ with $E_{0} \simeq E^{\left(p^{r / 2}\right)} \simeq E_{r}$, both $\left(E, P ; V^{r / 2} \alpha p^{-r / 2}\right)$ and $\left(E, Q ; V^{r / 2} \alpha p^{-r / 2}\right)$ are $S$-points of $\operatorname{Ig}_{r / 2}$. Since $u P=Q$, the corresponding closed immersions $\operatorname{Ig}_{r / 2} \hookrightarrow \bar{X}_{r}$ are twists of each other by the automorphism $\langle u\rangle$ of the source. We will consistently choose $\left(E, Q ; V^{r / 2} \alpha p^{-r / 2}\right)$ to identify the $(r / 2, r / 2, u)$-component of $\bar{X}_{r}$ with $\operatorname{Ig}_{r / 2}$.

Remark B.16. As in [MW3, pg. 236], we will refer to $I_{r}^{\infty}:=I_{(r, 0,1)}$ and $I_{r}^{0}:=I_{(0, r, 1)}$ as the two "good" components of $\bar{X}_{r}$. The $\mathbf{Q}_{p}$-rational cusp $\infty$ of $X_{r}$ induces a section of $X_{r} \rightarrow T_{r}$ which meets $I_{r}^{\infty}$, while the section induced by the $K_{r}^{\prime}$-rational cusp 0 meets $I_{r}^{0}$. It is precisely these irreducible components of $\bar{X}_{r}$ which contribute to the "ordinary" part of cohomology. We note that $I_{r}^{\infty}$ corresponds to the image of $\operatorname{Ig}_{r}$ under the map $i_{1}$ of [MW3, pg. 236], and corresponds to the component of $\bar{x}_{r}$ denoted by $C_{\infty}$ in [Tilouine, pg. 343], by $C_{r}^{\infty}$ in [Saby, pg. 231] and, for $r=1$, by $I$ in [Gross, $\S 7$ ].

By base change, the degeneracy mappings (B.6) induce morphisms $\bar{\rho}, \bar{\sigma}: \bar{X}_{r+1} \rightrightarrows \bar{X}_{r}$ of curves over $\mathbf{F}_{p}$ which admit the following descriptions on irreducible components:

Proposition B.17. Let $a, b$ be nonnegative integers with $a+b=r+1$ and $u \in\left(\mathbf{Z} / p^{\min (a, b)} \mathbf{Z}\right)^{\times}$. The restriction of the map $\bar{\sigma}: \bar{X}_{r+1} \rightarrow \bar{X}_{r}$ to the $(a, b, u)$-component of $\bar{X}_{r+1}$ is:

$$
\left\{\begin{array}{lll}
\operatorname{Ig}_{a}=I_{(a, b, u)} \stackrel{F \circ \rho}{\longrightarrow} I_{(a-1, b, u)}=\operatorname{Ig}_{a-1} & : & b<a \leq r+1 \\
\operatorname{Ig}_{b}=I_{(a, b, u)} \stackrel{\langle u\rangle^{-1} F}{\longrightarrow} I_{\left(a-1, b, u \bmod p^{a-1}\right)}=\operatorname{Ig}_{b} & : & a=b=r / 2 \\
\operatorname{Ig}_{b}=I_{(a, b, u)} \stackrel{F}{\longrightarrow} I_{\left(a-1, b, u \bmod p^{a-1}\right)}=\operatorname{Ig}_{b} & : & a<b<r+1 \\
\operatorname{Ig}_{r+1}=I_{(0, r+1,1)} \stackrel{\langle p\rangle_{N} \rho}{\longrightarrow} I_{(0, r, 1)}=\operatorname{Ig}_{r} & : & (a, b, u)=(0, r+1,1)
\end{array}\right.
$$

and the restriction of the map $\bar{\rho}: \bar{X}_{r+1} \rightarrow \bar{X}_{r}$ to the $(a, b, u)$-component of $\bar{X}_{r+1}$ is:

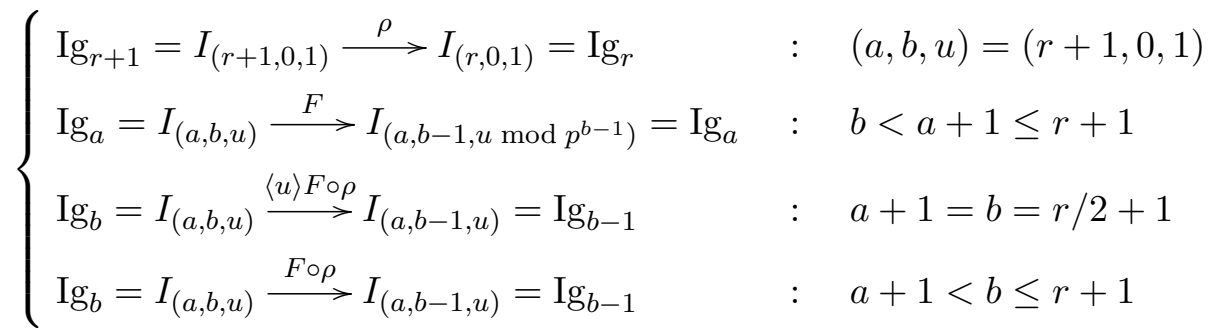

Here, for any $\mathbf{F}_{p}$-scheme $I$, the map $F: I \rightarrow I$ is the absolute Frobenius morphism. 
Proof. Using the moduli-theoretic definitions (B.5) of the degeneracy maps, the proof is a pleasant exercise in tracing through the functorial correspondence between the points of $\bar{X}_{r}$ and points of $\operatorname{Ig}_{(a, b, u)}$. We leave the details to the reader.

We likewise have a description of the automorphism of $\bar{X}_{r}$ induced via base change by the geometric inertia $\operatorname{action}^{16}$ (B.2) of $\Gamma$ :

Proposition B.18. Let $a, b$ be nonnegative integers with $a+b=r$ and $u \in\left(\mathbf{Z} / p^{\min (a, b)} \mathbf{Z}\right)^{\times}$. For $\gamma \in \Gamma$, the restriction of $\bar{\gamma}: \bar{X}_{r} \rightarrow \bar{X}_{r}$ to the $(a, b, u)$-component of $\bar{X}_{r}$ is:

$$
\left\{\begin{array}{l}
\operatorname{Ig}_{a}=I_{(a, b, u)} \stackrel{\mathrm{id}}{\longrightarrow} I_{(a, b, \chi(\gamma) u)}=\operatorname{Ig}_{a} \quad: \quad b \leq a \leq r \\
\operatorname{Ig}_{b}=I_{(a, b, u)} \stackrel{\langle\chi(\gamma)\rangle^{-1}}{\longrightarrow} I_{(a, b, \chi(\gamma) u)}=\operatorname{Ig}_{b} \quad: \quad a<b \leq r
\end{array}\right.
$$

Following [Ulmer, $\S 7-8$ ], we now define a correspondence $\pi_{1}, \pi_{2}: y_{r} \rightrightarrows \mathcal{X}_{r}$ on $\mathcal{X}_{r}$ over $R_{r}$ which naturally extends the correspondence on $X_{r}$ giving the Hecke operator $U_{p}$ (see Appendix A for a brief discussion of correspondences).

Definition B.19. The moduli problem $\mathscr{Q}_{r}^{\zeta}:=\left(\left[\Gamma_{0}\left(p^{r+1}\right) ; r, r\right]^{\zeta \text {-can }} ;\left[\mu_{N}\right]\right)$ on $($ Ell $/ R)$ assigns to $E / S$ the set of quadruples $\left(\phi: E \rightarrow E^{\prime}, P, Q ; \alpha\right)$ where:

(1) $\phi$ is a cyclic $p^{r+1}$-isogeny with standard factorization

$$
E=: E_{0} \stackrel{\phi_{0,1}}{\longrightarrow} E_{1} \cdots \longrightarrow E_{r} \stackrel{\phi_{r, r+1}}{\longrightarrow} E_{r+1}:=E^{\prime}
$$

(2) $P \in E_{1}(S)$ and $Q \in E_{r}(S)$ are generators of $\operatorname{ker} \phi_{1, r+1}$ and $\operatorname{ker} \phi_{r, 0}$, respectively, and satisfy

$$
\left\langle P, \phi_{r, r+1}(Q)\right\rangle_{\phi_{1, r+1}}=\left\langle\phi_{1,0}(P), Q\right\rangle_{\phi_{0, r}}=\zeta \text {. }
$$

(3) $\alpha: \mu_{N} \hookrightarrow E[N]$ is a closed immersion of $S$-group schemes.

Proposition B.20. If $N \geq 4$, then $\mathscr{Q}_{r}^{\zeta}$ is represented by a scheme $\mathbf{M}\left(\mathscr{Q}_{r}^{\zeta}\right)$ that is flat of pure relative dimension 1 over $\operatorname{Spec}(R)$ and regular if $R$ is $R_{r}$-smooth. The compactified moduli scheme $\mathbf{M}^{\mathrm{c}}\left(\mathscr{Q}_{r}^{\zeta}\right)$ is proper and flat of pure relative dimension 1 over $\operatorname{Spec}(R)$, and regular when $R$ is $R_{r}$-smooth.

Proof. Keeping in mind [KM, §7.9], the argument is identical to that of Propositions B.2-B.3.

Definition B.21. We set $y_{r}:=\mathbf{M}^{\mathrm{c}}\left(\mathscr{Q}_{r}^{\varepsilon^{(r)}}\right)$, viewed as a scheme over $T_{r}=\operatorname{Spec}\left(R_{r}\right)$.

Remark B.22. Write $Y_{r}:=X_{1}\left(N p^{r+1} ; N p^{r}\right)$ for the canonical model over $\mathbf{Q}$ with rational cusp $i \infty$ of the modular curve arising as the upper half-plane quotient $\Gamma_{r+1}^{r} \backslash \mathscr{H}^{*}$, for $\Gamma_{r+1}^{r}:=\Gamma_{1}\left(N p^{r}\right) \cap \Gamma_{0}\left(p^{r+1}\right)$. As in Remark B.7, we may identify the generic fiber of $y_{r}$ with the base change $\left(Y_{r}\right)_{K_{r}}$ as follows. First, it is easy to check that $Y_{r}$ is the compactified moduli scheme of quadruples $\left(E_{1}, \iota, \alpha, C\right)$ where $E_{1}$ is an elliptic curve, $\iota: \mu_{p^{r}} \hookrightarrow E_{1}$ and $\alpha: \mu_{N} \hookrightarrow E_{1}$ are embeddings of group schemes, and $C$ is a locally free subgroup scheme of rank $p$ in $E_{1}[p]$ with the property that $C \cap \operatorname{im} \iota=0$. Now given such a quadruple $\left(E_{1}, \iota, \alpha, C\right)$ over a $K_{r}$-scheme $S$, we set $E:=E_{1} / C, E^{\prime}:=E_{1} / \mathrm{im} \iota$, and we denote by $\phi: E \rightarrow E^{\prime}$ the $p^{r+1}$-isogeny

$$
E=E_{1} / C \stackrel{\psi^{t}}{\longrightarrow} E_{1} \stackrel{v}{\longrightarrow} E_{1} / \operatorname{im} \iota=E^{\prime}
$$

\footnotetext{
16 Since $\Gamma$ acts trivially on $\mathbf{F}_{p}$, for each $\gamma \in \Gamma$ the base change of the $R_{r}$-morphism $\gamma: X_{r} \rightarrow\left(\mathcal{X}_{r}\right)_{\gamma}$ along the map induced by the canonical surjection $R_{r} \rightarrow \mathbf{F}_{p}$ is an $\mathbf{F}_{p}$-morphism $\bar{\gamma}: \bar{X}_{r} \rightarrow\left(\bar{X}_{r}\right)_{\gamma} \simeq \bar{X}_{r}$.
} 
where $\psi: E_{1} \rightarrow E_{1} / C$ and $v: E_{1} \rightarrow E_{1} / \mathrm{im} \iota$ are the canonical quotient maps. Using the notation of (B.4), we then put $P_{\iota}:=\iota\left(\varepsilon^{(r)}\right)$ and denote by $Q_{\iota} \in E_{r}(S)$ the unique point satisfying $\left\langle\iota(z), \phi_{r, r+1}\left(Q_{\iota}\right)\right\rangle_{\phi_{1, r+1}}=z$ for all $z \in \mu_{p^{r}}(S)$. The association

$$
\left(E_{1}, \iota, \alpha, C\right) \mapsto\left(\phi: E \rightarrow E^{\prime}, P_{\iota}, Q_{\iota} ; \phi_{1,0} \circ \alpha\right)
$$

then induces the claimed identification.

The scheme $y_{r}$ is equipped with an action of the diamond operators $\mathbf{Z}_{p}^{\times} \times(\mathbf{Z} / N \mathbf{Z})^{\times}$, as well as a "geometric inertia" action of $\Gamma$ given moduli-theoretically exactly as in (B.1) and (B.2).

There is a canonical morphism of curves $\pi: X_{r+1} \rightarrow y_{r}$ over $T_{r+1} \rightarrow T_{r}$ induced by the morphism

$$
\mathscr{P}_{r+1}^{\varepsilon^{(r)}} \rightarrow \mathscr{Q}_{r}^{\varepsilon^{(r)}} \text { given by } \pi\left(\phi: E \rightarrow E^{\prime}, P, Q ; \alpha\right):=\left(\phi: E \rightarrow E^{\prime}, \phi_{0,1}(P), \phi_{r+1, r}(Q) ; \alpha\right) .
$$

It is straightforward to check that the two projection maps $\sigma, \rho: X_{r+1} \rightrightarrows X_{r}$ of (B.5) factor through $\pi$ via unique maps of $T_{r}$-schemes $\pi_{1}, \pi_{2}: y_{r} \rightrightarrows \mathcal{X}_{r}$, given (via Corollary B.5) as morphisms of underlying moduli problems on $\left(\right.$ Ell $\left./ R_{r}\right)$ by

$$
\begin{aligned}
& \pi_{1}\left(\phi: E_{0} \rightarrow E_{r+1}, P, Q ; \alpha\right):=\left(E_{1} \stackrel{\phi_{1, r+1}}{\longrightarrow} E_{r+1}, P, \phi_{r, r+1}(Q) ; \phi_{0,1} \circ \alpha\right) \\
& \pi_{2}\left(\phi: E_{0} \rightarrow E_{r+1}, P, Q ; \alpha\right):=\left(E_{0} \stackrel{\phi_{0, r}}{\longrightarrow} E_{r}, \phi_{1,0}(P), Q ; \alpha\right)
\end{aligned}
$$

That these morphisms are well-defined and that one has $\rho=\pi_{2} \circ \pi$ and $\sigma=\pi_{1} \circ \pi$ are easily verified (see [Ulmer, $\S 7]$ and compare to [KM, $\S 11.3 .3]$ ). They are moreover finite of generic degree $p$, equivariant for the diamond operators, and $\Gamma$-compatible.

Remark B.23. Via the identifications of Remarks B.7 and B.22, on generic fibers the maps $\pi_{1}$ and $\pi_{2}$ are induced by the usual degeneracy maps $\pi_{1}, \pi_{2}: Y_{r} \rightrightarrows X_{r}$ of modular curves over $\mathbf{Q}$ giving the Hecke correspondence $U_{p}:=\left(\pi_{1}, \pi_{2}\right)$. Interpreting $Y_{r}$ and $X_{r}$ moduli-theoretically as in Remarks B.7 and B.22, the map $\pi_{1}$ corresponds to "forget $C$," while $\pi_{2}$ corresponds to "quotient by $C$." We stress that the "standard" degeneracy map $\rho: X_{r+1} \rightarrow X_{r}$ factors through $\pi_{2}$ (and not $\pi_{1}$ ).

Proposition B.24. The scheme $\bar{y}_{r}:=y_{r} \times_{T_{r}} \operatorname{Spec}\left(\mathbf{F}_{p}\right)$ is the disjoint union, with crossings at the supersingular points, of the following proper, smooth $\mathbf{F}_{p}$-curves: for each pair of nonnegative integers $a, b$ with $a+b=r+1$ and for each $u \in\left(\mathbf{Z} / p^{\min (a, b)} \mathbf{Z}\right)^{\times}$, one copy of

$$
\begin{cases}\operatorname{Ig}_{\max (a, b)} & \text { if } a b \neq 0 \\ \operatorname{Ig}_{r} & \text { if }(a, b)=(r+1,0) \text { or }(0, r+1)\end{cases}
$$

We will write $J_{(a, b, u)}$ for the irreducible component of $\bar{y}_{r}$ indexed by $(a, b, u)$, and refer to it as the $(a, b, u)$-component; again, $J_{(a, b, u)}$ is independent of $u$. The proof of Proposition B.24 is a straightforward adaptation of the arguments of [KM, 13.11.2-13.11.4] (see also [Ulmer, Proposition 8.2]). We recall the correspondence between non-cuspidal points of the $(a, b, u)$-component and $\left[\Gamma_{0}\left(p^{r+1}\right) ; r, r\right]^{1-\text { can }_{-}}$ structures on elliptic curves.

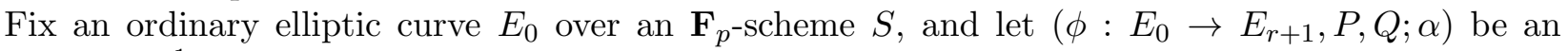
element of $\mathscr{Q}_{r}^{1}\left(E_{0} / S\right)$. As before, there exist unique nonnegative integers $a, b$ with $a+b=r+1$ and a unique elliptic curve $E / S$ with the property that the cyclic $p^{r+1}$-isogeny $\phi$ factors as

$$
E_{0} \simeq E^{\left(p^{b}\right)} \stackrel{F^{a}}{\longrightarrow} E^{\left(p^{r+1}\right)} \stackrel{V^{b}}{\longrightarrow} E^{\left(p^{a}\right)} \simeq E_{r+1}
$$


First suppose that $a b \neq 0$. Then the point $P \in E^{\left(p^{b+1}\right)}(S)$ (respectively $\left.Q \in E^{\left(p^{a+1}\right)}(S)\right)$ is an $\left[\operatorname{Ig}\left(p^{b}\right)\right]$ (respectively $\left[\operatorname{Ig}\left(p^{a}\right)\right]$ ) structure on $E^{(p)}$ over $S$. If $a \geq b$, there is a unit $u \in\left(\mathbf{Z} / p^{b} \mathbf{Z}\right)^{\times}$such that $V^{a-b}(Q)=u P$ in $E^{\left(p^{b+1}\right)}(S)$, while if $a \leq b$ then there is a unique $u \in\left(\mathbf{Z} / p^{a} \mathbf{Z}\right)^{\times}$with $u V^{b-a}(P)=Q$ in $E^{\left(p^{a+1}\right)}(S)$. For $a \geq b$ (respectively $a<b$ ), and fixed $u$, the data $\left(E^{(p)}, Q ; V^{b-1} \alpha p^{1-b}\right.$ ) (respectively $\left(E^{(p)}, P ; V^{b-1} \alpha p^{1-b}\right)$ ) gives an $S$-point of the $(a, b, u)$-component $\operatorname{Ig}_{\max (a, b)}$. If $b=0$ (respectively $a=0$ ), then $Q \in E^{\left(p^{r}\right)}(S)$ (respectively $P \in E^{\left(p^{r}\right)}(S)$ ) is an $\left[\operatorname{Ig}\left(p^{r}\right)\right]$-structure on $E=E_{0}$ (respectively $\left.E=E_{r+1}\right)$. In these extremal cases, the data $(E, Q ; \alpha)$ (respectively $\left.\left(E, P ; V^{r+1} \alpha p^{-r-1}\right)\right)$ gives an $S$-point of the $(r+1,0,1)$-component (respectively $(0, r+1,1)$-component) $\operatorname{Ig}_{r}$.

Conversely, suppose given $(a, b, u)$ and an $S$-point of $\operatorname{Ig}_{\max (a, b)}$ which is neither cuspidal nor supersingular. If $r+1>a \geq b$ and $(E, Q ; \alpha)$ is the given point of $\operatorname{Ig}_{a}$, then we set $P:=u^{-1} V^{a-b}(Q) \in E^{\left(p^{b}\right)}(S)$, while if $r+1>b \geq a$ and $(E, P ; \alpha)$ is the given point of $\operatorname{Ig}_{b}$, we set $Q:=u V^{b-a} P \in E^{\left(p^{a}\right)}(S)$. Then

$$
\left(E^{\left(p^{b-1}\right)} \stackrel{F}{\longrightarrow} E^{\left(p^{b}\right)} \stackrel{F^{a-1}}{\longrightarrow} E^{\left(p^{r}\right)} \stackrel{V^{b-1}}{\longrightarrow} E^{\left(p^{a}\right)} \stackrel{V}{\longrightarrow} E^{\left(p^{a-1}\right)}, P, Q ; F^{b-1} \alpha\right)
$$

is an $S$-point of $\mathbf{M}\left(\mathscr{Q}_{r}^{1}\right)$. If $b=0$ and $(E, Q, \alpha)$ is an $S$-point of $\operatorname{Ig}_{r}$, then we let $P \in E^{(p)}(S)$ be the identity section and we obtain an $S$-point $\left(F^{r+1}: E \rightarrow E^{\left(p^{r+1}\right)}, P, Q ; \alpha\right)$ of $\mathbf{M}\left(\mathscr{Q}_{r}^{1}\right)$. If $a=0$ and $(E, P, \alpha)$ is an $S$-point of $\operatorname{Ig}_{r}$, then we let $Q \in E^{(p)}(S)$ be the identity section and we obtain an $S$-point $\left(V^{r+1}: E^{\left(p^{r+1}\right)} \rightarrow E, P, Q ; F^{r+1} \alpha\right)$ of $\mathbf{M}\left(\mathscr{Q}_{r}^{1}\right)$.

Using the descriptions of $\bar{x}_{r}$ and $\bar{y}_{r}$ furnished by Propositions B.14 and B.24, we can calculate the restrictions of the degenercy maps $\bar{\pi}_{1}, \bar{\pi}_{2}: \bar{y}_{r} \rightrightarrows \bar{x}_{r}$ to each irreducible component of the special fiber of $y_{r}$. The following is due to Ulmer ${ }^{17}$ [Ulmer, Proposition 8.3]:

Proposition B.25. Let $a, b$ be nonnegative integers with $a+b=r+1$ and $u \in\left(\mathbf{Z} / p^{\min (a, b)} \mathbf{Z}\right)^{\times}$. The restriction of the map $\bar{\pi}_{1}: \bar{y}_{r} \rightarrow \bar{X}_{r}$ to the $(a, b, u)$-component of $\bar{y}_{r}$ is:

$$
\left\{\begin{array}{lll}
\operatorname{Ig}_{r}=J_{(r+1,0,1)} \stackrel{F}{\longrightarrow} I_{(r, 0,1)}=\operatorname{Ig}_{r} & : & (a, b, u)=(r+1,0,1) \\
\operatorname{Ig}_{a}=J_{(a, b, u)} \stackrel{\rho}{\longrightarrow} I_{(a-1, b, u)}=\operatorname{Ig}_{a-1} & : & b<a<r+1 \\
\operatorname{Ig}_{b}=J_{(a, b, u)} \stackrel{\left\langle u^{-1}\right\rangle}{\longrightarrow} I_{\left(a-1, b, u \bmod p^{a-1}\right)}=\operatorname{Ig}_{b} & : & a=b=(r+1) / 2 \\
\operatorname{Ig}_{b}=J_{(a, b, u)} \stackrel{\mathrm{id}}{\longrightarrow} I_{\left(a-1, b, u \bmod p^{a-1}\right)}=\operatorname{Ig}_{b} & : & a<b<r+1 \\
\operatorname{Ig}_{r}=J_{(0, r+1,1)} \stackrel{\langle p\rangle_{N}}{\longrightarrow} I_{(0, r, 1)}=\operatorname{Ig}_{r} & : & (a, b, u)=(0, r+1,1)
\end{array}\right.
$$

\footnotetext{
${ }^{17}$ We warn the reader, however, that Ulmer omits the effect of the degeneracy maps on $\left[\mu_{N}\right]$-structures, so his formulae are slightly different from ours.
} 
and the restriction of the map $\bar{\pi}_{2}: \bar{y}_{r} \rightarrow \bar{x}_{r}$ to the $(a, b, u)$-component of $\bar{y}_{r}$ is:

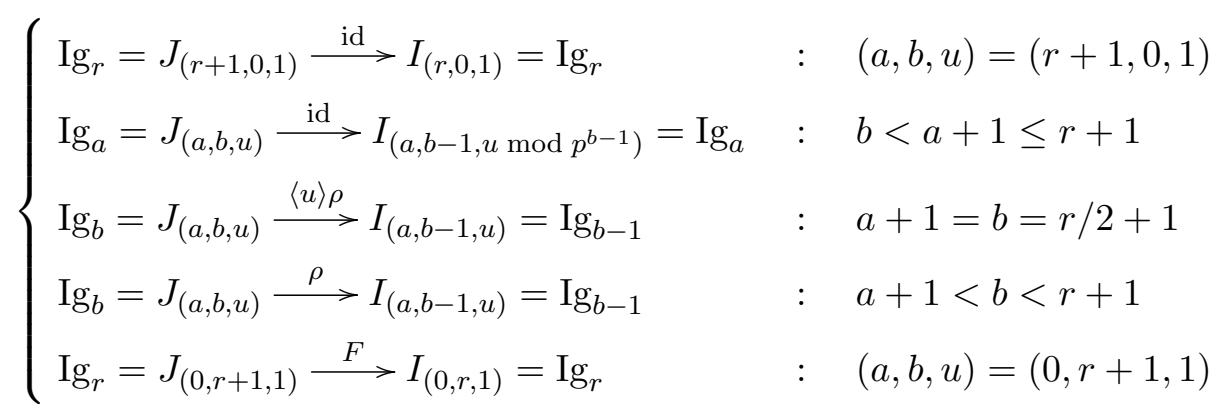

Proof. The proof is similar to the proof of Proposition B.17, using the correspondence between irreducible components of $y_{r}, x_{r}$ and Igusa curves that we have explained, together with the modulitheoretic definitions (B.9) of the degeneracy mappings. We leave the details to the reader.

As we make frequent use of the action of the Hecke operators on the cohomology of $X_{r}$ and $I_{r}$, we would be remiss not to record the definition of the Hecke correspondences on these curves.

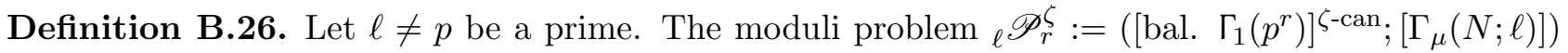
on $($ Ell $/ R)$ assigns to $E / S$ the set of quintuples $\left(\phi: E \rightarrow E^{\prime}, P, Q ; \alpha ; C\right)$ where:

(1) The data $\left(\phi: E \rightarrow E^{\prime}, P, Q ; \alpha\right)$ is an element of $\mathscr{P}_{r}^{\zeta}(E / S)$.

(2) $C \subseteq E[\ell]$ is a locally-free, cyclic, subgroup-scheme of rank $\ell$.

(3) $\operatorname{im}(\alpha) \cap C=0$.

We similarly define the moduli problem $\ell_{\ell} \mathscr{I}_{r}:=\left(\left[\operatorname{Ig}\left(p^{r}\right)\right] ;\left[\Gamma_{\mu}(N, \ell)\right]\right)$ on (Ell $\left./ \mathbf{F}_{p}\right)$ as the functor assigning to $E / S$ the set of quadruples $(E, P ; \alpha ; C)$ where

(1) The triple $(E, R ; \alpha)$ is an element of $\mathscr{I}_{r}(E / S)$.

(2) $C \subseteq E[\ell]$ is a locally-free, cyclic, subgroup-scheme of rank $\ell$.

(3) $\operatorname{im}(\alpha) \cap C=0$

Using the fact that the moduli problem $\left[\mu_{N}\right]$ is representable (recall we assume $N \geq 4$ ), it is not difficult to prove that $\left[\Gamma_{\mu}(N ; \ell)\right]$ is representable as well. Indeed, if $\ell \nmid N$, then $\left[\Gamma_{\mu}(N ; \ell)\right]$ is just the simultaneous moduli problem $\left(\left[\mu_{N}\right],\left[\Gamma_{0}(\ell)\right]\right)$, so representability follows immediately from 3.7 .1 and 4.3.4 of $[\mathrm{KM}]$. When $\ell \mid N$ we instead proceed as follows. Writing $Y_{\mu}(N)$ for the representing object of $\left[\mu_{N}\right]$ and $\left(E^{\text {univ }}, \alpha^{\text {univ }}\right) / Y_{\mu}(N)$ for the universal elliptic curve with universal $\mu_{N}$-structure $\alpha^{\text {univ }}$, the scheme $E^{\text {univ }}[\ell]$ is finite étale over $Y_{\mu}(N)$ since $\ell$ is a unit on the base, and we can form the open subscheme $Z \subseteq E^{\text {univ }}[\ell]$ that is the complement of the closed subscheme $\operatorname{im}\left(\alpha^{\text {univ }}\right)$. It is easy to see that, for any $R$-scheme $S$, the $S$-points of $Z$ parameterize isomorphism classes of triples $(E, \alpha, Q)$ where $\alpha: \mu_{N} \hookrightarrow E[N]$ is a closed immersion of $S$-group schemes and $Q$ is a point of $E[\ell]$ that generates a cyclic subgroup of order $\ell$ which is disjoint from $\operatorname{im}(\alpha)$. There is a natural free action of $(\mathbf{Z} / \ell \mathbf{Z})^{\times}$on $Z$ given by $a:(E, \alpha, Q) \mapsto(E, \alpha, a Q)$, and we write $Z^{\prime}$ for the quotient of $Z$ by this action. Then the $S$-points of $Z^{\prime}$ are identified with isomorphism classes of triples $(E, \alpha, C)$, with $E$ and $\alpha$ as before, and $C$ a locally free, rank $\ell$, cyclic subgroup scheme of $E[\ell]$ with $C \cap \operatorname{im}(\alpha)=0$. Now arguing as in the proof of Proposition B.2, we conclude that $\ell_{\ell_{r}}^{\zeta}$ (respectively $\ell_{\ell} \mathscr{I}_{r}$ ) is represented by a regular (respectively smooth) scheme that is flat of pure relative dimension 1 over $\operatorname{Spec}(R)$. Writing $X_{r}(\ell)$ (respectively $\left.\operatorname{Ig}_{r}(\ell)\right)$ for the corresponding compactified moduli schemes, there are canonical degeneracy mappings 
(keeping in mind Corollary B.5) $\pi_{1}^{(\ell)}, \pi_{2}^{(\ell)}: X_{r}(\ell) \rightrightarrows X_{r}$ given moduli-theoretically by

$$
\begin{aligned}
& \pi_{1}^{(\ell)}\left(\phi: E \rightarrow E^{\prime}, P, Q ; \alpha ; C\right):=\left(\phi: E \rightarrow E^{\prime}, P, Q ; \alpha\right) \\
& \pi_{2}^{(\ell)}\left(\phi: E \rightarrow E^{\prime}, P, Q ; \alpha ; C\right):=\left(\bar{\phi}: E / C \rightarrow E^{\prime} / \phi(C), \psi(P), \psi^{\prime}(Q) ; \psi \circ \alpha\right),
\end{aligned}
$$

where $\psi: E \rightarrow E / C$ and $\psi^{\prime}: E^{\prime} \rightarrow E^{\prime} / \phi(C)$ are the canonical isogenies and $\bar{\phi}$ is the isogeny induced by $\phi$. One similarly defines degeneracy mappings $\pi_{1}^{(\ell)}, \pi_{2}^{(\ell)}: \operatorname{Ig}_{r}(\ell) \rightrightarrows \operatorname{Ig}_{r}$. Writing $T_{\ell}$ (respectively $U_{\ell}$ ) when $\ell \nmid N$ (respectively $\ell \mid N$ ) for the resulting correspondence $\left(\pi_{1}^{(\ell)}, \pi_{2}^{(\ell)}\right.$ ) on $X_{r}$ or $\operatorname{Ig}_{r}$, it is straightforward to check that $T_{\ell}$ and $U_{\ell}$ on $X_{r}$ induce $T_{\ell}$ and $U_{\ell}$, respectively, on the irreducible components $I_{r}^{\infty}=I_{(r, 0,1)}$ and $I_{r}^{0}=I_{(0, r, 1)}$ via Proposition B.14. The correspondence $U_{p}:=\left(\pi_{1}, \pi_{2}\right)$ (respectively $\left.U_{p}^{*}:=\left(\pi_{2}, \pi_{1}\right)\right)$ on $X_{r}$ is defined using the maps (B.9), and induces the correspondence $\left(F\right.$, id) on $I_{r}^{\infty}\left(\right.$ respectively $\left(F,\langle p\rangle_{N}\right)$ on $\left.I_{r}^{0}\right)$.

\section{REFERENCES}

[AEZ] Bernard Angeniol and Fouad El Zein, Appendice: "La classe fondamentale relative d'un cycle", Bull. Soc. Math. France Mém. 58 (1978), 67-93.

[Cais1] Bryden Cais, Canonical integral structures on the de Rham cohomology of curves, Ann. Inst. Fourier (Grenoble) 59 (2009), no. 6, 2255-2300.

[Cais2] Canonical extensions of Néron models of Jacobians, Algebra Number Theory 4 (2010), no. 2, 111-150.

[Cais3] $\quad$ The geometry of Hida families ii: $\lambda$-adic $(\varphi, \gamma)$-modules and $\lambda$-adic Hodge theory, Submitted (2014).

[Cartier] Pierre Cartier, Une nouvelle opération sur les formes différentielles, C. R. Acad. Sci. Paris 244 (1957), 426-428.

[Carayol] Henri Carayol, Sur les représentations l-adiques associées aux formes modulaires de Hilbert, Ann. Sci. École Norm. Sup. (4) 19 (1986), no. 3, 409-468.

[Conrad] Brian Conrad, Grothendieck duality and base change, Lecture Notes in Mathematics, vol. 1750, SpringerVerlag, Berlin, 2000.

[Dee] Jonathan Dee, $\Phi-\Gamma$ modules for families of Galois representations, J. Algebra 235 (2001), no. 2, $636-664$.

[Deligne1] Pierre Deligne, Formes modulaires et representations e-adiques, Séminaire Bourbaki vol. 1968/69 Exposés 347-363 (1971), 139-172.

[Deligne2] _ Théorie de Hodge. II, Inst. Hautes Études Sci. Publ. Math. (1971), no. 40, 5-57.

[EGA] Jean Dieudonné and Alexander Grothendieck, Éléments de géométrie algébrique, Inst. Hautes Études Sci. Publ. Math. (1960-7), no. 4,8,11,17,20,24,28,37.

[DI] Pierre Deligne and Luc Illusie, Relèvements modulo $p^{2}$ et décomposition du complexe de de Rham, Invent. Math. 89 (1987), no. 2, 247-270.

[DS] Pierre Deligne and Jean-Pierre Serre, Formes modulaires de poids 1, Ann. Sci. École Norm. Sup. (4) 7 (1974), 507-530 (1975).

[Edixhoven] Bas Edixhoven, Comparison of integral structures on spaces of modular forms of weight two, and computation of spaces of forms mod 2 of weight one, J. Inst. Math. Jussieu 5 (2006), no. 1, 1-34, With appendix A (in French) by J. F. Mestre and appendix B by Gabor Wiese.

[FK] Takako Fukaya and Kazuya Kato, On conjectures of Sharifi, Preprint (2012).

[FM] Jean-Marc Fontaine and William Messing, p-adic periods and p-adic étale cohomology, Current trends in arithmetical algebraic geometry (Arcata, Calif., 1985), Contemp. Math., vol. 67, Amer. Math. Soc., Providence, RI, 1987, pp. 179-207.

[Gross] Benedict Gross, A tameness criterion for Galois representations associated to modular forms (mod $p$ ), Duke Math. J. 61 (1990), no. 2, 445-517.

[Hartshorne] Robin Hartshorne, Residues and duality, Lecture notes of a seminar on the work of A. Grothendieck, given at Harvard 1963/64. With an appendix by P. Deligne. Lecture Notes in Mathematics, No. 20, SpringerVerlag, Berlin, 1966. 
[Hida1] Haruzo Hida, Galois representations into $\mathrm{GL}_{2}\left(\mathbf{Z}_{p}[[X]]\right)$ attached to ordinary cusp forms, Invent. Math. 85 (1986), no. 3, 545-613.

[Hida2] _ Iwasawa modules attached to congruences of cusp forms, Ann. Sci. École Norm. Sup. (4) 19 (1986), no. 2, 231-273.

[Kitagawa] Koji Kitagawa, On standard p-adic L-functions of families of elliptic cusp forms, p-adic monodromy and the Birch and Swinnerton-Dyer conjecture (Boston, MA, 1991), Contemp. Math., vol. 165, Amer. Math. Soc., Providence, RI, 1994, pp. 81-110.

[KM] Nicholas Katz and Barry Mazur, Arithmetic moduli of elliptic curves, Annals of Mathematics Studies, vol. 108, Princeton University Press, Princeton, NJ, 1985.

[Lazard] Michel Lazard, Commutative formal groups, Lecture Notes in Mathematics, Vol. 443, Springer-Verlag, Berlin, 1975.

[Liu] Qing Liu, Algebraic geometry and arithmetic curves, Oxford Graduate Texts in Mathematics, vol. 6, Oxford University Press, Oxford, 2002, Translated from the French by Reinie Erné, Oxford Science Publications.

[Matsumura] Hideyuki Matsumura, Commutative ring theory, second ed., Cambridge Studies in Advanced Mathematics, vol. 8, Cambridge University Press, Cambridge, 1989, Translated from the Japanese by M. Reid.

[MW1] Barry Mazur and Andrew Wiles, Analogies between function fields and number fields, Amer. J. Math. 105 (1983), no. 2, 507-521.

[MW2] _ Class fields of abelian extensions of Q, Invent. Math. 76 (1984), no. 2, 179-330.

[MW3] _ On p-adic analytic families of Galois representations, Compositio Math. 59 (1986), no. 2, $231-264$.

[Nakajima] Shōichi Nakajima, Equivariant form of the Deuring-Šafarevič formula for Hasse-Witt invariants, Math. Z. 190 (1985), no. 4, 559-566.

[Oda] Tadao Oda, The first de Rham cohomology group and Dieudonné modules, Ann. Sci. École Norm. Sup. (4) $\mathbf{2}(1969), 63-135$.

[Ohta1] Masami Ohta, On the p-adic Eichler-Shimura isomorphism for $\Lambda$-adic cusp forms, J. Reine Angew. Math. 463 (1995), 49-98.

[Ohta2] Ordinary p-adic étale cohomology groups attached to towers of elliptic modular curves. II, Math. Ann. 318 (2000), no. 3, 557-583.

[Raynaud] Michel Raynaud, Géométrie analytique rigide d'après Tate, Kiehl, .., Table Ronde d'Analyse non archimédienne (Paris, 1972), Soc. Math. France, Paris, 1974, pp. 319-327. Bull. Soc. Math. France, Mém. No. 39-40.

[Rosenlicht] Maxwell Rosenlicht, Extensions of vector groups by abelian varieties, Amer. J. Math. 80 (1958), 685-714.

[Saby] Nicolas Saby, Théorie d'Iwasawa géométrique: un théorème de comparaison, J. Number Theory 59 (1996), no. 2, 225-247.

[Sen] Shankar Sen, Continuous cohomology and p-adic Galois representations, Invent. Math. 62 (1980/81), no. 1, 89-116.

[Serre] Jean-Pierre Serre, Sur la topologie des variétés algébriques en caractéristique p, Symposium internacional de topología algebraica International symposium on algebraic topology, Universidad Nacional Autónoma de México and UNESCO, Mexico City, 1958, pp. 24-53.

[SGA6] Théorie des intersections et théorème de Riemann-Roch, Lecture Notes in Mathematics, Vol. 225, SpringerVerlag, Berlin, 1971, Séminaire de Géométrie Algébrique du Bois-Marie 1966-1967 (SGA 6), Dirigé par P. Berthelot, A. Grothendieck et L. Illusie. Avec la collaboration de D. Ferrand, J. P. Jouanolou, O. Jussila, S. Kleiman, M. Raynaud et J. P. Serre.

[Sharifi1] Romyar Sharifi, Iwasawa theory and the eisenstein ideal, Duke Mathematical Journal 137 (2007), no. 1, $63-101$.

[Sharifi2] _ A reciprocity map and the two-variable p-adic L-function, Ann. of Math. (2) 173 (2011), no. 1, 251-300.

[Tate1] John Tate, p-divisible groups., Proc. Conf. Local Fields (Driebergen, 1966), Springer, Berlin, 1967, pp. 158183.

[Tate2] _ Residues of differentials on curves, Ann. Sci. École Norm. Sup. (4) 1 (1968), 149-159.

[Tilouine] Jacques Tilouine, Un sous-groupe $p$-divisible de la jacobienne de $X_{1}\left(N p^{r}\right)$ comme module sur l'algèbre de Hecke, Bull. Soc. Math. France 115 (1987), no. 3, 329-360.

[Ulmer] Douglas Ulmer, On universal elliptic curves over Igusa curves, Invent. Math. 99 (1990), no. 2, $377-391$. 
[Wiles1] Andrew Wiles, On ordinary $\lambda$-adic representations associated to modular forms, Invent. Math. 94 (1988), no. $3,529-573$.

[Wiles2] _ The Iwasawa conjecture for totally real fields, Ann. of Math. (2) 131 (1990), no. 3, 493-540.

University of Arizona, TuCson

Current address: Department of Mathematics, 617 N. Santa Rita Ave., Tucson AZ. 85721

E-mail address: cais@math.arizona.edu 